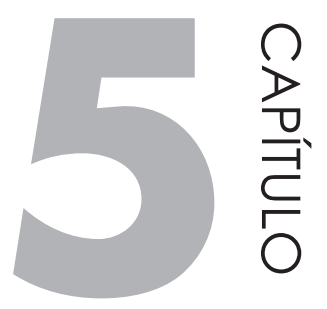

\title{
INTERAÇÃO ELÉTRICA E MAGNÉTICA
}

\subsection{LEI DE COULOMB}

Em 1785, Coulomb (1736-1806) mediu o valor das forças elétricas de atração e repulsão, obtendo a lei que as descreve. Usou uma balança de torção parecida com a de Cavendish, posteriormente utilizada na medida da atração gravitacional.

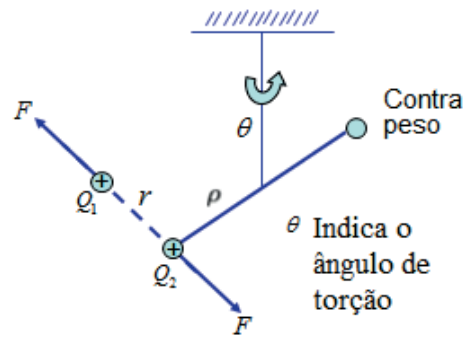


Em seguida, obteve uma equação semelhante à de Newton para a interação gravitacional:

$$
\begin{gathered}
F= \pm \frac{1}{4 \pi \varepsilon_{0}} \frac{Q_{1} Q_{2}}{r^{2}} \\
\varepsilon_{0}=8,85418 \times 10^{-12} C^{2} / \mathrm{Nm}^{2}{ }^{1}
\end{gathered}
$$

C = Coulomb, unidade de carga elétrica

$$
Q_{1} \text { e } Q_{2} \text { cargas elétricas }
$$

A força entre duas cargas $Q_{1}$ e $Q_{2}$ é diretamente proporcional às cargas elétricas e inversamente proporcional ao quadrado da distância $r$ entre as duas. Quando $Q_{1}$ e $Q_{2}$ têm mesmo sinal, haverá repulsão; quando têm sinais contrários, atração.

Calibra-se o aparelho com uma força conhecida (p. ex., um peso) que causará uma deflexão $\theta$. As outras forças ocasionarão deflexões proporcionais. Variando-se as cargas e a distância entre elas, obtemos forças diferentes. Adiante, veremos um exemplo de como avaliar a viabilidade.

Dessa forma:

\begin{tabular}{|l|l|l|l|}
\hline Distâncias, ângulo de torção & $36^{\circ}$ & $18^{\circ}$ & $9^{\circ}$ \\
\hline Forças, ângulo de torção & $36^{\circ}$ & $144^{\circ}=36^{\circ} \times 4$ & $576^{\circ}=36^{\circ} \times 16$ \\
\hline
\end{tabular}

Quando a distância se torna duas, depois quatro vezes menor, a força se torna quatro e, em seguida, 16 vezes maior, o que verifica a lei, pois as forças são inversamente proporcionais aos quadrados dos ângulos de torção e, portanto, aos quadrados das distâncias. ${ }^{2}$

Sendo $\theta$ o ângulo, $C \theta$ o momento proporcional ao ângulo de torção $\theta, C \theta$ o par de torção, $C$ constante de torção que depende do comprimento $\ell$, diâmetro $d$ e da natureza do fio.

Coulomb estabeleceu a lei da torção: $\quad C=k \frac{d^{4}}{\ell}$

1 Constante dielétrica ou de permissividade, ver Seção 5.10. Fazendo: $F=K_{e} Q_{1} Q_{2} / r^{2}$. Atribuindo a $K_{e}$ um valor conveniente: $K_{e}=10^{-7} c^{2}=8,9874 \times 10^{9}, \quad c=$ velocidade da luz no vácuo. $\therefore K_{e} \cong 9 \times 10^{9}$. Logo $K_{e}=1 / 4 \pi \varepsilon_{0}$ e resulta $\varepsilon_{0}=8,85418 \times 10^{-12} C^{2} / N^{2}$. Definição de Coulomb: é a carga que, quando colocada no vácuo, a um metro de uma carga igual, repele-a com uma força de $8,9874 \times 10^{9} \mathrm{~N}$. A conveniência desse valor é que $c^{2}=1 / \varepsilon_{0} \mu_{0}$, sendo $\mu_{0}$ a constante de permeabilidade, como será visto nas Seções 5.16, 5.23 e 5.24.

2 O ângulo $\theta$ é proporcional ao arco $\theta \rho$, onde $\rho=$ raio (distância ao centro de rotação), logo $\theta \rho$ é uma distância (ver figura da Seção 5.1, página inicial). 
$k$ é o coeficiente que depende da natureza do fio (ver Capítulo 3, "Elasticidade"; ALONSO, FINN, LEMOINE, GUYOT).

Leis como a da interação gravitacional e a de Coulomb, que variam com o inverso do quadrado da distância, são denominadas de leis do inverso do quadrado (RAINICH).

Se igualarmos as forças gravitacionais e elétricas: $\mathrm{G} \frac{\mathrm{m}_{1} \mathrm{~m}_{2}}{\mathrm{r}^{2}}=\mathrm{K}_{\mathrm{e}} \frac{\mathrm{Q}_{1} \mathrm{Q}_{2}}{\mathrm{r}^{2}}(1)$

E multiplicarmos essas forças por $\mathrm{r}$, obtemos o trabalho $\mathrm{W}=\mathrm{F} \cdot \mathrm{r}$, que é uma forma de energia.

Usando unidades gaussianas cgs, sabemos que $\mathrm{K}_{\mathrm{e}}=10^{-7} \mathrm{c}^{2}$ (ver Seção 5.1). ${ }^{3}$

Valor de $\mathrm{K}_{\mathrm{e}}=1 \mathrm{~d} . \mathrm{cm}^{2} /$ statc $^{2}$ [ver observação na subseção 5.1.2 Equação (1)]

Valor de $\mathrm{G}=6,67 \times 10^{-11} \mathrm{~N} \cdot \mathrm{m}^{2} / \mathrm{kg}^{2}$, em unidades cgs:

Referência: LANDAU

$\mathrm{G}=6,67 \times 10^{-8} \mathrm{~d} \cdot \mathrm{cm}^{2} / \mathrm{g}^{2}$

Há, portanto, uma semelhança formal entre as duas formas de energia. Isso não é fortuito, pois como veremos na Seção 7.7, Equação (1), existe uma relação entre massa e energia, como deduzido na famosa fórmula de Einstein: $E_{k}=c^{2} \Delta m$, em que a velocidade da luz no vácuo, $c$, tem um importante significado.

Isso explica porque o deficit de massa ${ }^{4}$ resultante das reações atômicas se converte em energia, que é aproveitada nos reatores nucleares e nas bombas atômicas. Também explica de onde as estrelas obtêm energia, convertendo $\mathrm{H}$ em He, e, como explicado pelo ciclo de Bethe, as estrelas geram os elementos leves: $\mathrm{C}, \mathrm{N}$ e $\mathrm{O}$ (GAMOW).

De modo semelhante, as estrelas geram os elementos até o Fe. E os mais pesados que o Fe são gerados quando as estrelas morrem nas explosões estelares denominadas novas e supernovas, tão espetaculares que sua luminosidade supera a de uma galáxia inteira. Concluímos então, que "somos formados do pó das estrelas extintas".

Observando-se a Equação (1), deduzimos haver uma relação entre carga e massa Q/m. De fato, é possível verificar pelas experiências realizadas por J. J. Thomson, em 1897, no Exemplo ilustrativo da Seção 7.4, sobre a descoberta do elétron, em que ele mediu a relação entre a carga do elétron e e sua massa $\mathrm{m}: \mathrm{e} / \mathrm{m}$. Podemos afirmar que a carga do elétron é realmente o quantum das cargas elétricas, isto é, a menor carga elétrica possível, e toda carga elétrica é um múltiplo dessa carga.

$\mathrm{K}_{\mathrm{e}}=10^{-7} \mathrm{c}^{2}=8,9874 \times 10^{9}$, em unidades MKS, em unidades cgs, o valor dado a seguir: $\mathrm{K}_{\mathrm{e}}=1 \mathrm{~d} . \mathrm{cm}^{2} /$ statc .

${ }^{4}$ Isto é, o que falta no resultado final com relação às massas iniciais. Observe que $\Delta \mathrm{m}$ é a variação da massa, ou seja, a massa faltante. Podemos, então, pensar que a massa seria uma forma de energia condensada e, consequentemente, a massa faltante $\Delta \mathrm{m}$ seria liberada como energia cinética $\mathrm{E}_{\mathrm{k}}$ durante a reação nuclear. 
Thomson mediu: $\mathrm{e} / \mathrm{m}=1,7 \times 10^{11 \mathrm{C}} / \mathrm{kg}$, em excelente concordância com o valor atual: $1,75890 \times 10^{11} \mathrm{C} / \mathrm{kg}$, ou, no sistema cgs, Thomson teria encontrado: $\mathrm{e} / \mathrm{m}=5,1 \times 10^{17} \mathrm{statc} / \mathrm{g}$, pois $1 \mathrm{C}=$ $2,99592 \times 10^{9}$ statc $\cong 3 \times 10^{9}$ statc.

A experiência de J.J. Thomson foi realizada com tubo de raios catódicos, conforme apresentado na Seção 7.4, Exemplo ilustrativo, sobre a descoberta do elétron.

O valor obtido é cerca de 1.800 vezes maior que o valor conseguido com íons de $\mathrm{H}$ na eletrólise (com maior exatidão 1836,15; HALLIDAY, RESNICK, WALKER). Ver final da Seção 7.5, sobre as experiências levadas a efeito por Faraday em 1833:

$$
\mathrm{e} / \mathrm{m}_{\mathrm{H}}=95721 \mathrm{C} / \mathrm{atg}=2,8716 \times 10^{14} \text { statc/g (deve-se levar em conta que } 1 \text { atg } \mathrm{H}=1,008 \mathrm{~g} \text { ). }
$$

Como o valor encontrado por Thomson era independente do material usado no cátodo e do gás usado no tubo de raios catódicos, ${ }^{5}$ ele assumiu que as partículas dos raios catódicos teriam massa cerca de 1.800 vezes menor que a do íon $H$, então supôs que a massa da partícula seria cerca de $1 / 1800$ a do íon H. Considerou que as partículas fossem uma nova espécie de "corpúsculo negativo", o qual, por sugestão de Stoney em 1874 (ver Anexo 11, "Da importância de alguém que andou meio esquecido"), chamamos hoje de elétron. Stoney previra sua existência como a "unidade natural de eletricidade", ou seja, a quantidade de eletricidade que deve passar através da solução a fim de liberar, em um dos eletrodos, um átomo de $\mathrm{H}$ ou um átomo de qualquer substância univalente.

Em 1873, Maxwell, investigando a determinação da velocidade da luz no vácuo, em função da constante eletrostática e eletrodinâmica: $c=\frac{1}{\sqrt{\varepsilon_{0} \mu_{0}}}$ [Seção 5.23, Equação (5)], previu a existência das ondas eletromagnéticas com as equações: $\frac{\partial^{2} \mathrm{E}}{\partial \mathrm{t}^{2}}=\frac{1}{\varepsilon_{0} \mu_{0}} \frac{\partial^{2} \mathrm{E}}{\partial \mathrm{x}^{2}} \mathrm{e} \frac{\partial^{2} \mathrm{~B}}{\partial \mathrm{t}^{2}}=\frac{1}{\varepsilon_{0} \mu_{0}} \frac{\partial^{2} \mathrm{~B}}{\partial \mathrm{x}^{2}}$ [Seção 5.23, Equações (4) e (6)], a partir da qual se deduz que $\mathrm{v}=\mathrm{c}=\frac{1}{\sqrt{\varepsilon_{0} \mu_{0}}}$, confirmando a equação apresentada, por sua vez, deduzida por Kohlrausch e Weber em 1832.

Hertz, em 1888, verificou a existência das ondas eletromagnéticas com seu oscilador.

O valor de $\varepsilon_{0}$ foi estabelecido como: $\varepsilon_{0}=\frac{10^{7}}{4 \pi \mathrm{c}^{2}}=8,854 \times 10^{-12} \mathrm{C}^{2} /\left(\mathrm{N} . \mathrm{m}^{2}\right)$

Podemos verificar que a força elétrica se torna $\mathrm{F}=\frac{1}{4 \pi \varepsilon_{0}} \frac{\mathrm{QQ} \mathrm{r}^{\prime}}{\mathrm{r}^{2}}$, e, substituindo pelo valor acima, temos: $\mathrm{F}=\frac{1}{4 \pi} \frac{4 \pi \mathrm{c}^{2}}{10^{7}} \frac{\mathrm{QQ}^{\prime}}{\mathrm{r}^{2}}$, como: $\mathrm{F}=\mathrm{K}_{\mathrm{e}} \frac{\mathrm{QQ}^{\prime}}{\mathrm{r}^{2}}$, obtemos: $\mathrm{K}_{\mathrm{e}}=10^{-7} \mathrm{c}^{2} \mathrm{~N} \cdot \mathrm{m}^{2} / \mathrm{C}^{2}$ em unidades MKS, como visto inicialmente, portanto, em função da velocidade da luz no vácuo c.

Posteriormente, Bucherer, em 1909, testou a relatividade da massa medindo e/m em função de v, conforme relatado no início da Seção 7.5 .

Levando em consideração que $\mathrm{v}=\mathrm{E} / \mathrm{B},{ }^{6}$ obteve:

Materiais usados no cátodo: Al, Fe e Pt. Gases usados: $\mathrm{Ar}, \mathrm{H}_{2}$ e $\mathrm{CO}_{2}$.

Em que v é a velocidade dos elétrons no feixe eletrônico da experiência de Thomson no tubo de raios catódicos. 
$\frac{\mathrm{e}}{\mathrm{m}}=\frac{\mathrm{e}}{\mathrm{m}_{0}}\left(1-\frac{\mathrm{v}^{2}}{\mathrm{c}^{2}}\right)^{1 / 2}$, ver a relação $\mathrm{Q} / \mathrm{m}_{0}$, a seguir.

Da relatividade, sabemos que a massa de repouso $\mathrm{m}_{0}$, a carga e e a velocidade c são constantes, logo, a relação $\mathrm{e} / \mathrm{m}$ e, portanto $\mathrm{Q} / \mathrm{m}$, pois $\mathrm{Q}$ é múltiplo de e, é função da velocidade da luz no vácuo $\mathrm{c}$, justificando a utilização de $\mathrm{K}_{\mathrm{e}}=10^{-7} \mathrm{c}^{2}$, como visto no início (KAPLAN, SEARS, HALLIDAY, RESNICK).

Para generalizar, aplica-se a fórmula da força eletromagnética de Lorentz: $\vec{F}=Q(\vec{E}+\vec{v} \times \vec{B})$, como visto no final da Seção 7.4, obtendo: $\frac{\mathrm{Q}}{\mathrm{m}_{0}}=\frac{1}{\overrightarrow{\mathrm{E}}+\overrightarrow{\mathrm{v}} \times \overrightarrow{\mathrm{B}}}\left[\frac{\mathrm{d}}{\mathrm{dt}}(\gamma \overrightarrow{\mathrm{v}})\right]$, em que: $\gamma=\frac{1}{\sqrt{1-\beta^{2}}}$ e $\beta=\frac{\mathrm{v}}{\mathrm{c}}$.

Mais uma vez, observamos que c desempenha seu papel.

A seguir, será relatado como as ideias evoluíram.

Expressando a lei de Coulomb como $F=\frac{1}{4 \pi \varepsilon_{0}} \frac{Q_{1} Q_{2}}{r^{2}}, 7$ no sistema de unidades MKSC (Seção 5.1), em que $4 \pi$ aparece considerando a carga $Q_{1}$ no centro de um espaço em que sua influência é exercida sobre a carga $Q_{2}$, situada a uma distancia $r$ sobre uma superfície esférica com ângulo sólido total de $4 \pi$ esferorradianos [Seção 5.10, Equação (1)], o índice 0 da constante dielétrica $\varepsilon_{0}$ indica seu valor no vácuo (Seção 5.1).

Até então, trabalhamos somente com cargas em repouso, ao que damos o nome de Eletrostática.

Ao pesquisar a corrente em condutores, denominada Eletrodinâmica, verificou-se que ela causa um campo magnético em torno do condutor. Esse campo foi definido experimentalmente como: $B=\frac{\mu_{0}}{2 \pi r} \mathrm{i}$. Veremos na Seção 5.16, Equação (1), que a circulação magnética resulta em:

$$
\Gamma=\oint_{C} \vec{B} \cdot d \vec{\ell}=\mu_{0} \mathrm{i}
$$

Chamada de lei de Ampère, originando a constante de permeabilidade $\mu_{0}$ (ver início da Seção 5.16 e final da Seção 5.23, sobre a velocidade da luz no vácuo).

Por outro lado, Faraday, em 1831, chegou à lei da indução: $\oint \overrightarrow{\mathrm{E}} \cdot \mathrm{dl}=\frac{\mathrm{d} \phi_{\mathrm{B}}}{\mathrm{dt}}$, ou seja, um campo magnético variável, com o tempo, produz um campo elétrico (i.e., uma corrente) em um condutor (final da Seção 5.14).

Em 1832, Kohlrausch e Weber determinaram a relação da constante eletrostática e eletrodinâmica, encontrando: $\mathrm{c}=\frac{1}{\sqrt{\varepsilon_{0} \times \mu_{0}}}=\frac{1}{\sqrt{\left(4 \pi \times 10^{-7}\right)\left(8,9 \times 10^{-12}\right)}}=3 \times 10^{8} \mathrm{~m} / \mathrm{s}$, isto é, a velocidade da luz no vácuo.

Medida pela primeira vez em 1785 por Coulomb. Mostrou que seguia uma lei da forma: $\mathrm{F} \propto \frac{1}{\mathrm{r}^{2}}$, em que $\propto$ significa proporcional. Na época, não havia um conceito preciso sobre carga. Trabalhos posteriores indicaram a influência das cargas: $\mathrm{F} \propto \frac{\mathrm{Q}_{1} \mathrm{Q}_{2}}{\mathrm{r}^{2}} \mathrm{e}$, finalmente, chegou-se à equação mencionada. 
Maxwell, tomando conhecimento dessa relação, investigou teoricamente, resultando em seu Tratado de Eletricidade e Magnetismo, publicado em 1873.

Considerando que a luz se propagando no vácuo, isto é, sem cargas livres nem corrente, ele deduziu as equações:

Lei de Faraday-Henry $\frac{\partial \mathrm{E}}{\partial \mathrm{x}}=-\frac{\partial \mathrm{B}}{\partial \mathrm{t}}$

e a lei de Ampère-Maxwell $-\frac{\partial \mathrm{B}}{\partial \mathrm{x}}=\varepsilon_{0} \mu_{0} \frac{\partial \mathrm{E}}{\partial \mathrm{t}}$

Ver Seção 5.23, Equações (1) e (3).

Derivando (2) em relação a $\mathrm{x}$ e (3) em relação a $\mathrm{t}$, obtemos: $\frac{\partial^{2} \mathrm{E}}{\partial \mathrm{x}^{2}}=-\frac{\partial^{2} \mathrm{~B}}{\partial \mathrm{x} \partial \mathrm{t}} \mathrm{e}-\frac{\partial^{2} \mathrm{~B}}{\partial \mathrm{t} \partial \mathrm{x}}=\varepsilon_{0} \mu_{0} \frac{\partial^{2} \mathrm{E}}{\partial \mathrm{t}^{2}}$. Combinando as duas, teremos: $\frac{\partial^{2} \mathrm{E}}{\partial \mathrm{t}^{2}}=\frac{1}{\varepsilon_{0} \mu_{0}} \frac{\partial^{2} \mathrm{E}}{\partial \mathrm{x}^{2}}$, precisamente a equação diferencial de segunda ordem de uma onda: $\frac{\partial^{2} \xi}{\partial \mathrm{t}^{2}}=\mathrm{v}^{2} \frac{\partial^{2} \xi}{\partial \mathrm{x}^{2}}$.

[Seção 5.23, Equação (2); ver também Anexo 6, Equações (0) e (1), Resolução da Equação da Onda de Schrödinger, e em outras palavras].

Por onde se deduz que a velocidade: $\mathrm{v}=\frac{1}{\sqrt{\varepsilon_{0} \times \mu_{0}}}=\mathrm{c}$, confirmando a equação obtida por Kohlrausch e Weber, como já mencionado.

De modo semelhante também se obtém: $\frac{\partial^{2} \mathrm{~B}}{\partial \mathrm{t}^{2}}=\frac{1}{\varepsilon_{0} \mu_{0}} \frac{\partial^{2} \mathrm{~B}}{\partial \mathrm{x}^{2}}$

Maxwell concluiu que a luz são ondas elétricas e magnéticas se propagando no vácuo na velocidade da luz c. Previu, então, a existência das ondas eletromagnéticas [Seção 5.23, Equações (4), (5), (6) e (7)], cuja existência seria confirmada por Hertz em 1888, com seu oscilador. Por isso, essas ondas ficaram conhecidas como ondas hertzianas. A partir de então, a ótica se tornou parte do estudo do eletromagnetismo. Posteriormente, descobriram-se novos campos para essas ondas, com frequências diferentes da luz: infravermelho, ultravioleta, raios $\mathrm{X}$, ondas de rádio etc. $\mathrm{O}$ raio $\mathrm{X}$ comprovou ser eficiente no diagnóstico médico, e as ondas de rádio, na telecomunicação telegráfica, radiocomunicação, TV etc. Vale mencionar a descoberta posterior da radiação $\gamma$, resultante da desintegração atômica (ALONSO, FINN). ${ }^{8}$

O problema da radiação térmica foi resolvido por Planck em 1901, com uma hipótese revolucionária. Ele postulou que a energia da radiação, em vez de utilizar valores de zero ao infinito, tomam valores de 0 a $n \varepsilon_{0}$, sendo $n$ valores inteiros e $\varepsilon_{0}$ o denominado quantum, e no plural, quanta, daí o conceito de "quantização da energia". ' Porém, ainda se considerava, conforme Maxwell, a radiação térmica, parte das oscilações eletromagnéticas, como um fenômeno ondulatório.

\footnotetext{
Também vale ressaltar o surgimento de um campo novo: a Radioastronomia.

Trata-se do início da Mecânica Quântica, embora Planck não tivesse consciência disso.
} 
Somente em 1905, Einstein, ao publicar três artigos, ${ }^{10}$ um deles sobre o efeito fotoelétrico, aplicou o conceito de Planck para explicar como a radiação ultravioleta, ao incidir sobre uma placa metálica, libera elétrons que podem ser detectados na forma de corrente elétrica. Ele explicou que a radiação é formada de partículas, os quanta da energia, denominando-as de "fótons".

Isso seria confirmado por Compton, em sua experiência denominada efeito Compton, de 1928, na qual demonstrou que os fótons de raios X incidem sobre elétrons livres e, assim como bolas de bilhar, provocam a transferência da quantidade de movimento, deslocando os elétrons e sendo desviados por eles, como partículas.

Einstein chegou à conclusão que a radiação eletromagnética tem natureza dualística, comportandose como onda sob certas circunstâncias, e como partículas ou fótons sob outras.

Todavia, em 1905, a existência do átomo ainda era posta em dúvida (MACH). Em 1916, Einstein publica artigo sobre a Relatividade geral e, em 1921, recebe o Prêmio Nobel pela explicação do efeito fotoelétrico. Contudo, as ideias sobre Relatividade demorariam a ser aceitas e somente o foram graças aos esforços de Sir Arthur Eddington, Schwarschild (curvatura da luz sob efeito gravitacional e buracos negros), Otto Hahn e Lise Meitner (em 1938, fissão do Urânio), Oppenheimer e muitos outros.

\subsubsection{Exemplo ilustrativo}

Para avaliar melhor a viabilidade da utilização da balança de torção, vejamos um exemplo:

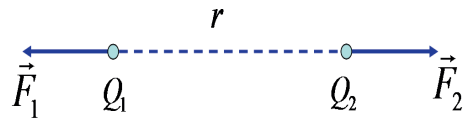

Sejam duas cargas: $Q_{1}=-1,0 \times 10^{-6} \mathrm{C}$ e $Q_{2}=-3,0 \times 10^{-6} \mathrm{C}$, distantes entre si de $r=15 \mathrm{~cm}$.

Qual é a força que atua nas cargas $Q_{1}$ e $Q_{2}$ ?

Como as cargas têm sinal igual, as forças $F_{1}$ e $F_{2}$ serão de repulsão:

$$
\begin{gathered}
F_{1}=-F_{2}=\frac{1}{4 \pi \varepsilon_{0}} \frac{Q_{1} Q_{2}}{r^{2}}=\frac{\left(8,9874 \times 10^{9} \mathrm{Nm}^{2} / \mathrm{C}\right)\left(1,0 \times 10^{-6} \mathrm{C}\right)\left(3,0 \times 10^{-6} \mathrm{C}\right)}{\left(1,5 \times 10^{-1} \mathrm{~m}\right)^{2}}= \\
=1,198 \mathrm{~N}=\frac{1,198 \mathrm{~N}}{9,81^{\mathrm{N}} / \mathrm{kg} f}=0,122 \mathrm{kgf}=122 \mathrm{gf}
\end{gathered}
$$

Resolvendo no sistema gaussiano:

${ }^{10}$ Os outros dois eram: "Movimento browniano", em que se comprovava a existência do átomo, e "Teoria da relatividade especial ou restrita", nos Annalen der Physick. 


$$
\begin{gathered}
Q_{1}=-1,0 \times 10^{-6} \mathrm{C}=-1,0 \times 10^{-6} \times 2,99592 \times 10^{9} \text { statcoulomb }=-2,99592 \times 10^{3} \text { statc } \\
Q_{2}=-3,0 \times 10^{-6} C \times 2,99592=-8,98776 \text { statc } \\
F_{1}=-F_{2}=\frac{\left(2,99592 \times 10^{3} \text { statc }\right)\left(8,98776 \times 10^{3} \text { statc }\right)}{(1,5 \times 10 \mathrm{~cm})^{2}}=11,96738 \times 10^{4}=119673,8 \text { dinas }= \\
=\frac{119673,8}{981}=122 \mathrm{gf}
\end{gathered}
$$

É possível trabalhar com cargas da ordem de statc, ou unidades eletrostáticas, ues, distâncias em cm, e forças da ordem de centenas de $g f$. As cargas em Coulomb $C$ seriam grandes demais.

\subsubsection{Observação}

Pode-se observar que ao usar o sistema gaussiano, na fórmula da lei de Coulomb:

$$
F=K_{e} \frac{Q_{1} Q_{2}}{r^{2}} \quad K_{e}=1 d \cdot \mathrm{cm}^{2} / \text { statc }^{2}
$$

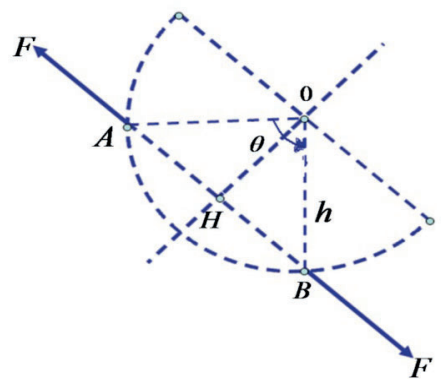

$O H=h \cos \frac{\theta}{2}$, momento em relação ao eixo de rotação de força elétrica $F$ :

$F \times O H=F h \cos \frac{\theta}{2}$, equação de equilíbrio:

$F h \cos \frac{\theta}{2}=C \theta \therefore F=\frac{C \theta}{h \cos \frac{\theta}{2}},{ }^{11}$ por exemplo:

${ }^{11}$ Ver Capítulo 3, "Elasticidade", sobre o momento de torção ou conjugada, $\mathrm{C}=\mathrm{k} \frac{\mathrm{d}^{4}}{\ell}$, $\mathrm{k}$ nas mesmas unidades que o módulo de rigidez $\mathrm{G}, \mathrm{kgf} / \mathrm{cm}^{2}$. 
Suponha $\theta=1 \mathrm{rad}$ e $h=15,64 \mathrm{~cm}$

$\cos 0,5 \mathrm{rad}=0,87758$, comprimento do fio de torção $\ell=50 \mathrm{~cm}$, diâmetro do fio

$$
\mathrm{d}=2,17 \mathrm{~mm}=2,17 \times 10^{-1} \mathrm{~cm} .
$$

Então: $F=k \frac{d^{4}}{\ell} \frac{\theta}{h \cos \frac{\theta}{2}}$. Para fio de prata, tem-se em unidades gaussianas $k=2,7 \times 10^{10}, \mathrm{kgf} / \mathrm{cm}^{2}$,

$$
\begin{aligned}
\mathrm{OH}=\mathrm{h} \cos \theta / 2=11,395 \times 0,87758=10 \mathrm{~cm} & \\
\therefore F=2,7 \times 10^{10} \times \frac{2,17 \times 10^{-4}}{50} \times \frac{1}{10}=119.674 \text { dinas } \quad & \text { AB }=2 \text { hsen } \theta / 2=15 \mathrm{~cm} \\
& \operatorname{sen} \theta / 2=0,47943
\end{aligned}
$$

\subsection{FLUXO DE UM LÍQUIDO}

A noção de fluxo deriva do escoamento de um líquido pela secção de um tubo ou canal.

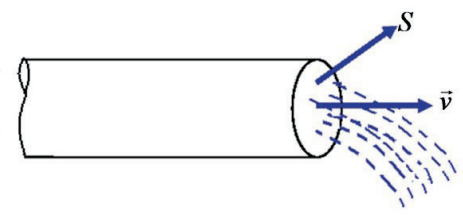

Supondo a velocidade $\vec{v}$ constante na secção $S$, a vazão, ou fluxo, do líquido será:

$$
\phi=v S \mathrm{~cm} / \mathrm{s} \times \mathrm{cm}^{2} \Rightarrow \mathrm{cm}^{3} / \mathrm{s} .
$$

$\mathrm{Na}$ prática, todo líquido é viscoso, o que provoca atrito interno no escoamento. As partículas que fluem por $S$ não têm a mesma velocidade, sendo nulas nas margens e aumentando até atingir um máximo no eixo da tubulação.

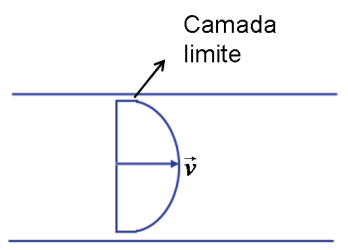

Como $\vec{v}$ varia na secção, teremos um fluxo elementar: $d \phi=v d S$, em um elemento de área dS da área total S. 
Integrando por toda secção: $\phi=\iint_{S} v d S$

Referência: Maurer, J. M. Azevedo Netto

\subsection{GRANDEZAS ESCALARES, VETORIAIS E TENSORIAIS: CAMPOS}

Grandezas escalares são as que necessitam somente da especificação da magnitude e do sinal, e podem ser positivas, negativas ou nulas. Exemplos: temperatura, volume, massa, carga elétrica etc. Além da magnitude, as grandezas vetoriais necessitam da especificação direcional, por exemplo, velocidade, momento, força etc.

O tensor é uma generalização dos conceitos de escalar e vetor. Dessa forma, eles são casos especiais. O escalar é um tensor de ordem zero, e o vetor, de primeira ordem. O tensor é uma entidade que, com a mudança do sistema de coordenadas (por translação e/ou rotação), sofre mudança de suas componentes, porém, a grandeza em si é invariante.

Um tensor de segunda ordem também é chamado de diádica. Exemplos de tensor de segunda ordem são a tensão e a deformação. Um tensor de terceira ordem é chamado de triádica. Se for de ordem $n$, será uma poliádica. As leis físicas, para serem válidas, devem ser independentes do sistema de coordenadas para exprimi-las matematicamente.

Conforme a grandeza considerada, podemos expressá-la em um espaço tridimensional (x, y, z), tetradimensional $(\mathrm{x}, \mathrm{y}, \mathrm{z}, \mathrm{t})$ ou, generalizando, $m$ dimensional.

Um “campo" é uma distribuição contínua de quantidades escalares, vetoriais ou tensoriais, descritas por funções contínuas de coordenadas espaciais e do tempo. Por exemplo, a temperatura em todos os pontos de um corpo, em qualquer instante, pode ser descrita pelo campo escalar $T$ (x, y, z, t).

Um campo vetorial, como o da velocidade, pode ser designado por $\vec{v}$ (x, y, z, t). Isso significa que $\vec{v}=f(x, y, z, t)$, é uma função das variáveis $\mathrm{x}, \mathrm{y}, \mathrm{z}, \mathrm{t}$. Na verdade cada uma de suas componentes são funções de cada uma das coordenadas, respectivamente, $x, y, z$ e t. Uma diádica tridimensional tem $3^{2}=$ 9 componentes, e uma diádica tetradimensional, $4^{2}=16$ componentes.

\section{Observação}

Embora o quociente entre dois vetores não possa ser definido satisfatoriamente, os tensores surgem fisicamente em circunstâncias que os fazem parecer com uma divisão. Por exemplo, tensão é força por unidade de área. Considerando o vetor $\overrightarrow{\mathrm{A}}$ de grandeza igual à área, e na direção normal à área, podemos afirmar:

$$
\overrightarrow{\mathrm{F}}=\overrightarrow{\mathrm{A}} \cdot \overrightarrow{\overrightarrow{\mathrm{T}}}=\mathrm{A}_{\mathrm{i}} \overrightarrow{\mathrm{e}}_{\mathrm{i}} \cdot \mathrm{T}_{\mathrm{jk}} \overrightarrow{\mathrm{e}}_{\mathrm{j}} \overrightarrow{\mathrm{e}}_{\mathrm{k}}=\delta_{\mathrm{ij}} \mathrm{A}_{\mathrm{i}} \mathrm{T}_{\mathrm{jk}} \overrightarrow{\mathrm{e}}_{\mathrm{k}}=\mathrm{A}_{\mathrm{i}} \mathrm{T}_{\mathrm{ik}} \overrightarrow{\mathrm{e}}_{\mathrm{k}}=\mathrm{F}_{\mathrm{k}} \overrightarrow{\mathrm{e}}_{\mathrm{k}} \text {, em que } \overrightarrow{\overrightarrow{\mathrm{T}}} \text { é a tensão. }
$$


$\therefore \mathrm{T}_{\mathrm{ik}}=\frac{\mathrm{F}_{\mathrm{k}}}{\mathrm{A}_{\mathrm{i}}}$, vemos que obedece à regra do quociente. ${ }^{12}$

No segundo volume, quando tratarmos da Relatividade Geral, veremos que o tensor curvatura, de quarta ordem, também satisfaz essas circunstâncias, obedecendo à regra do quociente. Também é importante salientar que um tensor de segunda ordem está associado com duas direções, e não uma, como o vetor, ou nenhuma, como o escalar. Assim, quando dois índices forem iguais, a tensão será compressão e quando forem diferentes, cisalhamento, tratando-se da diádica de tensões (ARIS, ARFKEN, WEBER).

\subsection{FLUXO DE UM VETOR}

Generalizamos o conceito de fluxo de um líquido para o fluxo de um vetor considerando uma grandeza vetorial $\vec{v}$ passando por uma secção $S$ e calculando seu fluxo.

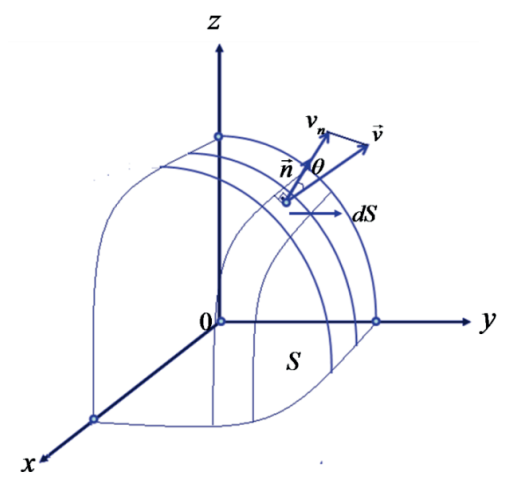

Fluxo elementar $d \phi$ em $d S$ : sendo $v_{n}$ componente de $\vec{v}$ na direção do vetor unitário $\vec{n}$, normal à secção $d S$, teremos: $\mathrm{d} \phi=\mathrm{v}_{\mathrm{n}} \mathrm{d} S=\mathrm{v} \cos \theta \mathrm{d} S$.

$$
\mathrm{d} \phi=\overrightarrow{\mathrm{v}} \cdot \overrightarrow{\mathrm{n}} \mathrm{d} S
$$

$\overrightarrow{\mathrm{n}}$ é o vetor unitário normal à superfície dS

Integrando em toda secção $S$ :

$$
\phi=\iint_{S} \vec{v} \cdot \vec{n} d S
$$

\subsection{CAMPO GRAVITACIONAL E CAMPO ELÉTRICO}

${ }^{12}$ Em uma equação tensorial, os índices do lado esquerdo devem aparecer no lado direito, exceto quando repetidos, quando desaparecem, indicando formação de escalar e também quando existir produto escalar, em que há contração de índices. Estamos nos referindo aos índices das componentes e não dos vetores unitários. 


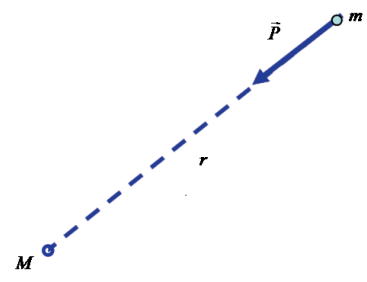

Seja um corpo de massa $M$ (como a Terra) que suporemos concentrada em seu centro. A uma distância $r$ colocamos várias massas: $m, m_{1}, m_{2}, m_{3} \ldots$ Essas massas ficarão sujeitas, respectivamente, às forças $\vec{P}, \vec{P}_{1}, \vec{P}_{2}, \vec{P}_{3} \ldots$ que chamaremos de peso. As relações entre peso e massa são constantes e iguais a $\vec{g}$ , isto é, à aceleração da gravidade: $\frac{\vec{P}}{m}=\frac{\vec{P}_{1}}{m_{1}}=\frac{\vec{P}_{2}}{m_{2}}=\frac{\vec{P}_{3}}{m_{3}}=\vec{g} \quad \therefore \bar{P}=m \vec{g}$.

Comparemos com a segunda lei de Newton: $\vec{F}=m \vec{a}$, nesse caso: $\vec{F}=\vec{P}$ e $\overrightarrow{\mathrm{a}}=\overrightarrow{\mathrm{g}}$

Pela lei da gravitação: $F=G \frac{m_{1} m_{2}}{r^{2}}$ e pela lei de Coulomb: $F= \pm \frac{1}{4 \pi \varepsilon_{0}} \frac{Q_{1} Q_{2}}{r^{2}}$, vemos semelhanças entre elas. São leis do inverso do quadrado da distância.

Logo, dada uma carga $Q$, ela produzirá um campo elétrico tal, que a uma distância $r$, se colocarmos várias cargas: $q, q_{1}, q_{2}, q_{3} \ldots$ essas ficarão sujeitas, respectivamente, às forças $\vec{F}, \vec{F}_{1}, \vec{F}_{2}, \vec{F}_{3} \ldots$

As relações entre força e carga são constantes e iguais a $\vec{E}$, "vetor campo elétrico": $\frac{\vec{F}}{q}=\frac{\vec{F}_{1}}{q_{1}}=\frac{\vec{F}_{2}}{q_{2}}=\frac{\vec{F}_{3}}{q_{3}}=\vec{E}$ em $N / C \quad \therefore \vec{F}=q \vec{E}$

\subsection{LINHAS DE FORÇA}

Uma linha de força é aquela em que cada ponto é tangente ao vetor campo nesse ponto.

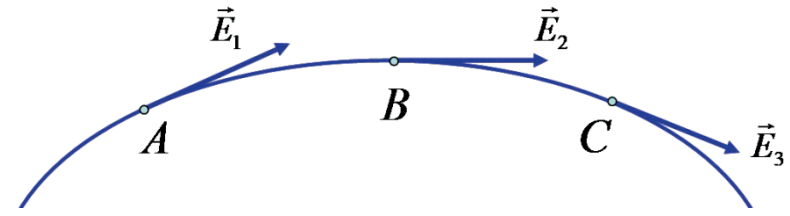

Portanto, indica a direção da força que surge em cada ponto quando se coloca uma massa ou uma carga nesse ponto (para campo gravitacional ou campo elétrico). Michael Faraday (1791-1867) iniciou sua utilização. 


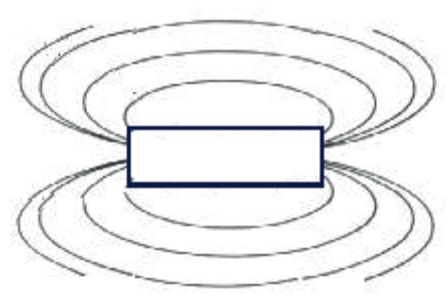

Podemos obter a visualização das linhas de força se colocarmos um cartão sobre uma barra imantada e, por cima do cartão, espalharmos limalha de ferro. Obteremos o que se chama de "espectro magnético".

As partículas de limalha se transformam em pequenos ímãs, os quais se orientam conforme as linhas de força do campo magnético. As linhas de força nunca se cruzam.

\subsection{VARIAÇÃO DO FLUXO DO VETOR: DIVERGÊNCIA}

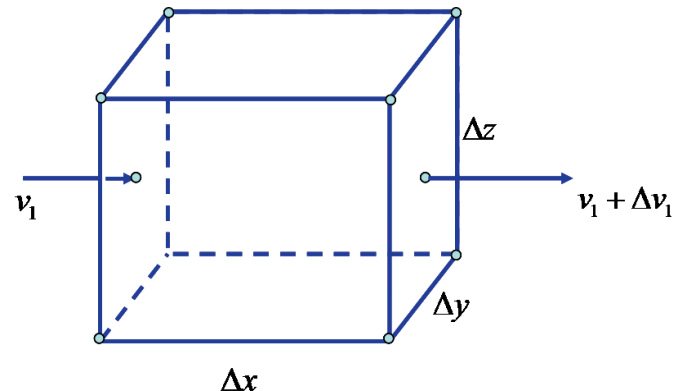

Seja um paralelepípedo elementar de lados $\Delta x, \Delta y, \Delta z$. Na face $\Delta y, \Delta z$ entra um fluxo $\phi_{1}$ com velocidade $v_{1}$, e na face oposta, sai: $\phi_{1}+\Delta \phi_{1}$ com velocidade $v_{1}+\Delta v_{1}$.

Variação do fluxo na direção $x$ :

$$
\Delta \phi_{1}=\Delta v_{1} \Delta y \Delta z, \Delta \phi_{1}=\frac{\Delta v_{1}}{\Delta x} \Delta x \Delta y \Delta z=\frac{\Delta v_{1}}{\Delta x} \Delta V
$$

$\Delta V$ Volume elementar $=\Delta \mathrm{x} \Delta \mathrm{y} \Delta \mathrm{z}$

Variação de fluxo total $\Delta \phi$ nas três direções $x, y, z$ :

$$
\Delta \phi=\Delta \phi_{1}+\Delta \phi_{2}+\Delta \phi_{3}=\left(\frac{\Delta v_{1}}{\Delta x}+\frac{\Delta v_{2}}{\Delta y}+\frac{\Delta v_{3}}{\Delta z}\right) \Delta V
$$


Variação do fluxo por unidade de volume:

$$
\frac{\Delta \phi}{\Delta V}=\frac{\Delta v_{1}}{\Delta x}+\frac{\Delta v_{2}}{\Delta y}+\frac{\Delta v_{3}}{\Delta z} \quad \text { Como: } \quad \Delta \phi=\iint_{S} \vec{v} \cdot \vec{n} d S
$$

(ver Seção 5.4).

Levando ao limite:

$\lim _{\Delta V \rightarrow 0} \frac{\oiint_{S} \vec{v} \cdot \vec{n} d S}{\Delta V}=\lim _{\Delta V \rightarrow 0}\left(\frac{\Delta v_{1}}{\Delta x}+\frac{\Delta v_{2}}{\Delta y}+\frac{\Delta v_{3}}{\Delta z}\right)=\frac{\partial v_{1}}{\partial x}+\frac{\partial v_{2}}{\partial y}+\frac{\partial v_{3}}{\partial z}=\frac{d \phi}{d V}$

Chamamos essa última expressão de divergência de $\vec{v}$ :

$\operatorname{div} \vec{v}=\frac{\partial v_{1}}{\partial x}+\frac{\partial v_{2}}{\partial y}+\frac{\partial v_{3}}{\partial z} \quad$ Em que: $\vec{v}=v_{1} \vec{i}+v_{2} \vec{j}+v_{3} \vec{k} \quad$ ou

$\operatorname{div} \vec{v}=\vec{\nabla} \cdot \vec{v}=\left(\frac{\partial}{\partial x} \vec{i}+\frac{\partial}{\partial y} \vec{j}+\frac{\partial}{\partial z} \vec{k}\right) \cdot\left(v_{1} \vec{i}+v_{2} \vec{j}+v_{3} \vec{k}\right)=\frac{\partial v_{1}}{\partial x}+\frac{\partial v_{2}}{\partial y}+\frac{\partial v_{3}}{\partial z}=\frac{d \phi}{d V}$

Em que: $\vec{\nabla}=\frac{\partial}{\partial x} \vec{x}+\frac{\partial}{\partial y} \vec{\jmath}+\frac{\partial}{\partial z} \vec{k}$

\subsection{TEOREMA DE GAUSS-OSTROGRADSKY}

Transforma integral tripla em um volume $V$ em uma integral dupla da superfície $S$ que encerra $V$.

${ }_{13}$ Recorde o produto escalar: $\vec{A} \cdot \vec{B}=\sum_{i, j=1}^{3} A_{i} B_{j} \vec{i} \cdot \vec{j}$, se $\mathrm{i}=\mathrm{j}, \overrightarrow{\mathrm{i}} \cdot \overrightarrow{\mathrm{j}}=\cos \alpha_{i j}=1$, pois o ângulo $\propto_{\mathrm{ij}}$ entre $\overrightarrow{\mathrm{i}}$ e $\vec{\jmath}$ é nulo; se $\overrightarrow{\mathrm{i}} \neq \overrightarrow{\mathrm{j}}, \overrightarrow{\mathrm{i}} \cdot \overrightarrow{\mathrm{j}}=0$, pois $\alpha_{i j}=90^{\circ}$.

Logo: $\vec{A} \cdot \vec{B}=A_{1} B_{1}+A_{2} B_{2}+A_{3} B_{3}$, pois $\vec{A}=A_{1} \overrightarrow{1}+A_{2} \vec{\jmath}+A_{3} \vec{k}$ e $\vec{B}=B_{1} \overrightarrow{1}+B_{2} \vec{\jmath}+B_{3} \vec{k}$ como os termos da soma são escalares, $o$ resultado da soma será um escalar.

$\sum_{i, j=1}^{3} A_{i} B_{j} \vec{i} \cdot \vec{j}$ 


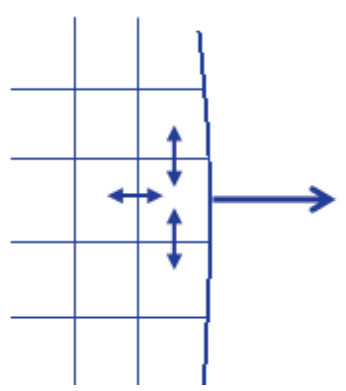

O volume $V$ é dividido em uma grande quantidade de paralelepípedos diferenciais. O fluxo em cada paralelepípedo será:

$$
\sum_{6 \text { superficies }} \overrightarrow{\mathrm{v}} \cdot \overrightarrow{\mathrm{n}} \mathrm{d} S=\vec{\nabla} \cdot \overrightarrow{\mathrm{v}} \mathrm{dV}
$$

Assim como visto na secção anterior, pois $\mathrm{d} \phi=\vec{\nabla} \cdot \overrightarrow{\mathrm{v}} \mathrm{dV}$ é a variação elementar do fluxo.

O fluxo interno é compensado pelas faces contíguas dos paralelepípedos. Os termos $\vec{v} \cdot \vec{n} d S$ se cancelam aos pares. Contudo, o fluxo externo não é compensado. ${ }^{14}$

Logo, o fluxo total será composto pelas faces externas que não são compensadas por não serem contíguas a outros paralelepípedos.

$$
\begin{array}{rr}
\sum_{\text {sup erf.externa }} \vec{v} \cdot \vec{n} d S=\sum_{\text {volunes }} \vec{\nabla} \cdot \vec{v} d V \text { Quando } n \rightarrow \infty \text { ou } d S \text { e } d V \rightarrow 0 \text {, teremos: } \\
\oiint \vec{v} \cdot \vec{n} d S=\iiint \vec{\nabla} \cdot \vec{v} d V & \begin{array}{r}
\text {, o círculo significa superfície fechada, } \\
\text { conforme Carl Friedrich Gauss (1777-1855). }
\end{array}
\end{array}
$$

Do parágrafo anterior: $\frac{\mathrm{d} \phi}{\mathrm{dV}}=\vec{\nabla} \cdot \overrightarrow{\mathrm{v}} \Rightarrow \mathrm{d} \phi=\vec{\nabla} \cdot \overrightarrow{\mathrm{v}} \mathrm{dV}$, integrando:

\footnotetext{
${ }^{14}$ Supondo não haver fontes nem sumidouros. São consideradas singularidades. Nesse caso, teremos acréscimos ou decréscimos de fluxo interno que deverão ser considerados. No segundo volume, esclareceremos o significado de singularidade (ver curvatura do espaço segundo Einstein em Sagan e singularidade, conforme Hawking em Uma breve história do tempo). Para melhor compreensão das singularidades, ver a Seção 5.25, sobre a "distribuição da matéria".
} 


$$
\Delta \phi=\oiint_{S} \overrightarrow{\mathrm{v}} \cdot \overrightarrow{\mathrm{n}} \mathrm{d} S=\oiiint_{\mathrm{V}} \vec{\nabla} \cdot \overrightarrow{\mathrm{v}} \mathrm{dV},
$$

concordando com a dedução mencionada anteriormente. Ou seja, o fluxo $\Delta \phi$ da função vetorial $\vec{v}$, através da superfície de controle SC, resulta na divergência dessa função no volume de controle VC, encerrado pela superfície de controle SC.

\section{Observação}

Há uma demonstração tensorial no assunto quando falarmos sobre o vetor de Poynting na Seção 5.26, Equação (5.10).

\subsection{EQUAÇÃO DA CONTINUIDADE}

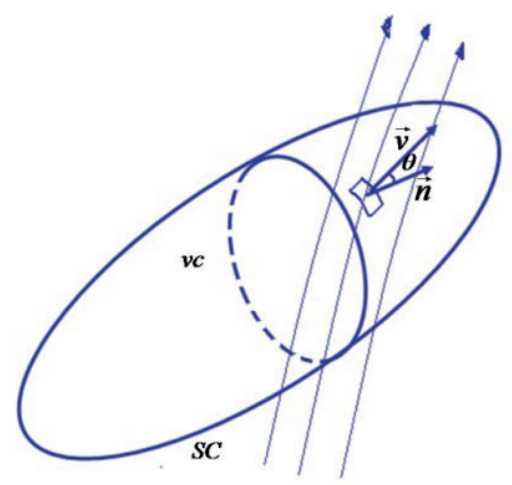

Seja o volume de controle $V C$, envolvido pela superfície de controle $S C$. A variação em relação ao tempo da massa ou carga no $V C$ será dada por: $\frac{\partial}{\partial t} \iiint_{V} \rho d V$, em que $\rho=\frac{d m}{d V}$ ou $\frac{d q}{d V}(0)$

Ou seja, massa ou carga por unidade de volume.

A variação é devida aos fluxos de entrada e saída da massa ou carga através da superfície de controle $S C$, que será calculada por: $\oiint_{S} \rho \vec{v} \cdot \vec{n} d S .^{15}$

\footnotetext{
${ }^{5}$ Análise dimensional de fluxo: verifique Seção $5.4, \vec{v} \cdot \overrightarrow{\mathrm{n}} \mathrm{dS} \Rightarrow([\mathrm{L}] /[\mathrm{T}])\left[\mathrm{L}^{2}\right]=\left[\mathrm{L}^{3}\right] /[\mathrm{T}] \cdot{ }^{*}$, que é vazão, isto é, volume na unidade de tempo. $\rho \overrightarrow{\mathrm{v}} \cdot \overrightarrow{\mathrm{n}} \mathrm{dS} \Rightarrow\left([\mathrm{M}] /\left[\mathrm{L}^{3}\right]\right)\left(\left[\mathrm{L}^{3}\right] /[\mathrm{T}]\right)=[\mathrm{M}] /[\mathrm{T}]$, massa na unidade de tempo ou: $\left([\mathrm{Q}] /\left[\mathrm{L}^{3}\right]\right)\left(\left[\mathrm{L}^{3}\right] /[\mathrm{T}]\right)=[\mathrm{Q}] /[\mathrm{T}]$ carga na unidade de tempo e $\partial / \partial \mathrm{t}(\rho \mathrm{dV}) \Rightarrow \rho \mathrm{dV}=\mathrm{dm}$ ou dq $\therefore$ massa ou carga na unidade de tempo, ou seja, corrente i, como será visto na Seção 5.16 .
} 
O círculo significa que é calculado na superfície fechada.

Nota-se que o fluxo de saída é positivo, pois $\theta<90^{\circ}$ e $\cos \theta>0$. O fluxo de entrada é negativo, pois $\theta>90^{\circ}$ e $\cos \theta<0$. Recorde-se que $\overrightarrow{\mathrm{v}} \cdot \overrightarrow{\mathrm{n}}=\cos \theta$.

Então teremos: $\frac{\partial}{\partial t} \iiint_{V} \rho d V=-\oiint_{S} \rho \vec{v} \cdot \vec{n} d S$

O sinal negativo indica que, quando o fluxo entra e, portanto, é negativo, a quantidade de carga ou massa aumenta e, logo, o acréscimo é positivo. Quando o fluxo sai, teremos o oposto, isto é, o fluxo é positivo, mas haverá um decréscimo na massa ou carga. Trata-se de um balanço material ou de cargas no $V C$.

Essa é a equação integral da continuidade.

Levando em conta o teorema de Gauss-Ostrogradsky, ou seja:

$$
\oiint_{S} \vec{v} \cdot \vec{n} d S=\iiint_{V} \vec{\nabla} \cdot \vec{v} d V
$$

Teremos, substituindo $\vec{v}$ por $\rho \vec{v}$, nessa equação.

$$
\iiint_{V} \vec{\nabla} \cdot(\rho \vec{v}) d V=-\frac{\partial}{\partial t} \iiint_{V} \rho d V, \text { pois: } \quad \oiint \int_{S} \rho \vec{v} \cdot \vec{n} \mathrm{~d} S=\iiint_{V} \vec{\nabla} \cdot(\rho \vec{v}) \mathrm{dV}
$$

\subsubsection{Demonstração da equivalência}

$$
\begin{aligned}
& \int_{S} \rho \vec{v} \cdot \vec{n} d S=\int_{S} \rho v_{n} d S \\
& \mathrm{v}_{\mathrm{n}}=\overrightarrow{\mathrm{v}} \cdot \overrightarrow{\mathrm{n}} \text {, velocidade perpendicular a dS, aplicando Gauss- } \\
& \text { Ostrogradski: } \\
& \int_{S} \rho \vec{v} \cdot \vec{n} d S=\int_{V} \vec{\nabla} \cdot(\rho \vec{v}) d V=\int_{V}\left(\frac{\partial}{\partial x_{i}} \vec{e}_{i}\right) \cdot\left(\rho \frac{\partial x_{j}}{\partial t} \vec{e}_{j}\right) d V=\int_{V} \rho\left(\frac{\partial}{\partial x_{i}}\right) \delta_{i j}\left(\frac{\partial x_{j}}{\partial t}\right) d V= \\
& \therefore \int_{S} \rho \overrightarrow{\mathrm{v}} \cdot \overrightarrow{\mathrm{n}} \mathrm{d} S=\frac{\partial}{\partial \mathrm{t}} \int_{\mathrm{V}} \rho \mathrm{dV} \\
& =\int_{V} \rho\left(\frac{\partial}{\partial x_{i}}\right)\left(\frac{\partial x_{i}}{\partial t}\right) d V=\frac{\partial}{\partial t} \int_{V} \rho d V
\end{aligned}
$$


A menos do sinal que já demonstramos no $2^{\circ}$ membro, ser negativo.

$$
\frac{\partial}{\partial t} \oint_{V} \rho d V=-\oint_{S} \rho \vec{v} \cdot \vec{n} d S
$$

Em um VC fechado, quando há aumento do fluxo (positivo) no VC, a massa ou carga na SC diminui (negativo) e o oposto quando houver diminuição do fluxo (ver a figura da Seção 5.9).

A fórmula é válida independentemente do volume de integração. Logo, tendo em mente que a derivação é a operação inversa da integração: ${ }^{16}$

$$
\vec{\nabla} \cdot(\rho \vec{v})=-\frac{\partial \rho}{\partial t} \quad \therefore \frac{\partial\left(\rho v_{1}\right)}{\partial x}+\frac{\partial\left(\rho v_{2}\right)}{\partial y}+\frac{\partial\left(\rho v_{3}\right)}{\partial z}=-\frac{\partial \rho}{\partial t} \text { (ver observação ao pé da página na Seção 5.7) }
$$

É a equação da continuidade em forma diferencial.

\subsection{LEI DE GAUSS PARA ELETRICIDADE}

O fluxo vetorial não é necessariamente material.

$$
\phi=\iint_{S} \vec{v} \cdot \vec{n} d S
$$

No caso de fluxo elétrico teremos:

${ }^{16}$ Pois: a área A sob a curva $\mathrm{y}=\mathrm{f}(\mathrm{x})$, no intervalo $\mathrm{a} \leqq \mathrm{x} \leqq \mathrm{b}$, é dada pela integral $\mathrm{A}=\int_{\mathrm{a}}^{\mathrm{b}} \mathrm{ydx}$, derivando: $\frac{d}{d x} \int_{a}^{b} y d x=\frac{d}{d x} A=\frac{d A}{d x}$, significa, $a \rightarrow b$ (“a” tende a " $b$ ") e a integral se torna $d A=y d x$, obtemos:

$$
\frac{d \int_{a}^{b} y d x}{d x}=\lim _{\mathrm{n} \rightarrow \infty} \frac{\Delta \int_{a}^{b} y d x}{\Delta x}=\frac{y d x}{d x}=y
$$

$\Delta$ é variação. No lim, o que era uma variação $\Delta$, se torna um infinitésimo d. Observe que a operação diferencial $d$ anula a operação integral $\int$.

Tomando-se a área elementar dA, obtida multiplicando a ordenada y pelo elemento da abcissa dx, ou seja, dA = ydx, e integrando entre os limites $\mathrm{a} \leqq \mathrm{x} \leqq \mathrm{b}$, recuperamos a área inicial.

$$
A=\int_{a}^{b} y d x=\lim _{\Delta x \rightarrow 0} \sum_{x_{i}=\mathrm{a}}^{b} y_{i} \Delta x_{i} \quad \text { Sendo } \quad d x=\lim _{\Delta x \rightarrow 0} \Delta x
$$

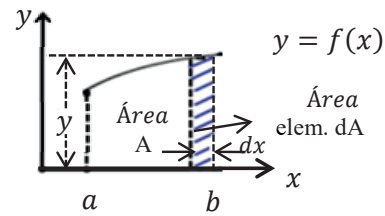

Simplificando: seja a área $A$, calculada pela integral $\mathrm{A}=\int_{\mathrm{a}}^{\mathrm{b}} \mathrm{ydx}$. Achar a função $\mathrm{y}=\mathrm{f}(\mathrm{x})$. Para isso, calculamos a derivada da integral:

$$
y=\frac{d}{d x} \int_{a}^{b} f(x) d x=f(x)
$$




$$
\phi_{e}=\iint_{S} \overrightarrow{\mathrm{E}} \cdot \vec{n} d S=\iiint_{V} \vec{\nabla} \cdot \overrightarrow{\mathrm{E}} d V
$$

A partir disso, obtemos: $\mathrm{E}=\frac{\mathrm{d} \phi_{\mathrm{e}}}{\mathrm{dS}}$, em que E, campo elétrico, da Seção 5.6, conceito de "Linhas de força", pode-se afirmar que a quantidade de linhas de força é deduzida pela relação do fluxo $\phi_{\mathrm{e}} \mathrm{em} \mathrm{N} / \mathrm{C}$ pela superfície $\mathrm{S}$ em $\mathrm{m}^{2}$, no sistema MKS. Ver também Seção 5.17a, potencial elétrico.

Utilizamos anteriormente o teorema de Gauss-Ostrogradsky (ver final da Seção 5.8), porém, $\overrightarrow{\mathrm{E}}=\frac{\vec{F}}{q_{0}}=\frac{q}{4 \pi \varepsilon_{0} r^{2}} \vec{u}_{r}{ }^{17} \quad q_{0}$ - carga de prova sujeita à força $\vec{F}$ a uma distância $r$ de $q, \vec{u}_{r}$ - vetor unitário.

Cada linha de força se origina em uma carga positiva e termina em uma carga negativa de mesmo valor absoluto.

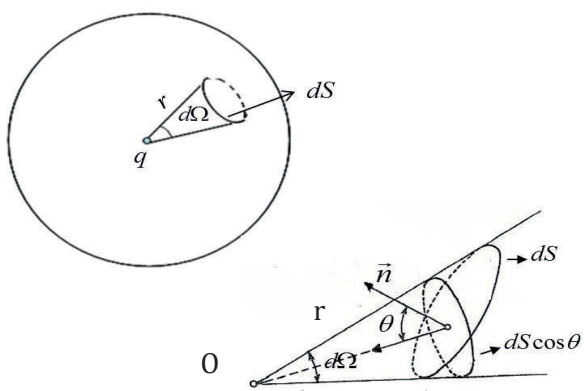

Carga $q$ no interior de uma superfície fechada $S$.

$d \Omega$ : ângulo sólido em esferorradianos.

$$
\begin{array}{r}
\phi_{\mathrm{e}}=\oint_{S} \mathrm{E} \cos \theta \mathrm{d} S=\oint_{S} \frac{\mathrm{q}}{4 \pi \varepsilon_{0} \mathrm{r}^{2}} \mathrm{~d} S \cos \theta \\
\phi_{\mathrm{e}}=\frac{\mathrm{q}}{4 \pi \varepsilon_{0}} \oint_{S} \mathrm{~d} \Omega=\frac{\mathrm{q}}{4 \pi \varepsilon_{0}}(4 \pi)=\frac{\mathrm{q}}{\varepsilon_{0}}
\end{array}
$$

Contudo, dS $\cos \theta / \mathrm{r}^{2}$ é o ângulo sólido $d \Omega$.

\footnotetext{
${ }^{17}$ Ver Lei de Coulomb, Seção 5.1: $\mathrm{F}=\frac{1}{4 \pi \varepsilon_{0}} \frac{\mathrm{qq} \mathrm{q}_{\mathrm{o}}}{\mathrm{r}^{2}}$.
} 
Pois o ângulo sólido total é $4 \pi$. Considerando que a superfície da esfera é $4 \pi r^{2}$, logo:

$$
\Omega=4 \pi r^{2} / r^{2}(1)^{18}
$$

A fórmula é válida também para $n$ cargas.

Então teremos: $\phi_{e}=\iiint_{V} \vec{\nabla} \cdot \vec{E} d V=\frac{q}{\varepsilon_{0}}(0) \quad$ Lei de Gauss $\quad \int_{\mathrm{S}} \overrightarrow{\mathrm{E}} \cdot \overrightarrow{\mathrm{n}} \mathrm{d} S=\frac{\mathrm{q}}{\varepsilon_{0}} \quad$ e equação $1 \mathrm{a}$ por Gauss- Ostrogradsky.

Se fizermos $q=\iiint_{V} \rho d V$, teremos: $\vec{\nabla} \cdot \vec{E}=\frac{\rho}{\varepsilon_{0}} \quad$ em forma diferencial, explicitando:

$\frac{\partial E_{1}}{\partial x}+\frac{\partial E_{2}}{\partial y}+\frac{\partial E_{3}}{\partial z}=\frac{\rho}{\varepsilon_{0}}$ Também chamada de $1^{\text {a }}$ lei de Maxwell (ALONSO, FINN, SALMERON).

O fluxo elétrico $\phi_{e}$ através de uma superfície fechada é proporcional à soma das cargas elétricas no interior dessa superfície. Se não houver carga: $\vec{\nabla} \cdot \vec{E}=0$ (2). Indica que as fontes do fluxo elétrico são as cargas elétricas (YAVORSKI).

18 Definimos o radiano em um ângulo plano como a relação entre o arco $\ell$ e o raio R (ver Figura a seguir). Um "ângulo sólido" é o espaço compreendido no interior de uma superfície cônica ou piramidal. Em analogia ao ângulo em radianos, definimos o esferorradiano, sr, como a relação entre a superfície $S$ intersecção do ângulo sólido da esfera com o centro no vértice da superfície e seu raio $R$ ( ver Figura anterior). Como a esfera tem uma superfície de $4 \pi R^{2}$, essa relação se torna: $\Omega=S / R^{2}=$ $4 \pi$ = ângulo sólido da esfera. Se o ângulo sólido for pequeno, ou seja, elementar $\mathrm{d} \Omega$, pode-se substituir a calota esférica $\mathrm{S}$ por uma superfície plana elementar $\mathrm{dS}: \mathrm{d} \Omega=\mathrm{dS} / \mathrm{R}^{2}$.

Se dS não for perpendicular ao eixo do ângulo sólido, projetamos essa superfície sobre uma superfície perpendicular ao eixo, $\mathrm{dS}^{\prime}=\mathrm{dS} \cos \theta$ (ver Figura anterior), obtendo-se: $\mathrm{d} \Omega=(\mathrm{dS} \cos \theta) / \mathrm{R}^{2}$, como visto anteriormente (ALONSO, FINN). Sobre a superfície da esfera, ver Anexo 10.

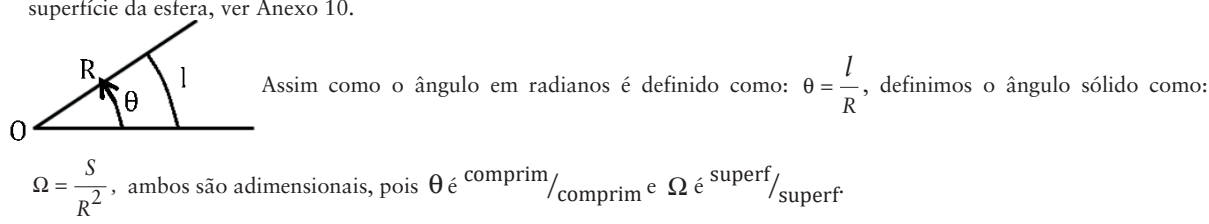




\subsection{INDUÇÃO MAGNÉTICA}

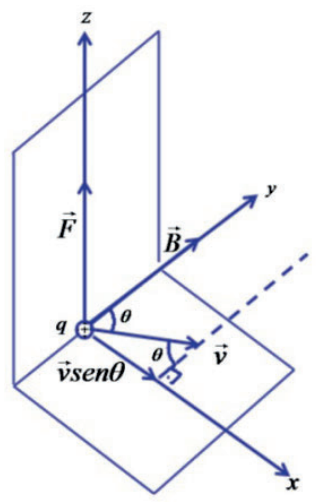

Se uma carga $q$ com velocidade $\vec{v}$ passar por um campo magnético de vetor de indução $\vec{B}$, ficará sujeita a uma força perpendicular a $\vec{v}$ e a $\vec{B}$, obedecendo à equação vetorial:

$$
\vec{F}=q \vec{v} \times \vec{B}
$$

Logo, a indução magnética será: $\quad B=\frac{F}{q v \operatorname{sen} \theta}$

Se a carga estiver sujeita ao campo magnético e ao campo elétrico, então ficará sujeita à força total (soma das forças elétrica e magnética), ou seja,

$$
\vec{F}=q \vec{E}+q \vec{v} \times \vec{B}
$$

Denominada Força de Lorentz.

\subsection{LEI DE GAUSS PARA O MAGNETISMO}

O fluxo magnético será definido como:

$$
\phi_{B}=\iint_{S} \vec{B} \cdot \vec{n} d S=\iint_{S} B \cos \theta
$$

Para uma superfície fechada, isto é, $V C \operatorname{com} S C$ fechada: $\phi_{B}=\oiint_{S} \vec{B} \cdot \vec{n} d S=0$

É impossível obter um único polo magnético, pois eles sempre trabalham aos pares. 
Forma diferencial:

$$
\oiint_{S} \vec{B} \cdot \vec{n} d S=\iiint_{V} \vec{\nabla} \cdot \vec{B} d V=0
$$

Por Gauss-Ostrogradsky, ver Seção 5.8.

Logo: $\vec{\nabla} \cdot \vec{B}=0$ ou $\frac{\partial B_{1}}{\partial x}+\frac{\partial B_{2}}{\partial y}+\frac{\partial B_{3}}{\partial z}=0$, a $2^{\text {a }}$ lei de Maxwell.

As linhas de força do campo magnético emergem externamente do polo norte do imã e penetram no polo sul do imã. Internamente, as linhas de forças se dirigem do polo sul ao polo norte, tornando a linha de força magnética fechada. Por esse motivo, o divergente da indução magnética $\vec{B}$ é nulo: $\vec{\nabla} \cdot \vec{B}=0$. Comparando com a lei de Gauss para eletricidade (Seção 5.10): $\varepsilon_{0} \vec{\nabla} \cdot \overrightarrow{\mathrm{E}}=\mathrm{q}$, verificamos que carga magnética não existe.

Tomando-se o fluxo magnético:

$$
\phi_{\mathrm{B}}=\oint_{\mathrm{S}} \overrightarrow{\mathrm{B}} \cdot \overrightarrow{\mathrm{n}} \mathrm{d} S
$$

Vemos que o fluxo para fora é positivo e o fluxo para dentro tem o mesmo valor, porém negativo, sendo a soma dos dois, nula. ${ }^{19}$ Essa a verificação é experimental (HALLIDAY, RESNICK).

\subsection{DIFERENÇA DE POTENCIAL}

A energia potencial entre dois pontos $a$ e $b$ será:

$$
E_{p}=\int_{a}^{b} F \cos \alpha d l=\int_{a}^{b} q(E+B v) \cos \alpha d l
$$

(Se $\vec{B}$ e $\vec{v}$ forem perpendiculares)

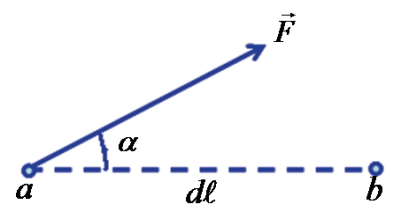

9 Indica que na natureza não há cargas magnéticas e que as linhas de indução do campo magnético são fechadas. O que temos são dipolos magnéticos, como por exemplo, no campo magnético produzido pelo spin dos elétrons, que funcionam como imãs elementares (YAVORSKI). Como veremos adiante na subseção 5.14.1 equação 1. 
Pois, pela força de Lorentz: $\frac{F}{q}=E+B v$ [ver Seção 5.11, Equação (2)].

Chamamos diferença de potencial a: $\frac{E_{p}}{q}=\Delta V_{a b}$

$\Delta V_{a b}=\int_{a}^{b}(E+B v) \cos \alpha d \ell \quad$ (para $\vec{B}$ e $\vec{v}$ perpendiculares)

\subsection{LEI DE FARADAY-HENRY}

Foi deduzida por Michael Faraday em 1831.

Indução magnética $\perp$ ao plano: $\vec{B}$, apontando para para fora na direção do leitor.

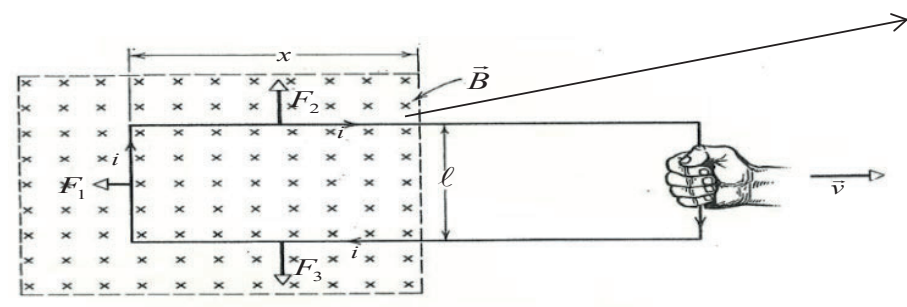

O movimento do circuito fechado com velocidade $\vec{v}$ induz uma corrente $i$ no circuito. Na Seção 5.11, o sistema de referência estava em $\overrightarrow{\mathrm{B}}$. Agora, o sistema de referência está na carga q, movendo-se no circuito. Logo, é $\overrightarrow{\mathrm{B}}$ que se move em relação a q.

O potencial será: $\boldsymbol{\Delta} \boldsymbol{V}=\boldsymbol{\oint}_{\boldsymbol{c}}(\boldsymbol{E}+\boldsymbol{B} \boldsymbol{v}) \cos \boldsymbol{\alpha} \boldsymbol{d} \quad$ (0) (ver Seção 5.13).

Como o circuito é fechado, o potencial elétrico será nulo, pois $a=b$ e $\Delta V_{a b}=0$ (potencial elétrico).

Em nosso caso, $\vec{F}_{2}=-\vec{F}_{3}$ e se cancelam. 
$\vec{F}_{1}$ será função de $\theta=90^{\circ}, \log 0, \operatorname{sen} \theta=1^{20} \quad$ e $\quad \Delta V=B \ell v^{21}$

$\theta$ é o ângulo entre $\vec{B}$ e $\vec{v}$.

Como $\phi_{B}=B \ell x \quad \therefore \frac{d \phi_{B}}{d t}=-\frac{d}{d t}(B \ell x)=-B \ell \frac{d x}{d t}=-B \ell v$

(B $\times$ superfície $)$

$\therefore \Delta \mathrm{V}=-\frac{\mathrm{d} \phi_{\mathrm{B}}}{\mathrm{dt}}(1)$

(Lei de Faraday-Henry)

Sendo $\phi_{\mathrm{B}}$, fluxo magnético.

A uma variação positiva de dt (variação elementar de tempo) corresponde uma variação negativa da área $\ell \mathrm{x}$, então, deve-se usar o sinal negativo.

\subsubsection{Interpretação do sinal negativo na lei de Faraday-Henry}

Sistema dextrogiro

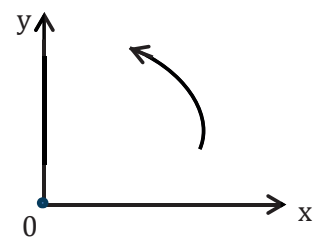

${ }^{20} F_{1}=-q u B$ ver Equação (1), Seção 5.11: $\mathrm{F}_{1}=-q u B \operatorname{sen} \theta \therefore-E=-\frac{F_{1}}{q}=u B, \mathrm{E}+\mathrm{uB}=0, \mathrm{u}=\frac{\mathrm{dl}}{\mathrm{dt}}$, é $\perp$ a: $\mathrm{v}=\frac{\mathrm{dx}}{\mathrm{dt}}$.

Pela regra da mão esquerda: $\vec{F} \perp$ a $\vec{B}$ e a $\vec{w}$

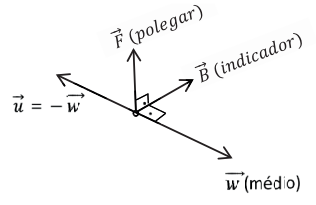

${ }^{21}$ Devido à corrente circulante $i$, surge força no condutor móvel. $F_{1}=B \ell i$ pois: $F_{1}=q u B \Rightarrow d F_{1}=d q \frac{d \ell}{d t} B=i B d \ell \mathrm{e}$ $F_{1}=\int d F_{1}=\int B i d \ell=B \ell i$; distância $\quad d x=v d t$ trabalho $\quad$ realizado: $\quad d W=F_{1} d x=B \ell v i d t, \quad i d t=d q$ $\therefore d W=B \ell v d q$ potencial $\Delta V=d W / d q=B \ell v(1 \mathrm{a})$. 
Sistema dextrogiro

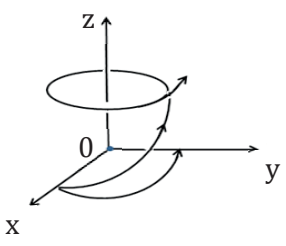

Produto vetorial

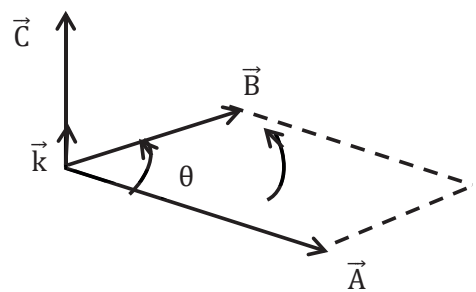

$\overrightarrow{\mathrm{C}}=\overrightarrow{\mathrm{A}} \times \overrightarrow{\mathrm{B}}=\mathrm{AB}(\operatorname{sen} \theta) \overrightarrow{\mathrm{k}}, \overrightarrow{\mathrm{k}}=$ vetor unitário

A rotação A, B, C é dextrogira (COURANT).

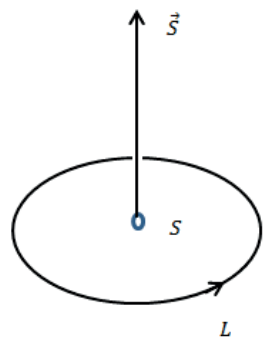

Se $\vec{A} \times \vec{B}=\vec{S} \cdot$, L contorno da área

Pela regra da mão direita, polegar para cima; outros dedos: direção contrarrelógio, isto é, sistema dextrogiro (Alonso Finn). 


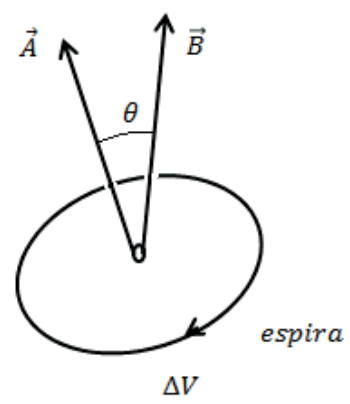

Com relação ao fluxo:

$\Delta V$ tem direção contrária à rotação dextrogira, então será negativo: $\phi_{\mathrm{B}}=\int \overrightarrow{\mathrm{B}} \cdot \mathrm{d} \overrightarrow{\mathrm{A}} \Rightarrow \frac{\mathrm{d} \phi_{\mathrm{B}}}{\mathrm{dt}}=-\Delta \mathrm{V}$

A direção de $\Delta \mathrm{V}$ foi determinada experimentalmente por Faraday, Henry e Lenz, de modo independente (HALLIDAY, RESNICK, WALKER). ${ }^{22}$

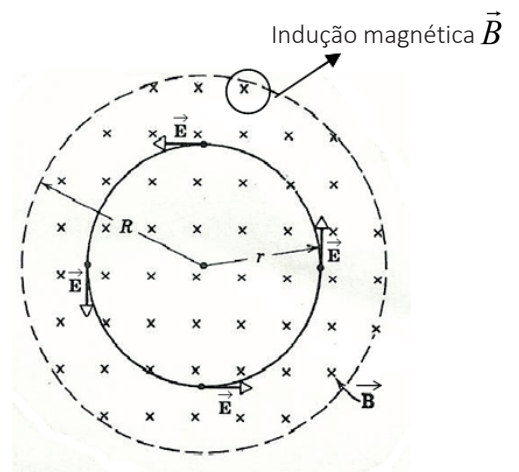

Seja uma carga q percorrendo o circuito com um campo induzido $\overrightarrow{\mathrm{E}}$ provocado por uma indução magnética variável $\overrightarrow{\mathrm{B}}$. Então o trabalho em cada rotação é:

$q \Delta V, \Delta \mathrm{V}=\mathrm{E}_{\mathrm{p}} / \mathrm{q}$, ver Seção 5.13.

Também deve ser igual a $(q E)(2 \pi r)$, pois $\mathrm{F}=\mathrm{qE}$.

22 Considerando a carga de q positiva. Quando $\phi_{\mathrm{B}}$ cresce, $\mathrm{d} \phi_{\mathrm{B}} / \mathrm{dt}$ é positivo e $\Delta \mathrm{V}$, negativo. Quando $\phi_{\mathrm{B}}$ decresce, teremos a variação do potencial $\Delta \mathrm{V}$ positiva. 
Energia potencial $\mathrm{E}_{\mathrm{p}}=$ trabalho: $\mathrm{F} \mathrm{x}$ distância (nesse caso, circunferência)

Logo: $\Delta V=E 2 \pi r$

No caso geral, devemos ter: $\Delta V=\oint \vec{E} \cdot d \vec{\ell}$

Então a lei da indução de Faraday, ver em (1), se torna:

$\oint \vec{E} \cdot \overrightarrow{d \ell}=-\frac{d \phi_{B}}{d t}$

A $3^{\text {a }}$ lei de Maxwell

O campo magnético variável com o tempo produz um campo elétrico, fenômeno chamado de “indução". Ele é utilizado, por exemplo, em um gerador elétrico, ou um dínamo, em que a variação do campo magnético é produzida por movimentação do magneto.

Retornando à questão do sinal, podemos resolvê-lo matematicamente: Tomando-se a primeira equação $(0): \quad \Delta V=\oint(E+B v) \cos \alpha \mathrm{d} \ell, \quad \operatorname{como} \quad \alpha=0^{\circ} \quad$ e $\quad$ o $\quad$ ciclo
fechado, temos:

$$
\Delta \mathrm{V}=\oint_{\mathrm{C}}(\mathrm{E}+\mathrm{Bv}) \mathrm{d} \ell=0 \Rightarrow \oint \overrightarrow{\mathrm{E}} \cdot \mathrm{d} \vec{\ell}=-\oint \operatorname{Bvd} \ell
$$

A $1^{\mathrm{a}}$ parcela chamaríamos de $\Delta \mathrm{V}_{\mathrm{E}}$, potencial elétrico, e a $2^{\mathrm{a}}, \Delta \mathrm{V}_{\mathrm{M}}$, de potencial magnético.

$\therefore \Delta V_{\mathrm{E}}=\oint \overrightarrow{\mathrm{E}} \cdot \mathrm{d} \vec{\ell}$

$\Delta \mathrm{V}_{\mathrm{M}}=\oint \mathrm{Bv} \mathrm{d} \ell$, como vimos ao pé da página na Seção 5.14, Equação (1a), integrando $\Delta \mathrm{V}=\mathrm{B} \ell \mathrm{v}$.

Logo: $\Delta \mathrm{V}_{\mathrm{E}}=-\Delta \mathrm{V}_{\mathrm{M}}$, justificando o sinal negativo.

Nada mais é do que obedecer ao princípio da "Conservação da energia” (HALLIDAY, RESNICK, WALKER).

\subsection{TEOREMA DE STOKES}

Transforma uma integral de linha de caminho fechado em uma integral da superfície circunscrita por essa linha.

Circulação: 


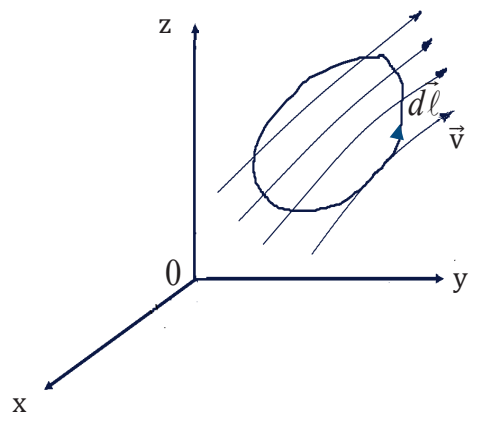

É uma integral de linha em um caminho fechado da componente tangencial ao longo da trajetória.

$$
\Gamma=\oint_{c} \vec{v} \cdot d \vec{\ell}
$$

Cálculo da circulação em um elemento de área $d x d y$ :

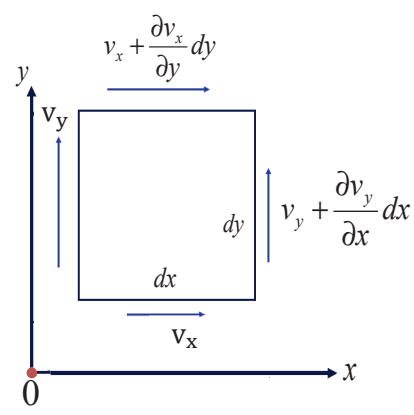

Circulação nos lados da área:

$1^{\circ}$ lado: $v_{x} d_{x}$

$2^{\circ}$ lado: $\left(v_{y}+\frac{\partial v_{y}}{\partial x} d x\right) d y$

3. lado: $-\left(v_{x}+\frac{\partial v_{x}}{\partial y} d y\right) d x$

$4^{\circ}$ lado: $-v_{y} d y$ 
Somando os resultados parciais, teremos: $\oint_{C} \vec{v} \cdot \overrightarrow{d \ell}=\left(\frac{\partial v_{y}}{\partial x}-\frac{\partial v_{x}}{\partial y}\right) d x d y$.

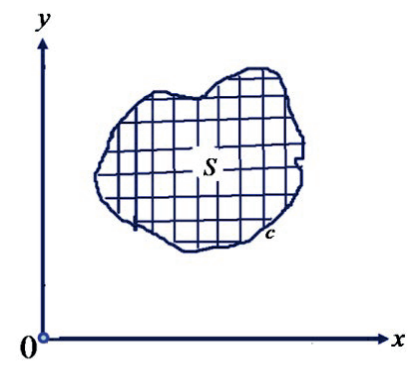

Porém, $\quad(\operatorname{rot} \overrightarrow{\mathrm{v}})_{\mathrm{z}}=(\vec{\nabla} \times \overrightarrow{\mathrm{v}})_{\mathrm{z}}=\left|\begin{array}{cc}\frac{\partial}{\partial \mathrm{x}} & \frac{\partial}{\partial \mathrm{y}} \\ \mathrm{v}_{\mathrm{x}} & \mathrm{v}_{\mathrm{y}}\end{array}\right|=\frac{\partial \mathrm{v}_{\mathrm{y}}}{\partial \mathrm{x}}-\frac{\partial \mathrm{v}_{\mathrm{x}}}{\partial \mathrm{x}}(1)$

Componente na direção $z$ do rotacional de $\vec{v}$ no plano $x y$

Logo: $\mathrm{d} \Gamma=\oint_{C} \vec{v} \cdot d \vec{\ell}=(\operatorname{rot} \vec{v})_{\mathrm{z}} d S$ Em que $\mathrm{d} S=\mathrm{dxdy}$

É uma circulação elementar.

Considerando uma área $S$, dividida em áreas elementares.

As circulações internas se cancelam mutuamente, restando a circulação no contorno $c$ da área $S$.

O raciocínio é semelhante ao utilizado para o teorema Gauss-Ostrogradsky da Seção 5.8.

$\operatorname{Logo:} \Gamma=\oint_{c} \vec{v} \cdot \overrightarrow{d \ell}=\iint_{S}(\operatorname{rot} \vec{v})_{\mathrm{z}} d S$

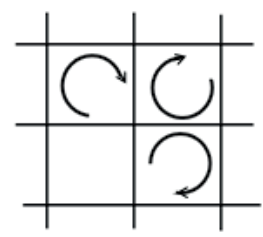

Trata-se do teorema bidimensional de Stokes, também chamado de Green.

Consideremos o caso tridimensional. Seja uma superfície $S$, limitada por uma curva não coplanar $c$. A superfície é subdividida em elementos infinitesimais de área $d:$. 


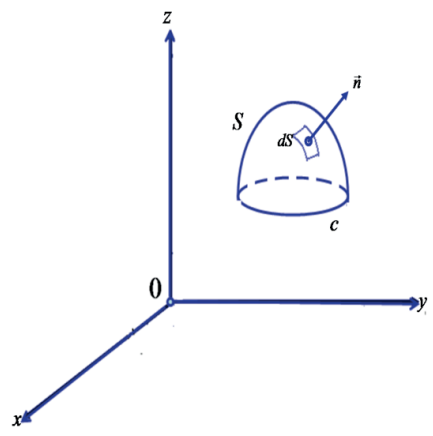

Para cada elemento de área, teremos:

$$
d \Gamma=(\operatorname{rot} \vec{v})_{n} d S=\operatorname{rot} \vec{v} \cdot \vec{n} d S
$$

Em que a componente do rotacional é normal ao elemento de área.

Da mesma forma como na divergência, as circulações internas se cancelam em $S$, restando as externas em $c$. Na divergência, os fluxos se cancelavam aos pares, agora, as circulações se cancelam (ver Seção 5.8 teorema de Gauss-Ostrogradsky).

Logo, teremos: $\oint_{c} \vec{v} \cdot d \vec{\ell}=\iint_{S}(\vec{\nabla} \times \vec{v}) \cdot \vec{n} d S$

Projetando nos planos $x y, y z$ e $z x$ :

$$
\oint_{C} v_{1} d x+v_{2} d y+v_{3} d z=\iint_{S}\left(\frac{\partial v_{2}}{\partial x}-\frac{\partial v_{1}}{\partial y}\right) d x d y+\left(\frac{\partial v_{3}}{\partial y}-\frac{\partial v_{2}}{\partial z}\right) d y d z+\left(\frac{\partial v_{1}}{\partial z}-\frac{\partial v_{3}}{\partial x}\right) d x d z
$$

Em que $d \vec{l}=d x \vec{\imath}+d y \vec{\jmath}+d z \vec{k}^{23}$

Aplicando na lei de Faraday (Seção 5.14) e utilizando o fluxo magnético (Seção 5.12), teremos:

$$
\iint_{S}(\vec{\nabla} \times \overrightarrow{\mathrm{E}}) \cdot \overrightarrow{\mathrm{n}} \mathrm{dS}=-\frac{\mathrm{d}}{\mathrm{dt}} \iint_{\mathrm{S}} \overrightarrow{\mathrm{B}} \cdot \overrightarrow{\mathrm{n}} \mathrm{dS} \quad \text { Logo: } \vec{\nabla} \times \vec{E}=-\frac{d \vec{B}}{d t}
$$

\footnotetext{
${ }^{23} \vec{v} \cdot d \vec{l}=v_{1} d x+v_{2} d y+v_{3} d z$, produto escalar, ver nota ao pé da página da Seção 5.7 .

${ }^{24}$ Trata-se de um vetor. Recordando o produto vetorial: se $i=j, \vec{i} \times \vec{j}=\operatorname{sen} \alpha_{i j}=\operatorname{sen} 0^{\circ}=0$, se $i \neq j$, $\vec{\imath} \times \vec{\jmath}=\left(\operatorname{sen} \propto_{i j}\right) \vec{k}=\left(\operatorname{sen} 90^{\circ}\right) \vec{k}=\vec{k}, \log 0 \vec{A} \times \vec{B}$

$$
\sum_{i . j=1}^{3} A_{i} B_{j} \vec{\imath} \times \vec{\jmath}=
$$
}


Pois:

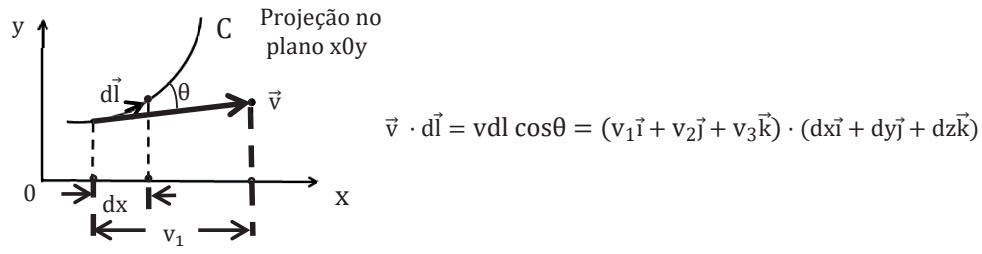

$$
\oint_{\mathrm{C}} \overrightarrow{\mathrm{E}} \cdot \mathrm{d} \vec{\ell}=\iint_{\mathrm{S}}(\vec{\nabla} \times \overrightarrow{\mathrm{E}}) \cdot \overrightarrow{\mathrm{n}} \mathrm{d} S, \quad \oint_{\mathrm{C}} \overrightarrow{\mathrm{E}} \cdot \mathrm{d} \vec{\ell}=-\frac{\mathrm{d}}{\mathrm{dt}} \phi_{\mathrm{B}} \quad \text { e, } \quad \phi_{\mathrm{B}}=\iint_{\mathrm{S}} \overrightarrow{\mathrm{B}} \cdot \overrightarrow{\mathrm{n}} \mathrm{d} S
$$

Terceira lei de Maxwell ou Faraday-Henry.

\subsubsection{Exemplo ilustrativo}

Calcular o trabalho da força de atração newtoniana de uma massa imóvel $m$ agindo sobre uma massa unitária se deslocando entre dois pontos (PISKOUNOV).

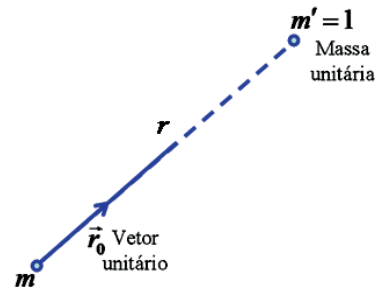

Força na massa unitária: $\vec{F}=-\frac{k m m^{\prime}}{r^{2}} \vec{r}_{0}=-\frac{k m}{r^{2}} \vec{r}_{0}$

Logo, $\vec{F}$ é o campo gravitacional: $\vec{g}=\frac{\vec{P}}{m^{\prime}} \quad m^{\prime}=1$

Componentes:

$=\left(A_{1} B_{2}-A_{2} B_{1}\right) \vec{k}+\left(A_{2} B_{3}-A_{3} B_{2}\right) \vec{\imath}+\left(A_{3} B_{1}-A_{1} B_{3}\right) \vec{\jmath}$, é um vetor perpendicular ao plano onde se situam os vetores $\vec{A}$ e $\vec{B}$. Isto é, uma matriz: $\vec{A} \times \vec{B}=\left|\begin{array}{ccc}\vec{\imath} & \vec{\jmath} & \vec{k} \\ A_{1} & A_{2} & A_{3} \\ B_{1} & B_{2} & B_{3}\end{array}\right|$ 


$$
X=-k m \frac{1}{r^{2}} \frac{x}{r} Y=-k m \frac{1}{r^{2}} \frac{y}{r} \quad Z=-k m \frac{1}{r^{2}} \frac{z}{r}
$$

$x / r \quad y / r \quad z / r \quad$ cossenos diretores

Trabalho para deslocar de $P_{1}$ a $P_{2}$ :

$$
A=-k m \int_{P_{1}}^{P_{2}} \frac{x d x+y d y+z d z}{r^{3}}=-k m \int_{P_{1}}^{P_{2}} \frac{r d r}{r^{3}}=k m \int_{P_{1}}^{P_{2}} d \frac{1}{r}
$$

Pois trabalho elementar dW é a força $\mathrm{F}$ agindo por um deslocamento dx, integrando:

$\mathrm{W}=\int \mathrm{Fdx}$, ver $1^{\mathrm{a}}$ página do Anexo 3.

$$
\begin{aligned}
& r^{2}=x^{2}+y^{2}+z^{2} \quad r d r=x d x+y d y+z d z,{ }^{25} \text { pois derivando } \mathrm{r}^{2}, \text { temos: } 2 \mathrm{r} \frac{\partial \mathrm{r}}{\partial \mathrm{x}}=2 \mathrm{x} \frac{\partial \mathrm{x}}{\partial \mathrm{x}} \text { e etc. } \\
& \therefore \frac{\partial \mathrm{r}}{\partial \mathrm{x}}=\frac{\mathrm{x}}{\mathrm{r}} \quad \therefore \partial \mathrm{r} / \partial \mathrm{y}=\mathrm{y} / \mathrm{r} \quad \text { e } \quad \partial \mathrm{r} / \partial \mathrm{z}=\mathrm{z} / \mathrm{r} \quad \text { (4) derivadas parciais }
\end{aligned}
$$

Por cálculo diferencial: $\mathrm{dr}=\frac{\partial \mathrm{r}}{\partial \mathrm{x}} \mathrm{dx}+\frac{\partial \mathrm{r}}{\partial \mathrm{y}} \mathrm{dy}+\frac{\partial \mathrm{r}}{\partial \mathrm{z}} \mathrm{dz}=\frac{\mathrm{x}}{\mathrm{r}} \mathrm{dx}+\frac{\mathrm{y}}{\mathrm{r}} \mathrm{dy}+\frac{\mathrm{z}}{\mathrm{r}} \mathrm{dz}=\frac{x d x+y d y+z d z}{\mathrm{r}}$, assim, temos a equação anterior.

$$
\text { Logo: } A=k m\left(\frac{1}{r_{2}}-\frac{1}{r_{1}}\right)
$$

$A$ é a diferença de potencial (trabalho por unidade de massa), ver Seção 4.7, Equação (1):

$$
u=\left(\frac{k m}{r}\right)
$$

é o potencial, então, derivando em relação às coordenadas:

$$
X=-\frac{\partial u}{\partial x} \quad Y=-\frac{\partial u}{\partial y} \quad Z=-\frac{\partial u}{\partial z} \text {, compare com as Equações (3) e (4). }{ }^{26}
$$

Este é o potencial gravitacional, assim denominado para distinguir-se do potencial eletromagnético, apresentado na Seção 5.13 .

${ }_{25}$ Além disso, pelo cálculo vetorial: $d A=\left(-\frac{k m r}{r^{3}} \vec{r}_{0}\right) \cdot\left(d r \vec{r}_{0}\right)=-\frac{k m}{r^{3}}(x \vec{\imath}+y \vec{\jmath}+z \vec{k}) \cdot(d x \vec{\imath}+d y \vec{\jmath}++d z \vec{k})=-k m \frac{r d r}{r^{3}}=-k m$ $\left(\frac{\mathrm{xdx}+\mathrm{ydy}+\mathrm{zdz}}{\mathrm{r}^{3}}\right)$, confirmando o resultado anterior.

${ }^{26}$ Conclui-se que o gradiente do potencial fornece o campo gravitacional com sinal trocado, $\overrightarrow{\mathrm{g}}=-\vec{\nabla} \mathrm{u}$. 
$\therefore A=u\left(P_{2}\right)-u\left(P_{1}\right) \quad$ A diferença de potencial gravitacional se manifesta como trabalho para deslocar a massa unitária $\mathrm{m}^{\prime}$ entre os pontos $P_{1}$ e $P_{2}$. Independe do caminho percorrido, e só depende das coordenadas dos pontos.

\subsubsection{Velocidade angular em torno de 0}

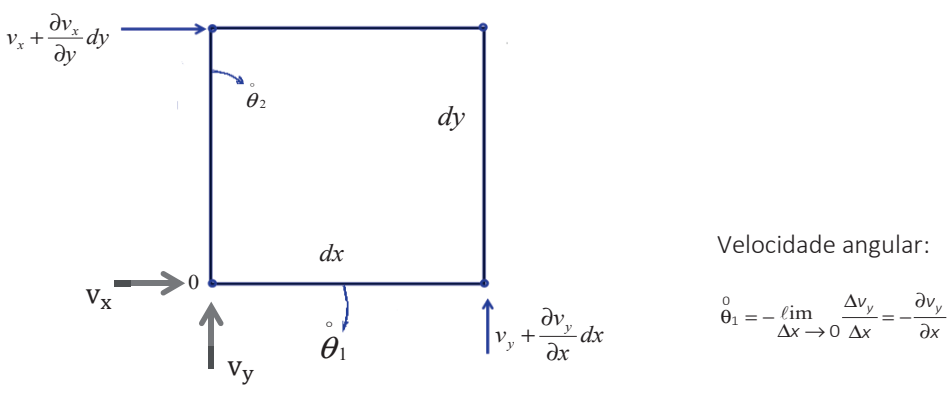

${\stackrel{0}{\theta_{1}}}_{1}=-\frac{\partial v_{y}}{\partial x}=-\frac{\left[v_{y}+\frac{\partial v_{y}}{\partial x} d x\right]-v_{y}}{d x}=-\frac{\partial}{\partial x}\left(\frac{\partial y}{\partial t}\right) \Rightarrow \theta_{1}=\partial y / \partial x$, ângulo

Velocidade angular é ângulo na unidade de tempo. ${ }^{27}$

$\stackrel{0}{\theta_{2}}=+\frac{\partial v_{x}}{\partial y}=+\frac{\left[v_{x}+\frac{\partial v_{x}}{\partial y} d y\right]-v_{x}}{d y}=\frac{\partial}{\partial y}\left(\frac{\partial x}{\partial t}\right) \Rightarrow \theta_{2}=\partial x / \partial y$

Velocidade angular média em torno do eixo $z: \omega_{\mathrm{z}}=\frac{1}{2}\left(\dot{\theta}_{1}+\dot{\theta}_{2}\right)$

$$
\omega_{z}=\frac{1}{2}\left(\frac{\partial v_{x}}{\partial y}-\frac{\partial v_{y}}{\partial x}\right)=\frac{1}{2}(\operatorname{rot} \vec{v})_{z}
$$

Ver Seção 5.15, sobre o teorema de Stokes, Equação (1), em que deduzimos o $(\operatorname{rot} \vec{v})_{\mathrm{z}}$ através da circulação:

$$
\Gamma=\oint_{t} \overrightarrow{\mathrm{v}} \cdot \mathrm{d} \vec{\ell}
$$

isto é, $\quad(\operatorname{rot} \overrightarrow{\mathrm{v}})_{\mathrm{z}}=\frac{\partial \mathrm{v}_{\mathrm{y}}}{\partial \mathrm{x}}-\frac{\partial \mathrm{v}_{\mathrm{x}}}{\partial \mathrm{y}}=\frac{\mathrm{d} \Gamma}{\mathrm{dS}} 28$

A figura ao lado da Equação (1) é uma representação gráfica do rotacional no plano bidimensional xy. Para uma interpretação gráfica do rotacional no espaço tridimensional, ver figura ao lado da Equação

27 Ver Seção 2.1.2, Pêndulo simples, equação 1.

${ }^{28}$ Apresentamos aqui a obtenção do rotacional bidimensional para um campo de velocidades. Contudo, essa forma é válida para qualquer variável, como poderá ser visto a seguir. O conceito de campo foi introduzido na Seção 5.3. 
(3) na Seção 5.16, em que a variação da relação entre a circulação $\Gamma$ representada pelo contorno do orifício $\mathrm{L}$ e a superfície $\mathrm{S}$ da bexiga estão indicadas. A definição matemática do rotacional tridimensional encontra-se na Equação (5a) da Seção 5.16.

O rotacional significa que, além da componente axial, existe uma componente tangencial provocando circulação no plano perpendicular ao eixo considerado, isto é, provocando turbilhão no fluxo vetorial, ou seja, uma rotação.

Escrevendo a lei de Faraday na forma diferencial:

$$
\begin{aligned}
& \left(\frac{\partial E_{2}}{\partial x}-\frac{\partial E_{1}}{\partial y}\right) \vec{e}_{3}+\left(\frac{\partial E_{3}}{\partial y}-\frac{\partial E_{2}}{\partial z}\right) \vec{e}_{1}+\left(\frac{\partial E_{1}}{\partial z}-\frac{\partial E_{3}}{\partial x}\right) \vec{e}_{2}=-\left(\frac{\partial B_{1}}{\partial t} \vec{e}_{1}+\frac{\partial B_{2}}{\partial t} \vec{e}_{2}+\frac{\partial B_{3}}{\partial t} \vec{e}_{3}\right)=\operatorname{rot} \vec{E} \\
& \text { Ou: } \quad\left(\frac{\partial E_{j}}{\partial x_{i}}-\frac{\partial E_{i}}{\partial y_{j}}\right) \vec{e}_{k}=-\frac{\partial B_{i}}{\partial t} \vec{e}_{i}=(\operatorname{rot} \vec{E})_{i} \vec{e}_{i}, \text { e } \therefore \vec{\nabla} \times \vec{E}=-\frac{\partial}{\partial t} \vec{B} \\
& \quad i, j, k=1,2,3
\end{aligned}
$$

Como visto na Equação (2) da Seção 5.15.

\subsection{LEI DE AMPÈRE-MAXWELL}

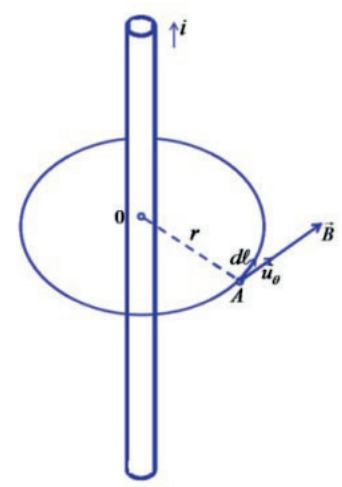

Com a corrente $i$ surge um campo de indução magnética $\vec{B}$ em volta do condutor, de valor: $\vec{B}=\frac{\mu_{0} i}{2 \pi r} \vec{u}_{\theta}, \vec{u}_{\theta}$ vetor unitário. $\vec{B}$ é diretamente proporcional a $i$ e inversamente proporcional a $r$.

Calculemos a circulação magnética:

$\Gamma=\oint_{\mathrm{C}} \overrightarrow{\mathrm{B}} \cdot \mathrm{d} \vec{\ell}=\mathrm{B} \int \mathrm{d} \ell=\left(\frac{\mu_{0} \mathrm{i}}{2 \pi \mathrm{r}}\right) 2 \pi \mathrm{r}=\mu_{0} \mathrm{i}$ 
$\mu_{0}=$ constante de permeabilidade, ver Seções 5.23 e 5.24 .

Trata-se da lei de Ampère (1755-1836).

James Clerk Maxwell (1831-1879) percebeu que Faraday foi o primeiro a compreender corretamente os fenômenos elétricos e magnéticos. O trabalho de Faraday fora experimental, e Maxwell se propôs a expor matematicamente os conhecimentos de eletricidade e magnetismo da época, reunindo suas conclusões no Tratado de Eletricidade e Magnetismo, publicado em 1873. (0)

Seja o fluxo de corrente através de uma superfície de controle SC (ver Seção 5.9).

A corrente $i$ será: $i=\oint_{S} \rho \vec{v} \cdot \vec{n} d S \quad 29 \quad i=-\frac{d q}{d t}$

Nesse caso, a superfície é fechada.

$i$ é o fluxo da carga por unidade de tempo.

O fluxo $\varepsilon_{0} \phi_{\mathrm{e}}$ saindo da superfície é positivo, pois $\vec{v} \cdot \vec{n}=v \cos \theta, \theta=$ angulo entre $\vec{v}$ e $\vec{n}, \theta<90^{\circ}$, $\log 0, \cos \theta>0$. Porém, o fluxo da corrente saindo da superfície fechada significa diminuição da carga no $V C$. Então, $d q / d t<0$, pois $d q<0$ e $d t>0$. (ver figura da Seção 5.9).

Logo, teremos: $-\frac{d q}{d t}=\oint_{S} \rho \vec{v} \cdot \vec{n} d S=-\varepsilon_{0} \frac{\mathrm{d} \phi_{\mathrm{e}}}{\mathrm{dt}}$

ver Seção 5.10, Equação (0).

Porém,

$q=\varepsilon_{0} \oint_{S} \vec{E} \cdot \vec{n} d S$, portanto: $\frac{\mathrm{dq}}{\mathrm{dt}}=\varepsilon_{0} \frac{\mathrm{d}}{\mathrm{dt}} \int_{S} \overrightarrow{\mathrm{E}} \cdot \overrightarrow{\mathrm{n}} \mathrm{d} \overrightarrow{\mathrm{S}}$

(pela lei de Gauss para eletricidade, Seção 5.10, e teorema de Gauss-

$q=\int_{V} \rho d V=\varepsilon_{0} \int_{V} \vec{\nabla} \cdot \vec{E} d V=\varepsilon_{0} \oint_{S} \vec{E} \cdot \vec{n} d S=\varepsilon_{0} \phi_{e}$

Ostrogradsky)

Substituindo em 1a: $\oint_{S} \rho \vec{v} \cdot \vec{n} d S+\varepsilon_{0} \frac{d}{d t} \oint_{S} \vec{E} \cdot \vec{n} d S=0$

É o principio da conservação da carga em uma $S C$.

${ }^{29}$ Ver Seção 5.9, Equação (1), $\oint_{S} \rho \vec{v} \cdot \vec{n} d S=-\frac{\partial}{\partial t} \int_{V} \rho d V=-\frac{\partial}{\partial t} \int_{V} \frac{d q}{d V} d V=-\frac{d q}{d t}=i, \rho=\frac{\mathrm{dq}}{\mathrm{dV}}$. 
Se os campos forem estáticos, a integral $\oint_{S} \vec{E} \cdot \vec{n} d S$ não depende do tempo, e sua derivada em relação ao tempo é nula. Portanto: $\oint_{S} \rho \vec{v} \cdot \vec{n} d S=0$ para campos estáticos, a carga q é constante no tempo através da superfície de controle $S C$. Como o fluxo elétrico é: $\phi_{\mathrm{e}}=\mathrm{q} / \varepsilon_{0}$, ver Seção 5.10, o fluxo elétrico $\phi_{\mathrm{e}}$ na $S C$ é constante.

Maxwell sugeriu uma modificação da lei de Ampère, que foi deduzida em condições estáticas.

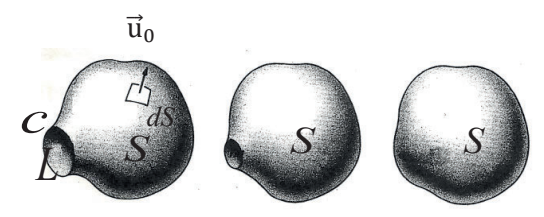

É aplicada a uma superfície $S$ limitada pela linha $c$.

$$
\oint_{c} \vec{B} \cdot d \vec{\ell}=\int_{s} \operatorname{rot} \vec{B} \cdot \vec{u}_{0} d S
$$

pelo teorema de Stokes.

Pode ser expressa como:

$$
\begin{aligned}
& \oint_{C} \vec{B} \cdot \overrightarrow{d \ell}=\mu_{0} \int_{S} \rho \vec{v} \cdot \vec{n} \mathrm{~d} S=\mu_{0} \mathrm{i}[\operatorname{ver} \text { Equação (1)]. } \\
& \overrightarrow{\mathrm{u}}_{0}=\text { vetor unitário } \perp \text { a dS }
\end{aligned}
$$

Se a linha se contrai a um ponto, a superfície se torna fechada e, nesse caso, $\oint_{S} \rho \vec{v} \cdot \vec{n} d S=0,{ }^{30}$ que concorda com o principio de conservação da carga como vimos. Contudo, isso é para campo estático, não sendo válida quando dependente do tempo. A modificação sugerida se torna: $\oint_{c} \vec{B} \cdot d \vec{\ell}=\mu_{0} \int_{S} \rho \vec{v} \cdot \vec{n} d S+\varepsilon_{0} \mu_{0} \frac{d}{d t} \int_{S} \vec{E} \cdot \vec{n} d S \quad$ ou

$\oint_{c} \vec{B} \cdot \overrightarrow{d \ell}=\mu_{0} i+\varepsilon_{0} \mu_{0} \frac{d}{d t} \int_{S} \vec{E} \cdot \vec{n} d S ;$ conforme equação 2 a, nesse caso, não será nulo

É a lei de Ampère-Maxwell ou $4^{a}$ Equação de Maxwell.

Ela se reduz à lei de Ampère para campos estáticos, pois o último termo é nulo, quando a linha se reduz a um ponto e a superfície $S$ se torna fechada. A modificação de Maxwell foi mais pela necessidade

\footnotetext{
${ }^{30}$ Pois dl fica nulo.
} 
de consistência matemática do que por experiências. As experiências que comprovaram sua modificação apareceram alguns anos mais tarde.

O termo correspondente à "corrente elétrica", que seria "corrente magnética", não aparece, pois não existem polos magnéticos livres na natureza, isto é, não há cargas magnéticas. Estamos nos referindo à lei de Faraday-Henry: $\oint_{c} \vec{E} \cdot d \ell=-\frac{d \phi_{B}}{d t}, 31$

ver final da Seção 5.14.1, Equação (2).

Aplicando o teorema de Stokes na lei de Ampère-Maxwell: transforma integral de linha em integral de superfície, ver no final da Seção 5.15, Equação (1a): ${ }^{32}$

$$
\oint_{c} \vec{B} \cdot \overrightarrow{d \ell}=\int_{S} \operatorname{rot} \vec{B} \cdot \vec{n} d S=\mu_{0} \int_{S} \rho \vec{v} \cdot \vec{n} d S+\varepsilon_{0} \mu_{0} \frac{d}{d t} \int_{S} \vec{E} \cdot \vec{n} d S=f
$$

Tendo em vista que:

$$
\overrightarrow{\mathrm{n}} \cdot \operatorname{rot} \overrightarrow{\mathrm{v}}=\lim _{\Delta S \rightarrow 0} \frac{\Delta \Gamma}{\Delta S}=\lim _{\Delta S \rightarrow 0} \frac{\oint_{c} \vec{v} \cdot \overrightarrow{d \ell}}{\Delta S} \quad \text { (5a) } \quad \Rightarrow \oint_{\mathrm{C}} \overrightarrow{\mathrm{v}} \cdot \mathrm{d} \vec{\ell}=\int_{\mathrm{S}} \operatorname{rot} \overrightarrow{\mathrm{v}} \cdot \overrightarrow{\mathrm{n}} \mathrm{d} S
$$

e levando ao limite da mesma forma que: $\operatorname{div} \vec{v}=\lim _{\Delta V \rightarrow 0} \frac{\oint_{S} \vec{v} \cdot \vec{n} d S}{\Delta V} \quad$ (Ver Seção 5.7)

Obtemos: $\operatorname{rot} \vec{B}=\mu_{0} \rho \vec{v}+\varepsilon_{0} \mu_{0} \frac{d \vec{E}}{d t}(5)$

Forma diferencial da lei de Ampère- Maxwell e $4^{\mathrm{a}}$ lei de Maxwell.

Obtida da Equação (4), por diferenciação, isto é, chamando (4) de $f$, achamos a quantidade elementar $d f$ da função $f$, ou melhor, derivamos em relação a $\vec{n} S$, procedemos à operação inversa da integração, como visto na Seção 5.9, Equação (2).

\subsubsection{Exemplo ilustrativo}

Seja um campo elétrico uniforme $\overrightarrow{\mathrm{E}}$ em uma região cilíndrica do espaço. Por exemplo, um condensador com armaduras circulares planas e paralelas, de raio $\mathrm{R}$, sendo carregado como na Figura a seguir. Deduzir o campo magnético B em pontos internos do capacitor: $r \leqq R$. Não existe corrente entre as placas, então $\mathrm{i}=0$ e $\mu_{0} \mathrm{i}=0$.

\footnotetext{
${ }^{31}$ Compare com: $\oint \vec{B} \cdot d \vec{\ell}=\mu_{0} i+\varepsilon_{0} \mu_{0} \frac{d \phi_{E}}{d t} \quad$ (ver início da Seção 5.10). O termo $\mu_{0} i$ é o da corrente elétrica.

${ }^{32}$ Recorde-se que: $\vec{\nabla} \times \overrightarrow{\mathrm{B}}=\operatorname{rot} \overrightarrow{\mathrm{B}}$.
} 

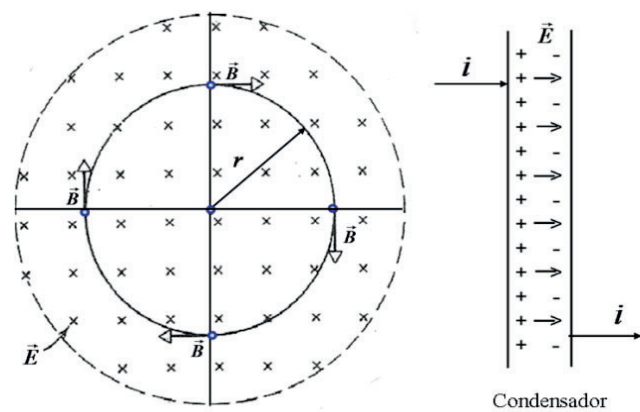

A intensidade do campo elétrico $\vec{E}$ aumenta uniformemente: $d E / d t$ constante e positivamente. A carga das armaduras também aumenta uniformemente. Uma corrente flui para a armadura positiva, e outra igual sai da armadura negativa.

O campo elétrico variável cria um campo magnético induzido, isto é, a indução magnética $\vec{B}$.

Isso pode ser expresso como:

$\oint_{c} \vec{B} \cdot \overrightarrow{d \ell}=\mu_{0} \varepsilon_{0} \frac{d \phi_{e}}{d t}$, combinando as Equações (1), (2) e (3),,$^{33} \phi_{e}=\int_{S} \vec{E} \cdot \vec{n} d S$, ver Equação (1a) da Seção 5.10 .

Compare com a última figura da Seção 5.14, é a contrapartida da lei da indução de Faraday. Repare que $\vec{B}$ está em sentido contrário a $\vec{E}$, por isso a troca de sinais.

Circulação de $\mathrm{B}=\mu_{0} \varepsilon_{0}$ (variação temporal do fluxo elétrico $\left.\phi_{\mathrm{e}}\right) \Rightarrow$

$\Rightarrow(B)(2 \pi r)=\mu_{0} \varepsilon_{0} \pi r^{2} \frac{d E}{d t}, \vec{E} \cdot \vec{n}$ constante na superfície $S$ em determinado instante $t$.

Explicitando B: $B=\frac{1}{2} \mu_{0} \varepsilon_{0} r \frac{d E}{d t}$, para $r \leqq R$.

$33 \oint_{C} \vec{B} \cdot d \vec{\ell}=\mu_{0} i=-\mu_{0} \frac{d q}{d t} \quad$ por $1 a:-\frac{d q}{d t}=\varepsilon_{0} \phi_{8} / d t \quad \therefore \oint_{C} \vec{B} \cdot d \vec{\ell}=\mu_{0} \varepsilon_{0} \frac{d}{d t} \oint_{\delta} \vec{E} \cdot \vec{n} d S$

Recorde-se que: $\mathrm{i}=-\frac{\mathrm{dq}}{\mathrm{dt}}$ e $\quad \phi_{\mathrm{e}}=\mathrm{q} / \varepsilon_{0}$. 


\subsection{ENERGIA POTENCIAL}

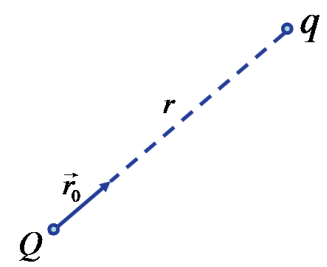

Referindo-se à Seção 5.15, exemplo ilustrativo, se substituirmos as massas m e m' pelas cargas Q e $q$, a força será: $\vec{F}=-\frac{1}{4 \pi \varepsilon_{0}} \frac{Q q}{r^{2}} \vec{r}_{0}{ }^{34}$

$\overrightarrow{\mathrm{r}}_{0}$ vetor unitário supondo cargas de sinais contrários.

Campo elétrico: $\vec{E}=\frac{\vec{F}}{q}$

Força para carga unitária: $q=1$

Componentes:

$$
X=-\frac{Q}{4 \pi \varepsilon_{0}} \frac{1}{r^{2}} \frac{x}{r} \quad Y=-\frac{Q}{4 \pi \varepsilon_{0}} \frac{1}{r^{2}} \frac{y}{r} \quad Z=-\frac{Q}{4 \pi \varepsilon_{0}} \frac{1}{r^{2}} \frac{z}{r}
$$

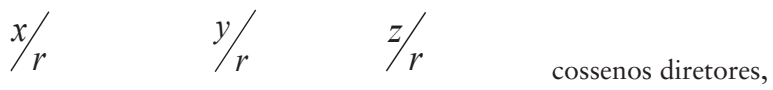

Trabalho para deslocar de $P_{1}$ a $P_{2}: \mathrm{W}=\mathrm{E}_{\mathrm{p}}=\int_{\mathrm{P}_{1}}^{\mathrm{P}_{2}} \overrightarrow{\mathrm{F}} \cdot \mathrm{d} \overrightarrow{\mathrm{r}}$

$E_{p}=-\frac{Q q}{4 \pi \varepsilon_{0}} \int_{P_{1}}^{P_{2}} \frac{x d x+y d y+z d z}{r^{3}}=-\frac{Q q}{4 \pi \varepsilon_{0}} \int_{P_{1}}^{P_{2}} \frac{r d r}{r^{3}}=\frac{Q q}{4 \pi \varepsilon_{0}} \int_{P_{1}}^{P_{2}} d \frac{1}{r}$

$r^{2}=x^{2}+y^{2}+z^{2} \quad r d r=x d x+y d y+z d z \quad \therefore \frac{\partial \mathrm{r}}{\partial \mathrm{x}}=\frac{\mathrm{x}}{\mathrm{r}} \quad \frac{\partial \mathrm{r}}{\partial \mathrm{y}}=\frac{\mathrm{y}}{\mathrm{r}} \mathrm{e} \frac{\partial \mathrm{r}}{\partial \mathrm{z}}=\frac{\mathrm{z}}{\mathrm{r}} 35$ derivadas parciais

$\therefore \mathrm{E}_{\mathrm{p}}=\frac{\mathrm{Qq}}{4 \pi \varepsilon_{0}}\left(\frac{1}{\mathrm{r}_{2}}-\frac{1}{\mathrm{r}_{1}}\right)$, Energia potencial elétrica.

Sobre o trabalho, ver exemplo ilustrativo da Seção 5.15.1

4 Nesse caso, nos restringimos à força entre cargas elétricas.

35 Ver detalhes no exemplo ilustrativo da Seção 5.15.1, Equação $42 \mathrm{r} \partial \mathrm{r} / \partial \mathrm{x}=2 \mathrm{x} \partial \mathrm{x} / \partial \mathrm{x}$. 


\subsubsection{Potencial elétrico}

Ainda se restringindo à força entre cargas elétricas, se tomarmos $E_{p}$ e dividirmos por $q$ teremos a diferença de potencial: ${ }^{36}$

$$
\Delta V=\frac{E_{p}}{q}=\frac{Q}{4 \pi \varepsilon_{0}}\left(\frac{1}{r_{2}}-\frac{1}{r_{1}}\right)_{(0)^{37}}
$$

Chamando $V$ de potencial: $V=\frac{Q}{4 \pi \varepsilon_{0}} \frac{1}{r}(1)$

Então: $X=-\frac{\partial V}{\partial x} \quad Y=-\frac{\partial V}{\partial y} \quad Z=-\frac{\partial V}{\partial z}$, compare com as grandezas mencionadas na seção 5.17, pois $\frac{\partial \mathrm{V}}{\partial \mathrm{x}}=-\frac{\mathrm{Q}}{4 \pi \varepsilon_{0}} \frac{1}{\mathrm{r}^{2}} \frac{\partial \mathrm{r}}{\partial \mathrm{x}} \mathrm{e} \frac{\partial \mathrm{r}}{\partial \mathrm{x}}=\frac{\mathrm{x}}{\mathrm{r}}$ etc. Ver Seção 5.17, Equação (1), X, Y e Z são as componentes de $\overrightarrow{\mathrm{E}}$.

$$
\Delta V=V\left(P_{2}\right)-V\left(P_{1}\right)
$$

A diferença de potencial se manifesta como trabalho para deslocar a carga unitária q entre os pontos $P_{1}$ e $P_{2}$. Independe do caminho percorrido, e depende somente das coordenadas dos pontos $(\mathrm{x}, \mathrm{y}, \mathrm{z})$.

Pelo visto anteriormente, deduzimos que:

$\vec{E}=-\operatorname{grad} V$

Ou $\vec{E}=-\vec{\nabla} V$ (ver Seção anterior, idem ao já mencionado; $\mathrm{X}, \mathrm{Y}, \mathrm{Z}$ componentes de $\overrightarrow{\mathrm{E}}$ ).

Sendo $\vec{\nabla}=\frac{\partial}{\partial x} \vec{i}+\frac{\partial}{\partial y} \vec{j}+\frac{\partial}{\partial z} \vec{k}$

$\overrightarrow{\mathrm{E}}=\overrightarrow{\mathrm{F}} / \mathrm{q}=-$ cte $\frac{\mathrm{Q}}{\mathrm{r}^{2}} \overrightarrow{\mathrm{r}}_{0}$, porém, $V=$ cte $\mathrm{Q} / \mathrm{r}, \operatorname{logo}: \vec{\nabla} \mathrm{V}=-$ constante $\frac{\mathrm{Q}}{\mathrm{r}^{2}} \overrightarrow{\mathrm{r}}_{0}=\overrightarrow{\mathrm{E}}$, ver Equação (1a).

O campo elétrico $\vec{E}$ é igual ao gradiente do potencial $V$ com sinal trocado, ou seja, o vetor campo elétrico é perpendicular às superfícies equipotenciais e, como ele é negativo, aponta no sentido dos potenciais decrescentes.

36 A parcela devida à energia magnética será tratada na Seção 5.21.

37 Recordemos a integral definida: $\int_{a}^{b} f(x) d x=F(b)-F(a)=A$ (ver equação de $E_{p}$ na Seção 5.17).

Em que: $\int f(x) d x=F(x)+$ cte e $A=$ Área sob a curva $y=f(x)$, entre: $a \leqq x \leqq b$.

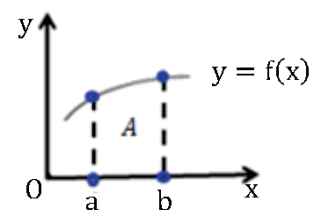


A unidade usada para o potencial é o volt $(V)$ e, para o campo elétrico, volt $/ \mathrm{m}$ ou newton $/$ coulomb . Lembremos que: $\vec{E}=\vec{F} / q \cdot \cdot^{38}$

Sejam duas superfícies planas e paralelas com potenciais $V_{1}$ e $V_{2}$. Essas superfícies são equipotenciais, isto é, seus pontos estão a um mesmo potencial, respectivamente $V_{1}$ e $V_{2}$.

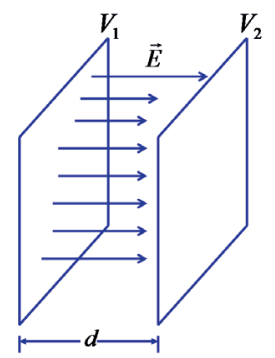

$$
\begin{aligned}
& E=-\frac{V_{2}-V_{1}}{d}=\frac{V_{1}-V_{2}}{d} \\
& \Delta V=V_{2}-V_{1}
\end{aligned}
$$

Diferença de potencial $\therefore \mathrm{E}=-\frac{\Delta \mathrm{V}}{\mathrm{d}}$

\subsection{CAPACIDADE ELÉTRICA, CAPACITORES}

O potencial elétrico na superfície esférica equipotencial de raio $r$, tendo uma carga $Q$ concentrada em seu centro, é, como visto na Seção 5.17.1, Equação (1): $V=\left|\frac{Q}{4 \pi \varepsilon_{0}} \frac{1}{r}\right|$, isto é, em módulo.

Se a carga estiver envolta por um dielétrico, um material isolante, substituiremos $\varepsilon_{0}$ por $\varepsilon$ : $V=\left|\frac{Q}{4 \pi \varepsilon} \frac{1}{r}\right|$, em que $\varepsilon$ é a permissividade do isolante, e $\varepsilon_{0}$, permissividade no vácuo.

A relação $Q / V$ para a esfera é constante e igual a $4 \pi \varepsilon r$, e é denominada "Capacidade": $C=\frac{Q}{V}=4 \pi \varepsilon r$.

${ }^{38} \mathrm{O}$ volt é joule/coulomb, pois potencial é energia/carga. Recordar Seção 5.6, "Linhas de força". Como $\overrightarrow{\mathrm{E}}=\overrightarrow{\mathrm{F}} / \mathrm{q}, \mathrm{dE} \mathrm{E}_{\mathrm{p}}=\overrightarrow{\mathrm{F}}_{\mathrm{i}}$. $d \vec{x}_{i}, d V=d E_{p} / q, \vec{E}=-\overrightarrow{\nabla V}=-\partial V / \partial x_{i} \vec{e}_{i}=-\frac{\partial}{\partial x_{i}}\left(E_{p} / q\right) \vec{e}_{i}=\vec{F} / q$ 
A capacidade é expressa em farad, homenagem a Michael Faraday.

O farad é definido como a capacidade de um condutor que, recebendo uma carga de 1 Coulomb, fica com um potencial de 1 Volt.

O conceito de capacidade elétrica pode ser estendido para um sistema de condutores. Sejam os dois condutores na Figura com cargas, respectivamente, $+Q$ e $-Q$, a capacidade de um sistema é definida como: $C=\frac{Q}{V_{1}-V_{2}}=\frac{Q}{\Delta V} \quad V_{1}-V_{2}=E d$, ver equação $1 \mathrm{~b}$ na secção 5.17 .1

Esse arranjo é chamado de capacitor.

Os capacitores ou condensadores servem para armazenar energia elétrica na forma de energia potencial, e acumulam carga. Exemplos de aplicação, dos condensadores temos: desfibrilador ventricular, flash de máquina fotográfica, circuitos de sintonização de transmissores e receptores de rádio e televisão, bancos de memória de computadores, entre outros.

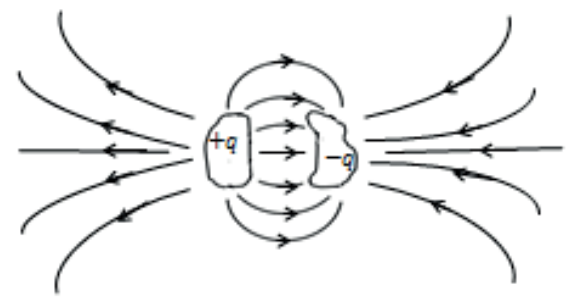

Elementos básicos: dois condutores isolados de formato arbitrário. Os condutores são as "placas". O mais convencional é o de placas paralelas já visto na Figura da Seção 5.17.1, mas também podem ser cilíndricos. Quando carregados, suas placas adquirem cargas iguais, mas de sinais opostos. Para carregálo, colocamos seus terminais em contato com uma bateria.

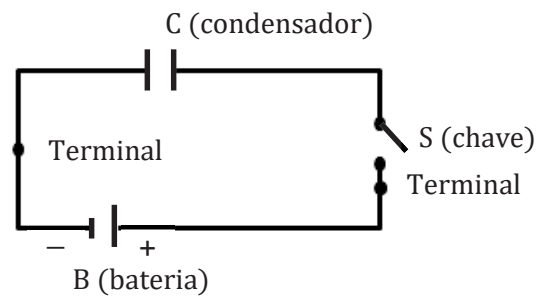

A garrafa de Leyden é o condensador mais antigo. Seu nome vem da cidade onde foi inventada ${ }^{39}$ Foi inventada em 1746. A experiência de Franklin foi posterior. Benjamin Franklin (1706-1790) demonstrou

\footnotetext{
39 Cidade na Holanda, sede de uma Universidade importante.
} 
que quando um condensador é carregado, não só as armaduras ficam eletrizadas, mas também o dielétrico (isolante).

Conforme a Figura a seguir, na garrafa de Leyden, o dielétrico é um vaso cônico de vidro A, a armadura externa é um vaso metálico B que se adapta externamente a A; a armadura interna é um tronco de cone maciço $\mathrm{C}$ que se adapta internamente a $\mathrm{A}$ e com uma haste $\mathrm{H}$ através da qual é eletrizado. Em $\mathrm{D}$ indica-se o condensador montado, o qual é carregado. Ao desmontar as armaduras, o contato com as mãos as descarregam. Montando-se novamente, as armaduras ficam outra vez carregadas, indicando que o dielétrico A estava eletrizado, carregando as armaduras depois da $2^{\mathrm{a}}$ montagem.

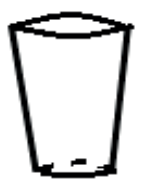

A

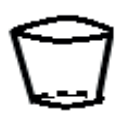

$\mathrm{B}$

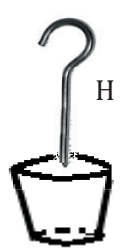

C

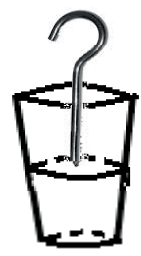

$\mathrm{D}$

Pode-se fazer analogia entre um capacitor carregado com uma carga q e um recipiente de paredes rígidas de volume $\mathrm{V}$, contendo massa $\mathrm{m}$ de gás perfeito. A pressão $\mathrm{p}$ do gás (para uma temperatura dada) é diretamente proporcional a $\mathrm{m}$, de acordo com as leis dos gases perfeitos: $\mathrm{m}=\left(\frac{\mathrm{V}}{\mathrm{RT}}\right) \mathrm{p}$. Para o capacitor teremos: $q=(C) V$. Comparando, vemos que a capacitância $\mathrm{C}$ é o análogo do volume $\mathrm{V}$ do recipiente (supondo $\mathrm{T}$ constante) e à carga $\mathrm{q}$ corresponde a massa $\mathrm{m}$.

Observe que referimo-nos à carga de um capacitor como sendo q, o valor absoluto da carga de uma das armaduras. No entanto, sua carga líquida é nula.

Considera-se que o condensador acumula energia elétrica na forma de energia potencial no campo elétrico existente entre as placas do capacitor. São capazes de confinar os campos elétricos em volumes pequenos, sendo dispositivos acumuladores de energia. Nos síncrotons (cíclotron acelerador de elétrons), a energia acumulada em capacitores durante um longo tempo é liberada de modo intermitente para aceleração dos elétrons, por meio da descarga dos capacitores, em um tempo muito mais curto.

$\mathrm{Na}$ física dos plasmas também se faz uso dos capacitores. 


\subsection{ENERGIA DO CAMPO ELÉTRICO}

Tendo em vista o parágrafo anterior, ao carregar um condutor ou capacitor, gastamos energia ao trazer mais carga, e realizamos trabalho para superar a repulsão da carga existente, se houver.

Seja um condutor ou capacitor de capacidade $C$ com carga $q$. Seu potencial é $V=q / C \cdot{ }^{40}$ Adicionando $d q$, trazendo do infinito, o trabalho efetuado é $d W=V d q$, igual ao acréscimo $d E_{e}$ na energia do condutor.

Então:

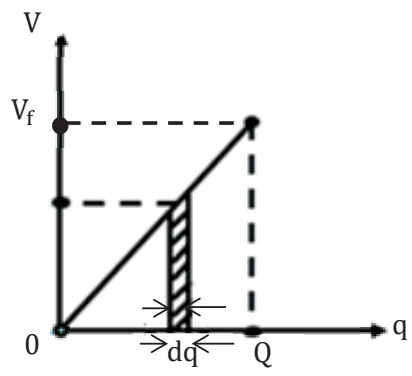

$\mathrm{dE}_{\mathrm{e}}=\frac{\mathrm{qdq}}{\mathrm{C}}$ Integrando de 0 (zero) até a carga total $Q$.

$$
\mathrm{E}_{\mathrm{e}}=\frac{1}{\mathrm{C}} \int_{0}^{\mathrm{Q}} \mathrm{qdq}=\frac{1}{2} \frac{\mathrm{Q}^{2}}{\mathrm{C}}
$$

$$
\mathrm{E}_{\mathrm{e}}=\int_{0}^{\mathrm{Q}} \mathrm{Vdq} \text {, nota-se que: } \mathrm{E}_{\mathrm{e}}=\frac{1}{2} \mathrm{~V}_{\mathrm{f}} \mathrm{Q}
$$

Recorde que: $\quad \mathrm{V}=\mathrm{E}_{\mathrm{p}} / \mathrm{Q}$, ver Seção 5.17.1, Equação (0).

É a área de um triângulo, ver figura, $\mathrm{V}_{\mathrm{f}}$ seria a ddp final entre os terminais do condensador, quando sua carga for a carga total $Q$ e a ddp inicial fosse nula.

\footnotetext{
${ }^{40}$ No caso de um capacitor, V será a diferença de potencial, ddp, entre as placas.
} 


\subsection{AUTOINDUÇÃo}

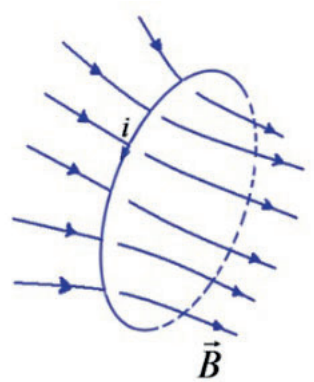

Seja um circuito percorrido pela corrente $i$. Conforme a lei de Ampère, Seção 5.16, Equação (1), a corrente produz um campo magnético que, em cada ponto, é proporcional a i. Esse fluxo $\phi_{i}$ é, portanto, proporcional à corrente $i: \phi_{i}=L i$. $^{41}$

$L$ depende da forma geométrica do condutor e é chamada "autoindutância" do circuito.

É expresso em uma unidade denominada henry, em homenagem a Joseph Henry (1797-1878), contemporâneo de Faraday.

Se houver variação da corrente i com o tempo, o fluxo magnético $\phi_{i}$ também varia e, conforme a Lei de Faraday-Henry [ver Seção 5.14, Equação (1)], uma força eletromotriz fem, isto é, uma diferença de potencial $\Delta V_{L}$, é induzida no circuito. É chamada de "autoindução": $\Delta V_{L}=-\frac{d \phi_{i}}{d t}=-L \frac{d i}{d t}$

O sinal negativo indica que $\Delta V_{L}$ se opõe à variação da corrente, isto é, para dt positivo, di será negativo.

A equação é válida para o circuito rígido e, portanto, para $L$ constante. Se o circuito for variável, $L$ não será constante e:

$$
\Delta V_{L}=-\frac{d}{d t}(L i)
$$

\footnotetext{
${ }^{41}$ A relação entre L e a indução $\vec{B}$ e o fluxo magnético $\phi_{\mathrm{B}}$ será apresentada na Seção 5.26 , ao tratarmos do vetor de Poynting, no caso de um solenoide.
} 


\subsection{ENERGIA DO CAMPO MAGNÉTICO}

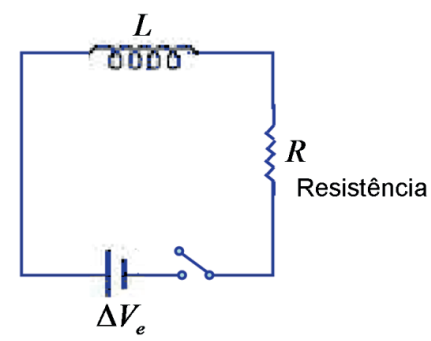

Seja o circuito em que é aplicada uma fem $\Delta V_{e}{ }^{42}$ com o circuito inicialmente desligado. Ao se ligar o circuito, devido à indutância $L$, que atua se opondo à corrente, como se fosse para vencer uma inércia, a corrente não atinge o valor instantaneamente, mas, aos poucos, o valor $\Delta V_{e} / R$. A resistência $\mathrm{R}^{43}$ representa as perdas de energia do circuito que se transformam em energia térmica, ou seja, calor, pelo efeito Joule.

Claro que, pela lei da conservação da energia, a diferença de potencial em um nó será:

$$
\Delta \mathrm{V}_{\mathrm{e}}-\mathrm{L} \frac{\mathrm{di}}{\mathrm{dt}}-\mathrm{Ri}=0
$$

Ri é a queda de tensão devido às perdas de energia. Daqui se deduz a lei de Ohm, válida para metais condutores. Essa queda de tensão é uma função linear com a corrente $\mathrm{i}$, sendo $R$ uma constante que depende do condutor, e sua geometria, é medida em ohms.

$$
R i=\Delta V_{e}-L \frac{d i}{d t} \quad \text { Logo: } \Delta V_{e} i=R i^{2}+L i \frac{d i}{d t}
$$

O $1^{\circ}$ termo representa $\Delta V_{e}^{d q} / d t$, que é a taxa da energia por unidade de tempo da fem fornecida ao circuito (ver Seção 5.13), diferença de potencial multiplicado pela corrente.

O $2^{\circ}$ termo representa a taxa de produção de calor no circuito. Para uma explicação física desse termo, ver Seção 5.26, Equação (5.8a 2).

$\mathrm{O} 3^{\circ}$ termo representa a energia por unidade de tempo para formar a corrente ou estabelecer seu campo magnético associado.

${ }^{42}$ Um dispositivo capaz de manter uma diferença de potencial $\Delta V_{e}$ [ver Seção 5.17.1, Equação (2)] entre seus terminais, é denominado fonte de força eletromotriz fem, por exemplo: bateria, pilha ou gerador.

${ }^{43} \mathrm{Em}$ 1827, Georg Ohm estabeleceu uma analogia entre um circuito hidráulico e um circuito elétrico: a vazão corresponderia à corrente, ao desnível, a diferença de potencial e a oposição ao fluxo à resistência R. Em 1828, chegou experimentalmente à lei que hoje leva seu nome (Lei de $\mathrm{Ohm}$ ): queda de tensão no circuito $\Delta \mathrm{V}=\mathrm{Ri}$. Um condutor com $\mathrm{R}=1 \mathrm{ohm}(\Omega)$, quando existe um $\Delta \mathrm{V}=1 \mathrm{~V}$, estabelece uma corrente $\mathrm{i}=1 \mathrm{~A}$. 
A taxa de aumento da energia magnética será: $\frac{d E_{B}}{d t}=L i \frac{d i}{d t}$, ver equação (2) anterior.

A energia magnética necessária para aumentar a corrente de zero até o valor i será: $E_{B}=\int_{0}^{E_{B}} d E_{B}=\int_{0}^{i} L i d i=\frac{1}{2} L i^{2}$, Equação (3), que será mais bem esclarecida na Seção 5.22, Equação (1a), é deduzido de maneira semelhante ao que foi deduzida a energia do campo elétrico $\mathrm{E}_{\mathrm{e}}$, ver Seção 5.19, Equação (1). Também se pode verificar a analogia com MHS.

\subsection{OSCILAÇÕES DO CIRCUITO INDUTÂNCIA-CAPACITÂNCIA LC}

Recordemos o Movimento Harmônico Simples (MHS) no exemplo do sistema massa-mola da Seção 2.1.3. Vimos que a força restauradora que faz a massa retornar quando estendida ou comprimida provem da lei de Hooke, $F=-k x$. O sinal negativo significa que a força tem direção contrária ao sentido crescente, em módulo, do deslocamento da massa. Lembremos que a origem está na massa em posição de equilíbrio, havendo uma direção positiva e outra negativa.

A seguir, algumas considerações energéticas do MHS.

Conforme a $2^{\text {a }}$ lei de Newton:

$$
F=-k x=m a=m v \frac{d v}{d x} \quad \therefore m v d v=-k x d x, \mathrm{a}=\frac{\mathrm{dv}}{\mathrm{dt}}=\frac{\mathrm{dv}}{\mathrm{dx}} \frac{\mathrm{dx}}{\mathrm{dt}}=\mathrm{v} \frac{\mathrm{dv}}{\mathrm{dx}}, \mathrm{v}=\frac{\mathrm{dx}}{\mathrm{dt}}
$$

Integrando entre a posição de equilíbrio, em que $x=0$ e a velocidade é $v_{0}$ e uma posição genérica $\mathrm{x}$ quando a velocidade genérica é $\mathrm{v}$, teremos: $\int_{v_{0}}^{v} m v d v=-\int_{0}^{x} k x d x$

$$
\frac{1}{2} m v^{2}-\frac{1}{2} m v_{0}^{2}=-\frac{1}{2} k x^{2} \quad \therefore \frac{1}{2} m v^{2}+\frac{1}{2} k x^{2}=\frac{1}{2} m v_{0}^{2}
$$

Conclui-se que, sendo $\frac{1}{2} \mathrm{mv}_{0}^{2}$ a energia cinética inicial, em que a energia potencial é nula, pois a mola ainda não foi acionada, esta representa a energia total do sistema, que é conservada. Essa energia será igual, em uma posição genérica, à soma da energia cinética da massa mais a energia potencial elástica da mola distendida ou contraída [ver figura da Equação (3a) na Seção 2.1.3].

A equação que rege o MHS é: $\frac{d^{2} x}{d t^{2}}+\frac{k}{m} x=0$ (1)

Cuja solução é: $\mathrm{x}=\mathrm{R} \cos \omega \mathrm{t}$, em que R é a amplitude máxima (valor máximo em módulo de $\pm x$ ) e o período (tempo de um ciclo) é:

$$
T=2 \pi \sqrt{\frac{m}{k}}(\text { ver em MHS, Seção 2.1.3, Equações } 4 \text { e 5). }
$$

Vamos verificar que existe uma analogia entre esse MHS e as oscilações $L C$. 


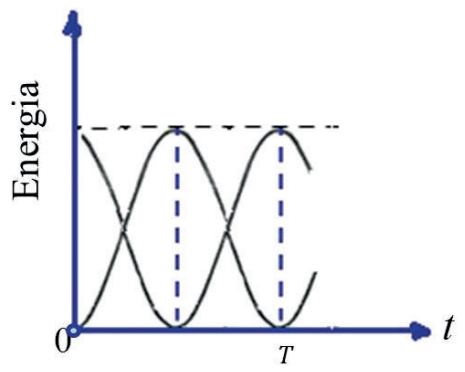

Sendo $U=$ Energia potencial do MHS $=\frac{1}{2} \mathrm{kx}^{2}$

$K=$ Energia cinética do MHS $=\frac{1}{2} \mathrm{mv}^{2}$

$R=$ Amplitude máxima

$E_{t}=K+U$

$\mathrm{K}+\mathrm{U}=\mathrm{K}_{\max }=\mathrm{U}_{\max }=\frac{1}{2} \mathrm{kR}^{2}=\mathrm{E}_{\mathrm{t}}=\frac{1}{2} \mathrm{~m} \mathrm{v}_{0}^{2}$

$\mathrm{U}=\mathrm{U}_{\max } \cos ^{2} \omega \mathrm{t}{ }^{44}$

$\mathrm{K}=\mathrm{K}_{\max } \operatorname{sen}^{2} \omega \mathrm{t}{ }^{45}$

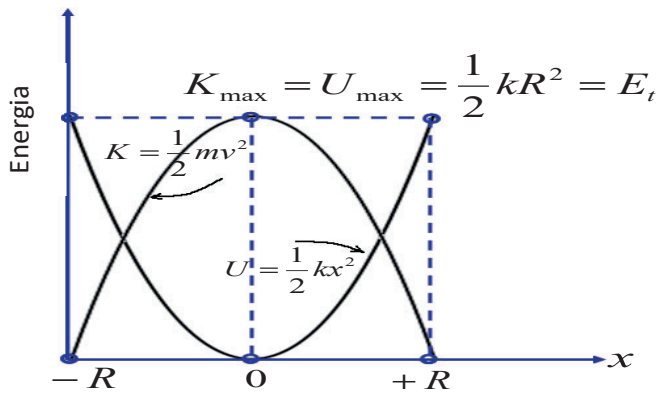

Energia de um oscilador MHS massa-mola.

${ }^{44} \mathrm{x}=\mathrm{R} \cos \omega \mathrm{t}$, ver seç̧ão 2.1.3, MHS equação $4 \mathrm{~b} 2, \therefore \mathrm{U}=\frac{1}{2} \mathrm{kR}^{2} \cos ^{2} \omega \mathrm{t}=\mathrm{U}_{\max } \cos ^{2} \omega \mathrm{t}$.

${ }^{45}$ Da mesma forma: $\mathrm{v}=\frac{\mathrm{dx}}{\mathrm{dt}}=-\omega \mathrm{R} \operatorname{sen} \omega \mathrm{t} \therefore \mathrm{K}=\frac{1}{2} \mathrm{~m} \omega^{2} \mathrm{R}^{2} \operatorname{sen}^{2} \omega \mathrm{t}=\frac{1}{2} \mathrm{kR}^{2} \operatorname{sen}^{2} \omega \mathrm{t}=\mathrm{K}_{\max } \operatorname{sen}^{2} \omega \mathrm{t}, \mathrm{m} \omega^{2}=\mathrm{k}$, Equação (4a) do MHS. 
Utilizando o princípio da conservação da energia para oscilações ${ }_{L C C}$ (ver Seções 5.19 e 5.21):

$$
\mathrm{E}_{\mathrm{t}}=\mathrm{E}_{\mathrm{e}}+\mathrm{E}_{\mathrm{B}}=\frac{1}{2} \mathrm{Li}^{2}+\frac{1}{2} \frac{\mathrm{q}^{2}}{\mathrm{C}}=\text { constante }(1 \mathrm{a})
$$

Derivando:

$$
\frac{d E_{t}}{d t}=\frac{d}{d t}\left(\frac{1}{2} L i^{2}+\frac{1}{2} \frac{q^{2}}{C}\right) \Rightarrow L i \frac{d i}{d t}+\frac{q}{C} \frac{d q}{d t}=0
$$

Porém, $i=\frac{d q}{d t} \quad$ e $\quad \frac{d i}{d t}=\frac{d^{2} q}{d t^{2}}$

Substituindo: $L \frac{d^{2} q}{d t^{2}}+\frac{1}{C} q=0$

Equação diferencial de $2^{\mathrm{a}}$ ordem.

Matematicamente idêntica a $m \frac{d^{2} x}{d t^{2}}+k x=0$ da massa-mola.

Solução: $\mathrm{x}=\mathrm{R} \cos \omega \mathrm{t}$

Portanto, para as oscilações $L$ : $q=q_{\max } \cos \omega t$

$$
\frac{\mathrm{dq}}{\mathrm{dt}}=\mathrm{i}=-\omega \mathrm{q}_{\max } \operatorname{sen} \omega \mathrm{t} \quad \frac{\mathrm{d}^{2} \mathrm{q}}{\mathrm{dt}^{2}}=-\omega^{2} \mathrm{q}_{\max } \cos \omega \mathrm{t}
$$

Substituindo: $-\mathrm{L} \omega^{2} \mathrm{q}_{\max } \cos \omega \mathrm{t}+\frac{1}{\mathrm{C}} \mathrm{q}_{\max } \cos \omega \mathrm{t}=0$

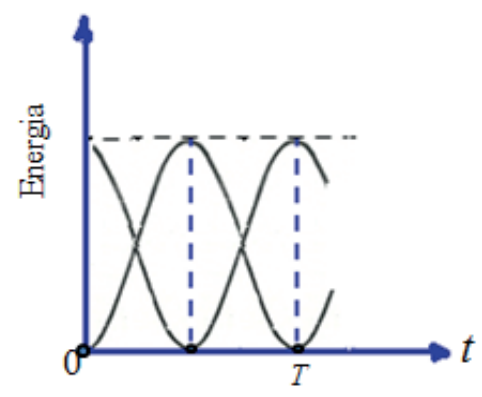

Simplificando: $\omega=\sqrt{\frac{1}{L C}}$ 
Como: $\omega=\frac{2 \pi}{T}$, ver Equação (3c), Capítulo 2, MHS.

Temos: $T=2 \pi \sqrt{L C}$ (período)

$\mathrm{E}_{\mathrm{B}}+\mathrm{E}_{\mathrm{e}}=\mathrm{E}_{\mathrm{B}_{\max }}=\mathrm{E}_{\mathrm{e}_{\max }}=\frac{\mathrm{q}^{2} \max }{2 \mathrm{C}}=\mathrm{E}_{\mathrm{t}}$

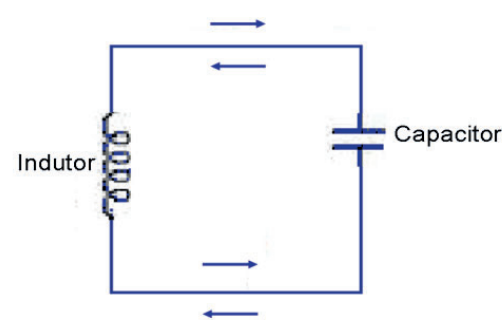

$$
\mathrm{E}_{\mathrm{e}}=\mathrm{E}_{\mathrm{e}_{\max }} \cos ^{2} \omega \mathrm{t}
$$

$$
\mathrm{E}_{\mathrm{B}}=\mathrm{E}_{\mathrm{B}_{\max }} \operatorname{sen}^{2} \omega \mathrm{t}
$$

O indutor armazena energia magnética, e o capacitor armazena energia elétrica. A partir disso, temos

\begin{tabular}{|c|c|c|c|}
\hline \multicolumn{2}{|c|}{ Mecânicas } & \multicolumn{2}{|c|}{ Eletromagnéticas } \\
\hline Mola & $U=\frac{1}{2} k x^{2}$ & Capacitor & $E_{e}=\frac{1}{2} \frac{q^{2}}{C}$ \\
\hline Massa & $K=\frac{1}{2} m v^{2}$ & Indutor & $E_{B}=\frac{1}{2} L i^{2}$ \\
\hline
\end{tabular}
a seguinte analogia com as fórmulas de energia:

Grandezas eletromagnéticas que correspondem a grandezas mecânicas:

\begin{tabular}{|l|l}
\hline$v=\frac{d x}{d t}$ & $i=\frac{d q}{d t}$ \\
\hline
\end{tabular}

$q$ corresponde ax

i corresponde a $\mathrm{v}$

$C$ corresponde a $1 / k$

${ }_{L}$ corresponde a m 
Naturalmente, o que acabamos de apresentar são casos ideais, nos quais não existe dissipação de energia por atrito ou resistência elétrica. No caso geral, devemos admitir que elas existam.

Seja o circuito $L C l$ em que $R$ simboliza as perdas pela resistência elétrica transformadas em energia térmica.

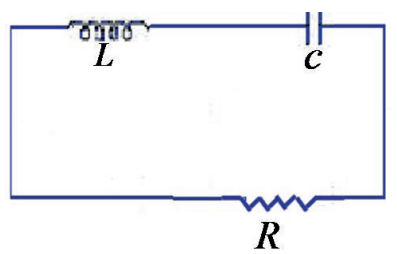

Então teremos, em lugar do que existe na Equação (1b), o seguinte:

$$
\frac{d E_{t}}{d t}=-i^{2} R \text { [ver Seção 5.21, Equação (2)] }
$$

$$
\frac{\mathrm{dE}_{\mathrm{t}}}{\mathrm{dt}}=\lim _{\mathrm{n} \rightarrow \infty} \frac{\Delta \mathrm{E}_{\mathrm{t}}}{\Delta \mathrm{t}}
$$

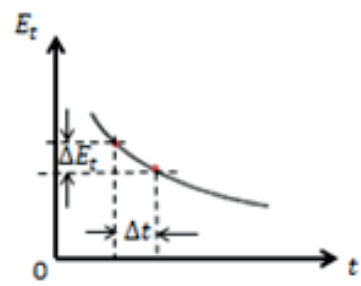

O sinal negativo significa que $E_{t}$ decresce com o tempo, e a perda é transformada em energia térmica por efeito Joule. ${ }^{46}$

A equação diferencial se torna: $L \frac{d^{2} q}{d t^{2}}+R \frac{d q}{d t}+\frac{1}{C} q=0$ pois: $\frac{\mathrm{dE}_{\mathrm{t}}}{\mathrm{dt}}=\mathrm{Li} \frac{\mathrm{di}}{\mathrm{dt}}+\frac{\mathrm{q}}{\mathrm{C}} \mathrm{i}=-\mathrm{Ri}^{2}$

As oscilações tornam-se amortecidas, tendendo a se anular.

Para evitar isso, a energia dissipada deve ser reposta, intercalando no circuito uma fem $\Delta V_{e}$, que varia senoidalmente. Nesse caso, teremos oscilações forçadas.

Pela lei de Ohm:

$$
-R i=\Delta V_{L}+\Delta V_{C}-\Delta V_{e} \Rightarrow \Delta \mathrm{V}_{\mathrm{L}}+\Delta \mathrm{V}_{\mathrm{C}}+\mathrm{Ri}=\Delta \mathrm{V}_{\mathrm{E}}
$$

\footnotetext{
${ }^{46}$ Enquanto $\Delta \mathrm{t}$ cresce, $\Delta \mathrm{E}_{\mathrm{t}}$ decresce, logo, como: $\mathrm{dt}$ e $\mathrm{dE}_{\mathrm{t}}$, têm sinais contrários $\therefore$ o sinal negativo.
} 


$$
\Delta V_{e}=\Delta V_{e_{o}} \operatorname{sen} \omega_{f} t_{47}
$$

$\omega_{\mathrm{f}}$ é a velocidade angular da fem $\Delta \mathrm{V}_{\mathrm{e}}$, oscilante.

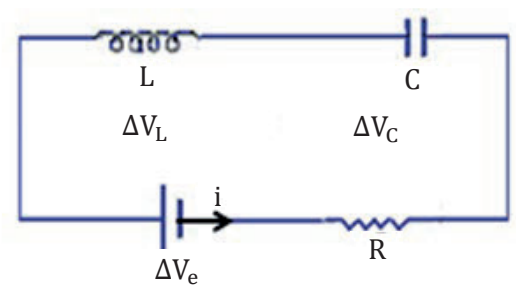

A potência média será máxima quando $\omega_{f}=\omega$ onde $\omega$ frequência característica do circuito $L C$ e dizemos que o circuito está em "ressonância”. (1e)

$\phi=$ diferença de fase entre corrente i e $\Delta \mathrm{V}_{\mathrm{e}}$, que é a diferença de potencial, fem aplicada.

$$
\mathrm{i}=\mathrm{i}_{\max } \operatorname{sen}\left(\omega_{\mathrm{f}} \mathrm{t}-\phi\right)
$$

Reatância do circuito: $X=X_{L}-X_{c}(1 f)$, em que $X_{L}=\omega_{f} L$, é reatância indutiva e $X_{c}=1 / \omega_{f} C$, reatância capacitiva.

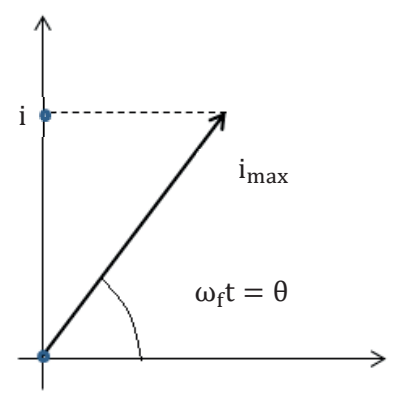

Impedância: $\mathrm{Z}=\sqrt{\mathrm{R}^{2}+\mathrm{X}^{2}}$.

Graficamente: 


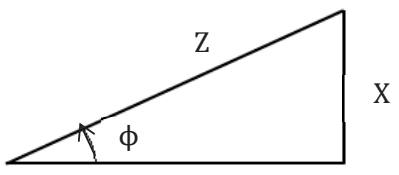

$\mathrm{R}$

Em que:

$$
\phi=\arctan \frac{x}{R}
$$

$R=$ resistência

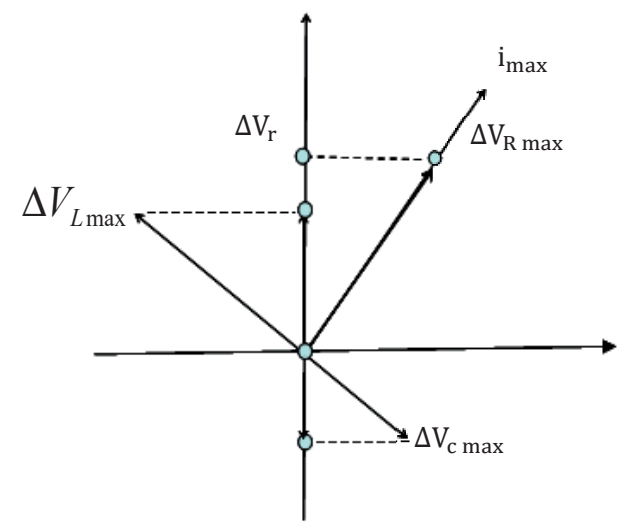

$$
\Delta \mathrm{V}_{\mathrm{Rmax}}=i_{R \max } R
$$

Em fase $\phi=0 \quad$ sen $\omega_{\mathrm{f}} \mathrm{t}$

$\mathrm{C}=$ capacidade

$i_{c}$ avançado $90^{\circ}$ sobre $\Delta V_{c}$

$X_{C}=$ reatância capacitiva

$\phi=-90^{\circ}$

$\operatorname{sen}\left(\omega_{\mathrm{f}} t+90^{\circ}\right)$

$\mathrm{X}_{\mathrm{c}}=\frac{1}{\omega_{\mathrm{f}} \mathrm{C}} \quad \Delta \mathrm{V}_{\mathrm{C}_{\max }}=\mathrm{i}_{\mathrm{C}_{\max }} \mathrm{X}_{\mathrm{C}}$ 


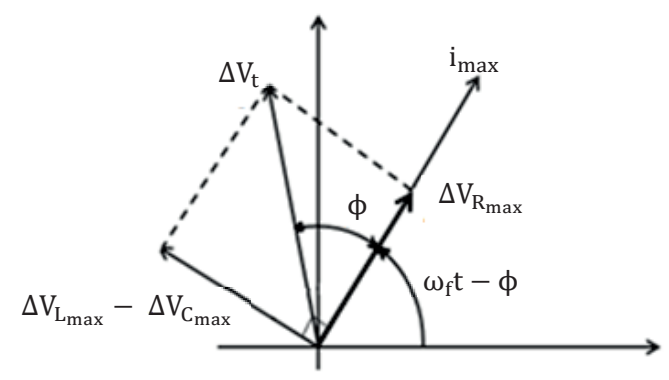

$L=$ indutância $\quad \mathrm{i}_{\mathrm{L}}$ atrasado $90^{\circ}$ sobre $\Delta V_{L}$

$X_{L}=$ reatância indutiva

$\phi=+90^{\circ}$

$\operatorname{sen}\left(\omega_{\mathrm{f}} \mathrm{t}-90^{\circ}\right)$

$X_{L}=\omega_{f} L$

$\Delta V_{L_{\max }}=i_{L_{\max }} X_{L}$

$\Delta \mathrm{V}_{\mathrm{e}}^{2}=\Delta \mathrm{V}_{\mathrm{r}_{\max }^{2}}^{2}+\left(\Delta \mathrm{V}_{\mathrm{L}_{\max }}-\Delta \mathrm{V}_{\mathrm{c}_{\max }}\right)^{2}$

$Z$ = impedância

$Z=\sqrt{R^{2}+\left(X_{L}-X_{c}\right)^{2}}$, ver equação $1 \mathrm{f}, \mathrm{X}=\mathrm{X}_{\mathrm{L}}-\mathrm{X}_{\mathrm{C}}$, reatância.

$$
i_{\max }=\frac{\Delta V_{t}}{Z} \quad \tan \phi=\frac{\Delta V_{L \max }-\Delta V_{c \max }}{\Delta V_{R \max }}=\frac{X_{L}-X_{c}}{R} \tan \phi=\frac{\Delta V_{L}-\Delta V_{c}}{\Delta V_{R}}
$$

A Potência média (também chamada Potência eficaz) é:

$$
P_{m e d}=\frac{1}{2} I^{2} R=\left(\frac{i_{\max }}{\sqrt{2}}\right)^{2} R \quad \therefore P_{m e d}=\Delta V_{r m s} i_{r m s} \cos \phi
$$

Sendo: $\mathrm{i}_{\mathrm{rms}}=\frac{\mathrm{i}_{\max }}{\sqrt{2}}{ }^{48} \quad \Delta \mathrm{V}_{\mathrm{rms}}=\Delta \mathrm{V}_{\mathrm{R}_{\max }} / \sqrt{2} \quad \cos \phi=\frac{\Delta \mathrm{V}_{\mathrm{R}_{\max }}}{\Delta \mathrm{V}_{\mathrm{e}}}=\frac{\mathrm{R}}{\mathrm{Z}}$, ver Equação (3)

$\cos \phi=$ fator de potência

${ }^{48}$ i não varia linearmente de 0 a $\mathrm{i}_{\max }$, mas de $0 \mathrm{a} \mathrm{i}^{2}$ max , por isso a utilização da média quadrática: $\mathrm{i}_{\text {med }}=\sqrt{\frac{0+\mathrm{i}^{2} \text { max }}{2}}$. Ver média quadrática em Estatística. 
rms = root mean square $=$ valor médio quadrático

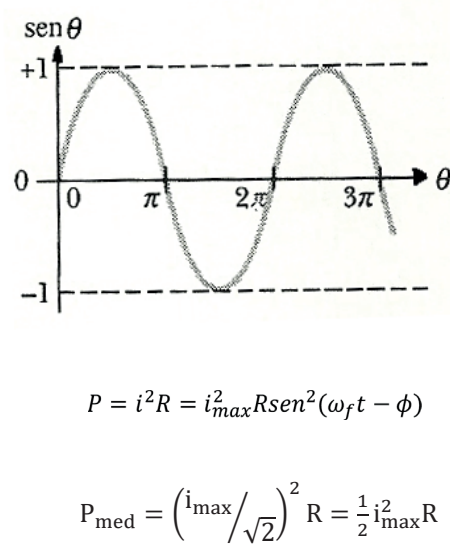

O valor médio em um ciclo é nulo.

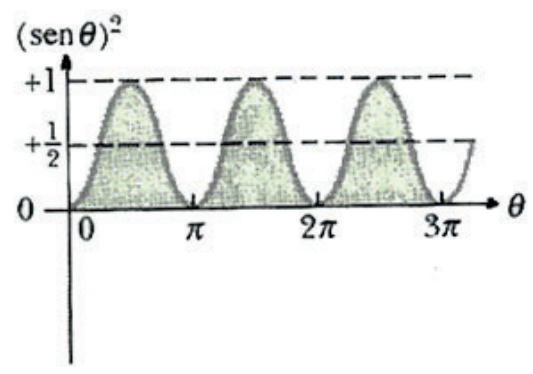

Valor médio em um ciclo: $1 / 2$

Na ressonância: $X_{L}-X_{c}=\omega L-1 / \omega C=0, \quad$ pois $i_{\max }=\frac{\Delta V_{e}}{R}$ e também $Z=R$. Recordar que na ressonância $\omega_{\mathrm{f}}=\omega$.

Logo: $\frac{1}{\omega C}=\omega L \therefore \omega=\frac{1}{\sqrt{L C}}$ o mesmo valor achado para o circuito $L C$, ver Equação $(1 \mathrm{c})$.

Para simplificar, denotamos a potência média $\mathbf{P}_{\mathbf{m}} \operatorname{com} \mathbf{P}$ e $\mathbf{i}_{\mathbf{r m s}}$ e $\Delta \mathbf{V}_{\mathbf{r m s}} \operatorname{com} \mathbf{I}$ e $\mathbf{V}$. A potência média se torna: $\mathbf{P}=\mathbf{P}_{\mathrm{m}}=\frac{\mathbf{i}_{\max } \Delta \mathbf{V}_{\mathbf{R}_{\max }}}{2} \cos \phi$.

$\therefore \mathrm{P}=\mathrm{VI} \cos \phi$. 
Para medir o fator de potência $\cos \phi$, utilizamos simultaneamente um voltímetro, um amperímetro e um wattímetro.

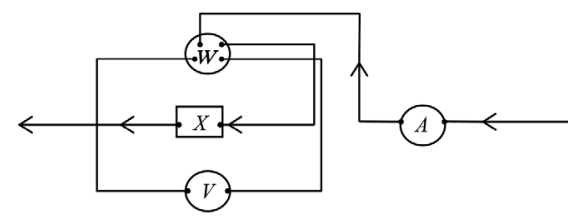

\subsubsection{Exemplos ilustrativos}

Nas redes domésticas e industriais, temos exemplos do que foi apresentado. A rede em si, considerando as fiações, é resistiva, assim como lâmpadas e chuveiros, fornos e aquecedores elétricos; os motores a indução usados em refrigeradores, liquidificadores, ar-condicionado, máquina de lavar roupa, pratos, têm carga indutiva. A entrada da rede doméstica é com diferença de potencial, geralmente chamada de tensão elétrica, de $110 \mathrm{~V}$ para iluminação e $220 \mathrm{~V}$ para força. A fem aplicada que vem da rua através de transformadores abaixadores de tensão, ${ }^{49}$ distribui a energia elétrica que costuma vir das hidrelétricas.

Nas redes elétricas industriais, utilizam-se tensões geralmente de $220 \mathrm{~V}, 380 \mathrm{~V}$ e até $3.800 \mathrm{~V}$, conforme seu porte. Além disso, as redes industriais normalmente são trifásicas.

Essas redes são de corrente alternada, como, aliás, foi sugerido no desenvolvimento teórico. Em todo circuito oscilante, a corrente elétrica que o percorre é alternada. Os circuitos normalmente não são ressonantes.

Por outro lado, o circuito oscilante é amplamente utilizado em telecomunicações e, normalmente, em ressonância, conforme visto no texto indicado com (1e), entre aspas. ${ }^{50}$

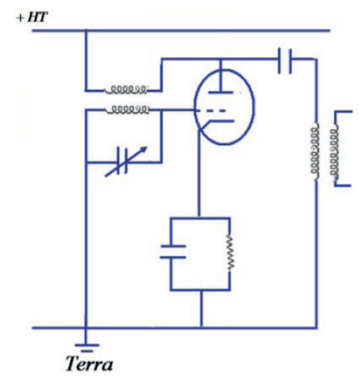

Como exemplo, temos um oscilador em telecomunicações usando válvula triodo.

\footnotetext{
49 Inclusive linhas de transmissão, incluindo as linhas de alta tensão. Em todos esses, naturalmente, ocorrem perdas.

50 A ressonância é encontrada sempre em um sistema, por exemplo, quando sintonizamos o rádio em uma estação transmissora. As emissoras transmitem com oscilações forçadas, porém, cada ajuste do sintonizador corresponde uma frequência natural do receptor. Quando essa frequência coincide com a da estação transmissora, a energia absorvida é máxima e essa é a única estação que ouvimos.
} 
Esquematicamente:

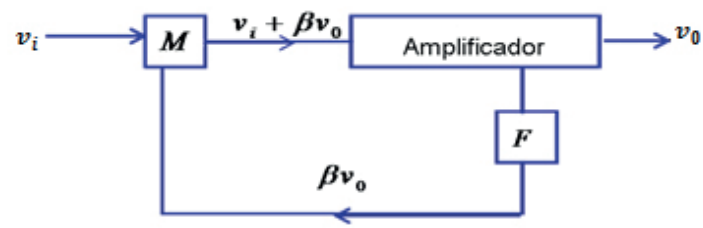

Há uma "realimentação positiva”. Uma fração $\beta v_{0}$ do sinal de saída $(\beta<1)$ é selecionada pelo circuito de realimentação $(\mathrm{em} F$ ). Esse sinal retorna para a entrada, onde é misturado (em $M)$ com o sinal original de entrada (em $M$ ) para reforçá-lo, compensando as perdas resistivas.

Ganho do amplificador é: $g=\frac{v_{0}}{v_{i}+\beta v_{0}}$

Há ganho de voltagem e amperagem, pois a impedância do circuito é constante.

Ganho efetivo: $g^{\prime}=\frac{v_{0}}{v_{i}}=\frac{\mathrm{g}}{v_{\mathrm{i}}}\left(v_{\mathrm{i}}+\beta v_{0}\right)=\mathrm{g}\left(1+\beta \frac{v_{0}}{v_{\mathrm{i}}}\right)=\mathrm{g}\left(1+\beta \mathrm{g}^{\prime}\right)$

Combinando os dois: $\mathrm{g}^{\prime}=\mathrm{g}+\beta \mathrm{g} \mathrm{g}^{\prime} \Rightarrow \mathrm{g}^{\prime}(1-\beta \mathrm{g})=\mathrm{g}$

$$
g^{\prime}=\frac{g}{1-\beta g}
$$

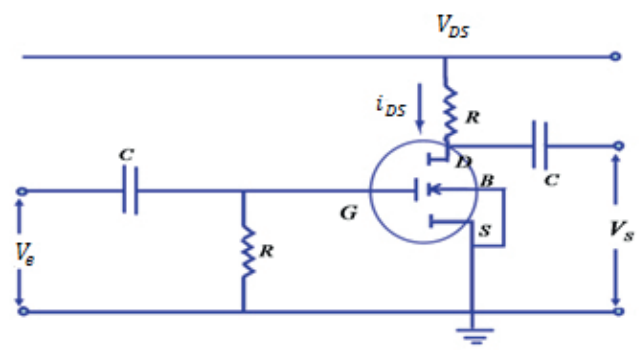

A válvula triodo é amplificadora. Atualmente, usamos um transistor de efeito de campo semicondutor metal-óxido (MOSFET) como amplificador.

Apresentamos as propriedades piezelétricas e a manutenção de uma frequência constante do quartzo, na Seção 2.1.6, “Quartzo”. 


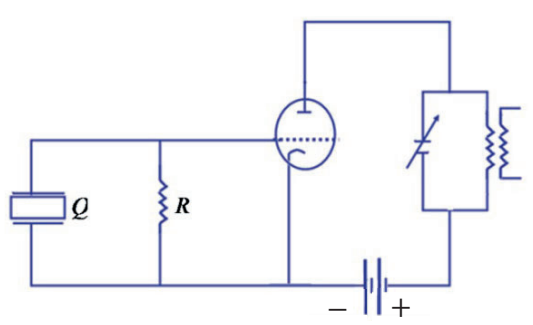

Oscilador de emissora

A placa de quartzo $Q$, cortada normalmente ao eixo principal do cristal, é colocada entre as placas de um condensador. A lâmina de quartzo tem uma frequência de oscilação própria, que é função da espessura $\delta$ do cristal. O comprimento de onda é $\lambda=103,6 \delta$ ( $\lambda$ em $m$ e $\delta$ em $\mathrm{mm}$ ). A capacidade do condensador oscilará com o mesmo ritmo, sintonizando-se o circuito anódico com a frequência. As oscilações do quartzo se sustentam devido ao retroacoplamento da capacidade grade-anodo (placa); HÜTTE.

\subsection{OSCILAÇÕES ELETROMAGNÉTICAS}

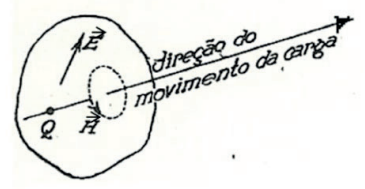

Uma carga elétrica $Q$ produz ao seu redor um campo elétrico $\vec{E}$. Se a carga elétrica estiver em movimento, ocorrerá uma corrente elétrica.

Como a corrente elétrica produz ao seu redor um campo magnético $\vec{H}$, concluímos que uma carga elétrica em movimento produz ao seu redor dois campos: o elétrico, que existe sempre, e o magnético, produzido por estar em movimento.

Segundo a teoria quântica, a corrente elétrica, que em um condutor é formada essencialmente de elétrons livres em movimento, faz elétrons livres colidirem com os elétrons que orbitam nos átomos. Com o choque, o elétron da órbita escapa e a órbita fica vaga de um elétron. O elétron de uma órbita mais afastada salta para a órbita vaga. Ao fazê-lo, perde energia, que escapa do átomo na forma de um quantum de energia, que Einstein chamou de fóton na sua teoria do efeito fotoelétrico, confirmando o que Planck afirmara a respeito de a energia ser composta de "pacotes de energia" denominados quanta (plural de quantum em latim). O quantum é uma quantidade de energia indivisível, isto é, a menor parcela possível de energia. Os fótons oscilam transversalmente à propagação das ondas que são formadas. A velocidade de propagação é a da luz, cerca de $300.000 \mathrm{~km} / \mathrm{s}$ no vácuo. Seu valor mais aceito é de 
$299.792,458 \mathrm{~km} / \mathrm{s}$. Mais detalhes da teoria dos quanta serão apresentados oportunamente (ver Anexo 6, equação 0 e páginas seguintes).

Conclui-se, então, que existe uma relação entre a corrente alternada e a frequência das ondas eletromagnéticas resultantes, entre outras características.

Em 1832, Kohlrausch e Weber determinaram a relação das unidades eletrostáticas e eletromagnéticas chegando a um valor próximo da velocidade da luz. Levando em consideração essas experiências, Maxwell em sua obra Tratado de Eletricidade e Magnetismo, de $1873^{51}$ [no final desta Seção 5.23, Equação (5)], deduziu essa relação de forma matemática, mostrando como ela surgia, ver também na Secção 5.23.1, Velocidade das ondas eletromagnéticas no vácuo.

Nas palavras de Maxwell, a velocidade da ondulação transversal em nosso meio hipotético, calculada das experiências eletromagnéticas de Kohlrausch e Weber, concorda tão exatamente com a velocidade da luz das experiências óticas de M. Fizeau, que mal podemos evitar a conclusão de que a "luz consiste em ondulações transversais do mesmo meio, que é a fonte dos fenômenos elétricos e magnéticos”. Essas palavras refletem o raciocínio e o vocabulário daquela época.

Considerando um ponto $P$, bastante afastado da fonte emissora, de modo que as frentes de onda passando sejam essencialmente planas. Os campos elétrico e magnético são perpendiculares entre si e ambos perpendiculares à direção de propagação. Façamos o eixo $x$, direção da propagação e $y$ e $z$ paralelos aos campos $\vec{E}$ e $\vec{B}$.(a)

Esses campos se desenvolvem da seguinte forma: quando $\vec{E}$ é máximo, $\vec{B}$ é nulo e vice-versa. Isso está de acordo com a distribuição das energias em um circuito $L C$ (ver Seção 5.22), pois as intensidades dos campos elétrico e magnético são proporcionais às respectivas energias. ${ }^{52}$

$$
\text { Portanto: } \begin{array}{rlrl}
E_{x} & =0 & \mathrm{E}_{\mathrm{y}}=\mathrm{E} & E_{z}=0 \\
B_{x}=0 & \mathrm{~B}_{\mathrm{y}}=0 & B_{z}=B
\end{array}
$$

Suponhamos os campos no vácuo. Logo, não há cargas livres nem correntes: $\rho=0$ e $\rho v=0$, pois $\rho v=d i / d S$, levando em conta que: $\rho=\mathrm{dq} / \mathrm{dV}$ e i $=-\mathrm{dq} / \mathrm{dt}$ (ver Seções 5.9 e 5.16) e diferenciando:

$$
d \oint_{S} \rho \vec{v} \cdot \vec{n} d S=d i \Rightarrow \frac{d i}{d S}=\rho \vec{v} \cdot \vec{n}
$$

$\overrightarrow{\mathrm{n}}$ vetor unitário normal à superfície dS (ver Seção 5.4).

${ }^{51}$ Mencionada na Seção 5.16, na página inicial da Secção 5.16.

52 Quando a energia elétrica está preenchida, a energia magnética está vazia e vice-versa. Há troca de energia entre campo elétrico e campo magnético. A energia total é conservada. Veremos na Seção 5.26, Equações 5.7 e 5.8 que as energias eletromagnéticas são proporcionais, respectivamente, aos quadrados de $\vec{E}$ e $\vec{B}$. 


\subsubsection{Lei de Gauss para o campo elétrico}

$$
\frac{\partial E_{x}}{\partial x}+\frac{\partial E_{y}}{\partial y}+\frac{\partial E_{z}}{\partial z}=\frac{\rho}{\varepsilon_{0}}=0 \quad \therefore \frac{\partial E}{\partial y}=0, \text { ver final da Secção } 5.10 \text { e anterior. }
$$

\subsubsection{Lei de Gauss para o campo magnético}

$$
\frac{\partial B_{x}}{\partial x}+\frac{\partial B_{y}}{\partial y}+\frac{\partial B_{z}}{\partial z}=0 \quad \therefore \frac{\partial B}{\partial z}=0, \text { ver final da Secção } 5.12 \text { e anterior. }
$$

\subsubsection{Lei de Faraday-Henry}

$$
\begin{array}{ll}
\frac{\partial E_{x}}{\partial z}-\frac{\partial E_{z}}{\partial x}=-\frac{\partial B_{y}}{\partial t}=0 & \therefore \frac{\partial E}{\partial z}=0, \text { ver final da Seção } 5.15 .2 \text { e anterior } \\
\frac{\partial E_{y}}{\partial x}-\frac{\partial E_{x}}{\partial y}=-\frac{\partial B_{z}}{\partial t} & \therefore \frac{\partial E}{\partial x}=-\frac{\partial B}{\partial t}, \text { ver final da Seção } 5.15 .2 \text { e anterior }(0)
\end{array}
$$

\subsubsection{Lei de Ampère-Maxwell}

$$
\begin{array}{cc}
\frac{\partial B_{z}}{\partial y}-\frac{\partial B_{y}}{\partial z}=\varepsilon_{0} \mu_{0} \frac{\partial E_{x}}{\partial t}=0 & \therefore \frac{\partial B}{\partial y}=0, \text { ver final da Seção } 5.16 \text { e anterior } \\
\frac{\partial B_{x}}{\partial z}-\frac{\partial B_{z}}{\partial x}=\varepsilon_{0} \mu_{0} \frac{\partial E_{y}}{\partial t} & \therefore-\frac{\partial B}{\partial x}=\varepsilon_{0} \mu_{0} \frac{\partial E}{\partial t} \text {, ver final da Seção } 5.16 \text { e anterior (1) }
\end{array}
$$

$\vec{E}$ e $\vec{B}$ não dependem de $y$ ou $z$, pois variam com x e t, sendo constantes com y e z.

As intensidades dos campos $\vec{E}$ e $\vec{B}$ dependem de $x$ e t.

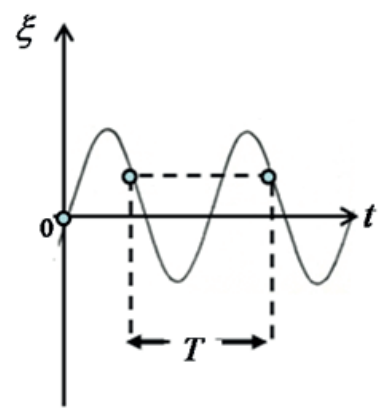

$$
T=\text { periodo }
$$

Têm o mesmo valor em todos os pontos pertencentes a planos perpendiculares ao eixo $x$. 
Seja a função senoidal: $\xi=\xi(x, t)=\xi_{0} s e k(x-v t)$

Vamos mostrar que satisfaz à equação diferencial:

$$
\frac{\partial^{2} \xi}{\partial t^{2}}=v^{2} \frac{\partial^{2} \xi}{\partial x^{2}}
$$

Em que: $\mathcal{V}=$ velocidade de propagação.

Temos novamente uma equação diferencial de $2^{\mathrm{a}}$ ordem como visto para o circuito $L C$ e o MHS [ver Seção 5.22, Equações (1b) e (2)].

$\xi=\xi(x, t)$ é uma função periódica, como já visto no MHS [Seção 2.1.3, Equação (4b1)], isto é, o valor de $\xi$ se repete após um intervalo de tempo T, chamado período.

Sendo $\xi=f(x-v t)$. Chamando $u=x-v t$

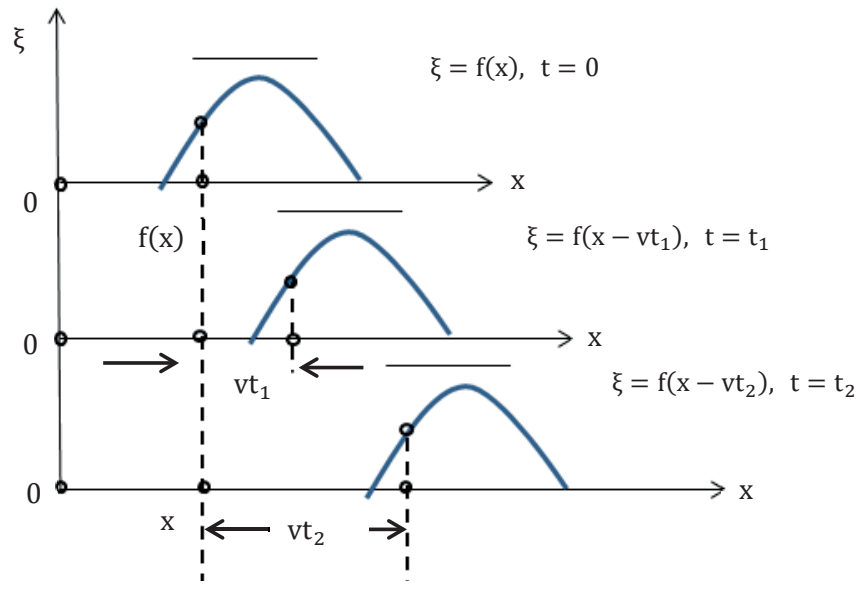

Teremos: $\frac{\partial u}{\partial x}=1 \quad \frac{\partial u}{\partial t}=-v$

$$
\frac{\partial \xi}{\partial x}=\frac{d \xi}{d u} \frac{\partial u}{\partial x}=\frac{d \xi}{d u}
$$

Pela regra de cadeia:

$$
\begin{gathered}
\therefore \frac{\partial \xi}{\partial t}=\frac{d \xi}{d u} \frac{\partial u}{\partial t}=-v \frac{d \xi}{d u} \\
\frac{\partial^{2} \xi}{\partial x^{2}}=\frac{d}{d u}\left(\frac{\partial \xi}{\partial x}\right) \frac{\partial u}{\partial x}=\frac{d^{2} \xi}{d u^{2}}
\end{gathered}
$$


Pois: $\frac{\partial \xi}{\partial \mathrm{x}}=\frac{\partial \xi}{\partial \mathrm{u}}$

$\frac{\partial^{2} \xi}{\partial t^{2}}=\frac{d}{d u}\left(\frac{\partial \xi}{\partial t}\right) \frac{\partial u}{\partial t}=-v \frac{d^{2} \xi}{d u^{2}}(-v)=v^{2} \frac{d^{2} \xi}{d u^{2}}$

Pois: $\frac{\partial \xi}{\partial t}=-v \frac{d \xi}{d u}$

Logo: $\frac{\partial^{2} \xi}{\partial \mathrm{t}^{2}}=\mathrm{v}^{2} \frac{\partial^{2} \xi}{\partial \mathrm{x}^{2}}$, já que $\frac{\partial \xi}{\partial \mathrm{x}}=\frac{\partial \xi}{\partial \mathrm{u}}$

E: $\frac{\partial^{2} \xi}{\partial \mathrm{x}^{2}}=\frac{\partial^{2} \xi}{\partial \mathrm{u}^{2}}$

Voltando às equações anteriores de $\vec{E}$ e $\vec{B}$ :

$$
\frac{\partial^{2} E}{\partial x^{2}}=-\frac{\partial^{2} B}{\partial x \partial t} \quad \text { e } \quad-\frac{\partial^{2} B}{\partial x \partial t}=\varepsilon_{0} \mu_{0} \frac{\partial^{2} E}{\partial t^{2}}
$$

Pois $\frac{\partial E}{\partial x}=-\frac{\partial B}{\partial t} \quad$ e $-\frac{\partial B}{\partial x}=\varepsilon_{0} \mu_{0} \frac{\partial E}{\partial t}$

Logo: $\frac{\partial^{2} E}{\partial t^{2}}=\frac{1}{\varepsilon_{0} \mu_{0}} \frac{\partial^{2} E}{\partial x^{2}}$

Comparando com a Equação (2) senoidal apresentada acima: $c=\frac{1}{\sqrt{\varepsilon_{0} \mu_{0}}}(5)$

Em que $c$, como será visto, é a velocidade da luz no vácuo.

Portanto: $E=E(x-c t)$

Também teremos: $\frac{\partial^{2} B}{\partial t^{2}}=\frac{1}{\varepsilon_{0} \mu_{0}} \frac{\partial^{2} B}{\partial x^{2}}(6) \quad B=B(x-c t)$

Consideraremos ondas harmônicas com frequência: $\quad \gamma=\omega / 2 \pi$ e comprimento de onda $\lambda=2 \pi / k$, onde $\mathrm{k}$ é o $\mathrm{n}^{\circ}$ de comprimentos de onda em $2 \pi$ rad [ver anexo A.6, páginas iniciais Equação da onda de Schrödinger, Equações (a) e (b)].

Obtemos: $\quad E=E_{0} \operatorname{sen} k(x-c t)=E_{0} \operatorname{sen}(\mathrm{kx}-\omega \mathrm{t})$

Veremos no Anexo 6 em Resolução da Equação da Onda, como resolver, Equações (8a) e (8b).

$$
B=B_{0} \operatorname{sen} k(x-c t)=B_{0} \operatorname{sen}(\mathrm{kx}-\omega \mathrm{t})
$$

Usamos $\omega=k c$, ver Anexo 6.4.1 “Em outras palavras”, Equação (0): 


$$
\therefore \frac{\partial E}{\partial x}=k E_{0} \cos k(x-c t) \quad \text { e } \frac{\partial B}{\partial t}=-k c B_{0} \cos k(x-c t)
$$

Como $\frac{\partial \mathrm{E}}{\partial \mathrm{x}}=-\frac{\partial \mathrm{B}}{\partial \mathrm{t}}$, ver Equação (0), lei de Faraday-Henry, teremos:

$$
\mathrm{E}_{0}=\mathrm{cB}_{0} \therefore \mathrm{E}=\mathrm{cB}
$$

\subsubsection{Velocidade das ondas eletromagnéticas no vácuo}

Sendo: $\mu_{0}=4 \pi \times 10^{-7}$ weber $/ \mathrm{amp} \cdot \mathrm{m}$ e $\varepsilon_{0}=8,9 \times 10^{-12} \mathrm{C}^{2} / \mathrm{Nm}^{2}$

(Ver Seção 5.1, desenvolvimento da fórmula de Coulomb para $\varepsilon_{0}$, e Seção 5.16 para $\mu_{0}$.)

$$
\begin{gathered}
1 \text { weber }=\text { Tesla. } m^{2}=m^{2} \mathrm{~kg} /(\mathrm{Cs}) \\
\text { Tesl, unidade de } B \quad 1 T=\mathrm{kg} /(\mathrm{Cs})
\end{gathered}
$$

$\mu_{0}=$ constante de permeabilidade no vácuo (ver Seção 5.24), como: $\mathrm{c}=\frac{1}{\sqrt{\varepsilon_{0} \mu_{0}}}$

Teremos: $\quad c=\frac{1}{\sqrt{\left(4 \pi \times 10^{-7}\right)\left(8,9 \times 10^{-12}\right)}}=3 \times 10^{8} \mathrm{~m} / \mathrm{s}$, velocidade das ondas eletromagnéticas no vácuo. (7)

Concordando, assim, com as medições de M. Fizeau para velocidade da luz no vácuo.

Análise dimensional: $\left[\mathrm{m}^{2} \cdot \mathrm{kg} \cdot \mathrm{s} /(\mathrm{C} \cdot \mathrm{s} \times \mathrm{C} \cdot \mathrm{m})\right] \times\left[\mathrm{C}^{2} / \mathrm{Nm}^{2}\right]=\left[\mu_{0}\right] \times\left[\varepsilon_{0}\right]=\frac{1}{\left[\mathrm{c}^{2}\right]}$

$$
=(\mathrm{kg} \cdot \mathrm{s} / \mathrm{m} \cdot \mathrm{s})(1 / \mathrm{N})=(\mathrm{kg} \cdot \mathrm{s} / \mathrm{m} \cdot \mathrm{s})\left(\mathrm{s}^{2} / \mathrm{kg} \cdot \mathrm{m}\right)=\mathrm{s}^{2} / \mathrm{m}^{2} \quad N=\mathrm{kg} \cdot \mathrm{m} / \mathrm{s}^{2}
$$

53 N é Newton, unidade de força no sistema MKSC, ver Seção 4.4. 


\subsection{FORÇAS ENTRE CORRENTES}

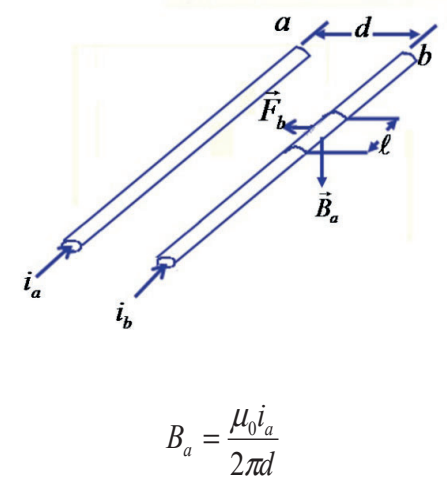

Pela lei de Ampère- Maxwell (ver Seção 5.16).

$$
\vec{F}_{b}=i_{b} \vec{\ell} \times \vec{B}_{a}
$$

Outra forma da lei da indução magnética, pois $d \vec{F}=d q \frac{d \vec{\ell}}{d t} \times \vec{B}=i d \vec{\ell} \times \vec{B}$ aplicando:

$\overrightarrow{\mathrm{F}}=\mathrm{q} \overrightarrow{\mathrm{v}} \times \overrightarrow{\mathrm{B}}, \quad$ ver Seção 5.11, sobre indução magnética e integrando: $\vec{F}=\overrightarrow{i \ell} \times \vec{B}$

Então, em módulo: $\mathrm{F}_{\mathrm{b}}=\mathrm{i}_{\mathrm{b}} \mathrm{l} \mathrm{B}_{\mathrm{a}}=\frac{\mu_{0} \mathrm{i}_{\mathrm{a}} \mathrm{i}_{\mathrm{b}}}{2 \pi \mathrm{d}}$, pois $\overrightarrow{\mathrm{l}} \times \overrightarrow{\mathrm{B}}_{\mathrm{a}}=1 \mathrm{~B}_{\mathrm{a}}$ sen $90^{\circ}$ e sen $90^{\circ}=1$.

Logo, podemos definir o Ampère: corrente que, circulando em dois condutores paralelos separados por uma distância de um metro, produz uma força, ${ }^{54}$ sobre cada condutor, de $2 \times 10^{-7} \mathrm{~N}$ por metro de comprimento de cada um deles:

$$
\frac{F}{\ell}=\frac{\mu_{0} i^{2}}{2 \pi d}=\frac{\left(4 \pi \times 10^{-7} \text { weber } / \mathrm{A} \cdot \mathrm{m}\right)(1 \mathrm{~A})^{2}}{2 \pi(1 \mathrm{~m})}=2 \times 10^{-7} \mathrm{~N} / \mathrm{m}
$$

Análise dimensional: $\frac{\left(\mathrm{m}^{2} \mathrm{~kg} \cdot \mathrm{s} / \mathrm{C}^{2} \mathrm{~m} \cdot \mathrm{s}\right)\left(\mathrm{C}^{2} / \mathrm{s}^{2}\right)}{\mathrm{m}}=\mathrm{kg} \cdot \mathrm{m} / \mathrm{s}^{2} \mathrm{~m}=\mathrm{N} / \mathrm{m}$, ver $\mathrm{N}$ no final da Seção 5.23.5.

$\mu_{0}=$ constante de permeabilidade no vácuo com valor atribuído de: $\mu_{0}=4 \pi \times 10^{-7}$ weber $/$ A.m para fornecer a força $F / \ell$ desejada (ver Secção 5.23.5).

Na prática, utiliza-se uma balança de corrente. Um dos pratos é conectado a duas bobinas que se inter-relacionam, em vez de dois fios, por onde passa a mesma corrente. Surge então, uma força que age

\footnotetext{
${ }^{54}$ Se as correntes tiverem o mesmo sentido, teremos em cada um, força de atração.
} 
no prato e que deve ser contrabalançada no outro prato com pesos. Estes fornecem a força, que deve ser calculada com outra fórmula, por se tratar de espiras.

Se tomarmos a fórmula $\mathrm{F}=\mu_{0} \ell \mathrm{i}^{2} / 2 \pi \mathrm{d}$ e fizermos $K_{m}=\frac{\mu_{0}}{4 \pi}$, obteremos: $\mathrm{F}=\mathrm{K}_{\mathrm{m}} \frac{2 \mathrm{i}^{2}}{\mathrm{~d}} \ell$.

Como vimos na Secção 5.1: $K_{e}=1 / 4 \pi \varepsilon_{0}$, recorde-se que $\mu_{0}=4 \pi \times 10^{-7}$ weber/A.m (ver Seção 5.23.5, sobre a velocidade das ondas eletromagnéticas no vácuo).

Relacionando os dois: $\frac{K_{e}}{K_{m}}=\frac{1}{\varepsilon_{0} \mu_{0}}=c^{2}$ como visto na Secção 5.23.5.

$\mathrm{K}_{\mathrm{e}}=\mathrm{K}_{\mathrm{m}} \mathrm{c}^{2}=10^{-7} \mathrm{c}^{2}$. Isso explica a escolha para $K_{e}$ na Secção 5.1.

Então, c é a velocidade da onda eletromagnética e igual à velocidade da luz. Para Maxwell, esse foi o primeiro indício de que a luz é uma onda eletromagnética. Heinrich Hertz provou que as ondas eletromagnéticas têm a velocidade da luz. A experiência foi feita com oscilador por meio de transformador com bobina de Ruhmkorff (SALMERON, ALONSO, FINN). A previsão de Maxwell conduziu ao conceito de espectro eletromagnético e à descoberta das ondas de radio por Hertz em 1890. Os experimentos tornaram possível o estudo da ótica como ramo do eletromagnetismo, com as leis fundamentais das equações de Maxwell.

\subsubsection{Exemplo ilustrativo}

Considere um eletrodinamômetro Ampère-padrão.

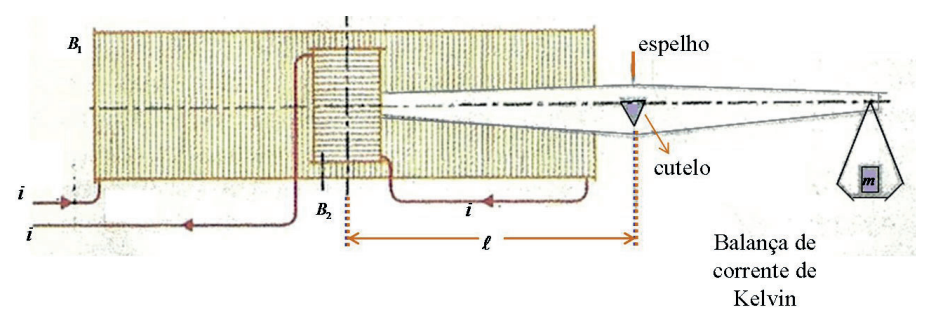

É constituído de uma bobina fixa $\mathrm{B}_{1}$ e uma bobina móvel $\mathrm{B}_{2}$ na outra extremidade do travessão. $\mathrm{O}$ campo produzido pela corrente $i$ no seu interior não é uniforme, mas proporcional a $i$. O conjugado $m \ell$ que tira o travessão da balança da horizontal é proporcional a $i^{2}$.

Coloca-se um contrapeso $m$ no prato da balança para fazer o travessão voltar à posição horizontal. ${ }^{55}$

A equação de equilíbrio será: $a i^{2}=m g \ell$

\footnotetext{
${ }_{55}$ Para facilitar esse trabalho, usa-se um espelho sobre o travessão, na posição sobre o cutelo. Um raio de luz incide no espelho e seu reflexo indica a posição de equilíbrio.
} 
Logo: $i=\sqrt{\frac{g \ell}{a}} \sqrt{m}$ em que $a$ é uma constante característica do instrumento.

É determinado fazendo-se passar a mesma corrente no aparelho e em um eletrodinamômetro absoluto.

Após graduado, o aparelho permite medir $i$ com uma precisão de 1/10000, e pode ser utilizado nos laboratórios para a verificação de amperímetros.

O eletrodinamômetro absoluto tem a bobina $B_{1}$ longa e larga, e a menor, $B_{2}$, é colocada no campo aproximadamente uniforme, produzida pela maior $B_{1}$.

Sendo $n$ o número de espiras por cm de $B_{1}, N$ é o total de espiras da menor $B_{2} . S$ é a superfície em $\mathrm{cm}^{2}$ da menor e $a$ é o termo corretivo pelo comprimento finito de $B_{1}$. Igualando os conjugados, teremos: $4 \pi N S(1-a) i^{2} 10^{-2}=m g \ell$

Daqui explicitamos i: como se despreza a influência do campo terrestre sobre $B_{2}$, pode-se eliminar sua influência invertendo o sentido da corrente na bobina fixa, sem mudar na móvel.

A massa necessária para restabelecer o equilíbrio será $2 m$, pois a ação da Terra é a mesma em ambos os casos e a ação da bobina fixa é trocar de sinal.

O eletrodinamômetro Ampère-padrão tem a bobina $B_{1}$ muito curta

(LAROUSSE, LEMOINE - GUYOT).

Definido o Ampère, podemos determinar o Coulomb como a quantidade de carga que flui através de qualquer secção transversal de um condutor em 1 segundo, quando a corrente é de 1 Ampère.

\subsection{TENSOR DO CAMPO ELETROMAGNÉTICO}

\subsubsection{Quadripotencial}

Vimos na Seção 5.5 que $\overrightarrow{\mathrm{F}} / \mathrm{q}=\overrightarrow{\mathrm{E}}$, campo elétrico, e na Seção 5.17.1, $\overrightarrow{\mathrm{E}}=-$ gradV, sendo V o potencial elétrico. Chamemos $\mathrm{V}$ de $\varphi$, então teremos: $\overrightarrow{\mathrm{E}}=-\operatorname{grad} \varphi$, em notação tensorial: $\mathrm{E}_{\mathrm{i}} \overrightarrow{\mathrm{e}}_{\mathrm{i}}=$ $-\left(\partial \varphi / \partial \mathrm{x}_{\mathrm{i}}\right) \overrightarrow{\mathrm{e}}_{\mathrm{i}}$. Nota-se que o gradiente sempre terá as suas componentes iguais à derivada em relação às correspondentes coordenadas.

As propriedades do campo elétrico são caracterizadas por um quadrivetor $A_{i}$, chamado quadripotencial, cujas componentes são funções das coordenadas e do tempo. Expressamos sua "Ação" por meio do termo: $S=\frac{q}{c} \int_{a}^{b} A_{i} d x_{i}$. (a) 
De acordo com a definição da função "Ação", apresentada no Anexo 3, equação 2: $S=\int_{\mathrm{x}_{1}}^{\mathrm{x}_{2}} \int_{\mathrm{t}_{1}}^{\mathrm{t}_{2}} \mathrm{~F} \mathrm{dtdx}$, sabemos que $\mathrm{F}=\mathrm{qE} \quad$ e $\mathrm{c}=\mathrm{d} \tau / \mathrm{dt} \quad \therefore \mathrm{dt}=\mathrm{d} \tau / \mathrm{c}$, substituindo, obtemos: $\mathrm{S}=\int_{\mathrm{x}_{1}}^{\mathrm{x}_{2}} \int_{\tau_{1}}^{\tau_{2}} \frac{\mathrm{qE}_{\mathrm{i}}}{\mathrm{c}} \mathrm{d} \tau \mathrm{d} \mathrm{x}_{\mathrm{i}}, \quad \overrightarrow{\mathrm{E}}=$ $-\operatorname{grad} \varphi, \log \mathrm{E}_{\mathrm{i}}=-\partial \varphi / \partial \mathrm{x}_{\mathrm{i}} \Rightarrow \mathrm{d} \varphi=-\mathrm{E}_{\mathrm{i}} \mathrm{dx}_{\mathrm{i}}$

$$
\therefore \varphi=-\int_{\mathrm{a}}^{\mathrm{b}} \mathrm{E}_{\mathrm{i}} \mathrm{dx}_{\mathrm{i}} \Rightarrow \mathrm{S}=-\frac{\mathrm{q}}{\mathrm{c}} \int_{\mathrm{a}}^{\mathrm{b}} \int_{\tau_{1}}^{\tau_{2}} \mathrm{E}_{\mathrm{i}} \mathrm{dx} \mathrm{x}_{\mathrm{i}} \mathrm{d} \tau
$$

Então: $A_{i}=-\int_{a}^{b} E_{i} d_{x_{i}}$ e assumindo $\tau=x_{4}$, no sistema de coordenadas quadridimensional.

$\mathrm{S}=\frac{\mathrm{q}}{\mathrm{c}} \int_{\mathrm{a}}^{\mathrm{b}} \mathrm{A}_{\mathrm{i}} \mathrm{dx}_{\mathrm{i}}$, isto é, $\mathrm{A}_{\mathrm{i}}$ tem dimensões de um potencial, considerado um potencial mais genérico, utilizado no sistema de coordenadas quadridimensional, por isso, é chamado de Quadripotencial.

As três componentes espaciais do quadrivetor $A_{i}$ formam um vetor tridimensional $\vec{A}$, chamado "vetor potencial" do campo. A componente temporal do quadrivetor $A_{i}$ é imaginária e descrita como $A_{4}=i \varphi$.

O real $\varphi$ é chamado “potencial escalar” do campo:

$$
\mathrm{A}_{1,2,3}=\mathrm{A}_{\mathrm{x}, \mathrm{y}, \mathrm{z}} \quad \mathrm{A}_{4}=\mathrm{i} \varphi
$$

Como consequência, temos: $S=\int_{a}^{b}\left(\frac{q}{c} \vec{A} \cdot d \vec{r}-q \varphi d t\right)=\int_{t_{1}}^{t_{2}}\left(\frac{q}{c} \vec{A} \cdot \vec{v}-q \varphi\right) d t(b), d \vec{r}=\vec{v} d t$ e d $\tau=c d t$

Então, a energia será: $L=\frac{q}{c} \vec{A} \cdot \vec{v}-q \varphi$, pois $S=\int L d t$, ver Anexo 3, equação 2 .

Derivando em $\overrightarrow{\mathrm{r}}: \frac{\partial \mathrm{L}}{\partial \overrightarrow{\mathrm{r}}}=\vec{\nabla} \mathrm{L}=\frac{\mathrm{q}}{\mathrm{c}} \operatorname{grad}(\overrightarrow{\mathrm{A}} \cdot \overrightarrow{\mathrm{v}})-\mathrm{q} \operatorname{grad} \varphi \quad{ }^{57}$

Como: $\operatorname{grad}(\vec{a} \cdot \vec{b})=(\vec{a} \cdot \vec{\nabla}) \vec{b}+(\vec{b} \cdot \vec{\nabla}) \vec{a}+\vec{b} \times \operatorname{rot} \vec{a}+\vec{a} \times \operatorname{rot} \vec{b}$

Deriva-se em relação a $\vec{r}$, supondo $\vec{v}$ cte:

$$
\frac{\partial \mathrm{L}}{\partial \overrightarrow{\mathrm{r}}}=\frac{\mathrm{q}}{\mathrm{c}}(\overrightarrow{\mathrm{v}} \cdot \vec{\nabla}) \overrightarrow{\mathrm{A}}+\frac{\mathrm{q}}{\mathrm{c}} \overrightarrow{\mathrm{V}} \times \operatorname{rot} \overrightarrow{\mathrm{A}}-\mathrm{q} \operatorname{grad} \varphi
$$

A “Ação”, como visto no Anexo 3, é $S=\int_{t_{1}}^{t_{2}} L d t=\int_{x_{1}}^{x_{2}} p d x$

Em um sistema de referências inercial relacionado a coordenadas cartesianas:

$$
\mathrm{ds}^{2}=\mathrm{c}^{2} \mathrm{dt}^{2}-\mathrm{dx}^{2}-\mathrm{dy}^{2}-\mathrm{dz}^{2} \Rightarrow-\mathrm{ds}^{2}=\mathrm{dx}^{2}+\mathrm{dy}^{2}+\mathrm{dz}^{2}-\mathrm{c}^{2} \mathrm{dt}^{2}
$$

Fazendo: $\mathrm{d} \tau=\mathrm{icdt}$, obtemos: $-\mathrm{ds}^{2}=\mathrm{dx}^{2}+\mathrm{dy}^{2}+\mathrm{dz}^{2}+\mathrm{d} \tau^{2}$, ou seja, $-\mathrm{ds}^{2}$ é a resultante dos quatro infinitésimos: $\mathrm{dx}, \mathrm{dy}, \mathrm{dz}$ e $\mathrm{d} \tau$ no sistema de coordenadas tetradimensional, de uma forma semelhante ao obtido por meio do Teorema de Pitágoras para espaço tridimensional. Como ds tem características similares a $d x, d y, d z$ e $d \tau$, é possível expressar $S$ como: $S=-\alpha \int_{a}^{b} d s$, sendo $\alpha$ uma constante a determinar. Recorde-se que: $S=\int_{a}^{b}\left(\frac{q}{c} \vec{A} \cdot d \vec{r}-q \varphi d t\right)=\int_{t_{1}}^{t_{2}}\left(\frac{q}{c} \vec{A} \cdot \vec{v}-q \varphi\right) d t(b), d \vec{r}=\vec{v} d t$ e $\mathrm{d} \tau=\mathrm{cdt}$ ver equação 1 .

\footnotetext{
${ }^{56}$ Curiosamente, trata-se de um tensor misto de três componentes de vetor e um componente de escalar.

${ }^{57}$ É um vetor cujas componentes são as derivadas de L em relação às componentes do vetor $\overrightarrow{\mathrm{r}}$.
} 
Na Seção 7.2, equação 2 determinamos que ds, será: ds $=\mathrm{cdt} \sqrt{1-\mathrm{v}^{2} / \mathrm{c}^{2}}(1 \mathrm{a})$, que fornece a relação entre ds e dt. ${ }^{58}$

Comparando com a Equação (1), a lagrangeana $L$ será expressa como: $L=-\alpha c \sqrt{1-v^{2} / c^{2}}$. No caso mais simples, a lagrangeana será formada com a energia cinética: $L=1 / 2 \mathrm{~m}_{0} \mathrm{v}^{2}$

Desenvolvendo $\left(1-\mathrm{v}^{2} / \mathrm{c}^{2}\right)^{1 / 2}$ como uma série de Maclaurin: $(1+\mathrm{x})^{\mathrm{k}}=1+\mathrm{kx}+\cdots$, pelo Binômio de Newton quando $\mathrm{k}$ for fracionário, isto é, não inteiro. $|\mathrm{x}|<1$ (GRANVILLE). (3)

Obtemos: $\left(1-v^{2} / c^{2}\right)^{1 / 2} \simeq 1-v^{2} / 2 c^{2} \therefore L \simeq-\alpha c+\frac{\alpha v^{2}}{2 c}$. Como $S, L$ deve ser integrado, e o termo constante $\alpha$ c desaparece na integração definida. Comparando com a Equação (2), $\alpha=\mathrm{m}_{0}$ c, constantes que tem dimensão de quantidade de movimento. $\therefore \mathrm{L}=-\mathrm{m}_{0} \mathrm{c}^{2}+\frac{\mathrm{mv}^{2}}{2}$, sendo $\mathrm{m}_{0} \mathrm{c}^{2}$ energia de repouso, para $\mathrm{v}=0$. Com isto, $\mathrm{S}=-\mathrm{m}_{0} \mathrm{c} \int_{\mathrm{a}}^{\mathrm{b}} \mathrm{ds}$

Adicionando-se a "Ação" devida à partícula material: $\mathrm{S}=-\mathrm{mc} \int_{\mathrm{a}}^{\mathrm{b}}\left(\mathrm{ds}+\frac{\mathrm{q}}{\mathrm{c}} \mathrm{A}_{\mathrm{i}} \mathrm{dx} \mathrm{x}_{\mathrm{i}}\right)$, fazendo: $\mathrm{m}_{0}=\mathrm{m}$, ver também Equações (a) e (b).

Obtemos: $\mathrm{L}=-\mathrm{mc}^{2} \sqrt{1-\mathrm{v}^{2} / \mathrm{c}^{2}}+\frac{\mathrm{q}}{\mathrm{c}} \overrightarrow{\mathrm{A}} \cdot \overrightarrow{\mathrm{v}}-\mathrm{q} \varphi \quad(3 \mathrm{a} 1)$

Derivando em $\vec{v}$, temos a quantidade de movimento, conforme Anexo 3, equação $2 \mathrm{a}, \overrightarrow{\mathrm{p}}=\partial \mathrm{L} / \partial \overrightarrow{\mathrm{v}}$.

Na Física clássica, seria $m \vec{v}$, generalizada aqui como:

$\frac{\partial \mathrm{L}}{\partial \overrightarrow{\mathrm{v}}}=\frac{\mathrm{m} \overrightarrow{\mathrm{v}}}{\sqrt{1-\mathrm{v}^{2} / \mathrm{c}^{2}}}+\frac{\mathrm{q}}{\mathrm{c}} \overrightarrow{\mathrm{A}}=\overrightarrow{\mathrm{p}}+\frac{\mathrm{q}}{\mathrm{c}} \overrightarrow{\mathrm{A}}$, quando $\mathrm{v} \ll \mathrm{c}, \mathrm{m \vec {v }} / \sqrt{1-\mathrm{v}^{2} / \mathrm{c}^{2}} \simeq \overrightarrow{\mathrm{p}}$ da Física clássica. O $1^{\circ}$ termo é espacial e o $2^{\circ}$, temporal.

Como: $\frac{\mathrm{d}}{\mathrm{dt}} \frac{\partial \mathrm{L}}{\partial \overrightarrow{\mathrm{v}}}=\frac{\mathrm{d}}{\mathrm{dt}} \frac{\partial \mathrm{L}}{\partial(\mathrm{d} \overrightarrow{\mathrm{r}} / \mathrm{dt})}=\frac{\partial \mathrm{L}}{\partial \overrightarrow{\mathrm{r}}}$

Teremos: $\frac{d}{d t}\left(\vec{p}+\frac{q}{c} \vec{A}\right)=\frac{q}{c}(\vec{v} \cdot \vec{\nabla}) \vec{A}+\frac{q}{c} \vec{v} \times \operatorname{rot} \vec{A}-q \operatorname{grad} \varphi=\frac{\partial L}{\partial \vec{r}}$

A derivada substancial: $\frac{d \vec{A}}{d t}=\frac{\partial \vec{A}}{\partial t}+(\vec{v} \cdot \vec{\nabla}) \vec{A}$, ver Shames e Borisenko.

A derivada total $d \vec{A} / d t$ é composta da variação do vetor potencial em relação ao tempo: $\partial \vec{A} / \partial t$ no ponto dado do espaço, mais a variação quando se passa de um ponto do espaço a outro, que se encontra à distância dr, cuja variação no tempo será: $[(\mathrm{d} \overrightarrow{\mathrm{r}} / \mathrm{dt}) \cdot \vec{\nabla}] \overrightarrow{\mathrm{A}}$.

Outra forma mais simples de resolver matematicamente por cálculo vetorial e diferencial é com a utilização da regra de cadeia. Considerando que: $\vec{A}=\vec{A}(x, y, z, t)$, e também:

${ }^{58} \frac{\mathrm{ds}}{\mathrm{c}}=\mathrm{dt} \sqrt{1-\frac{\mathrm{dx}^{2}+\mathrm{dy}^{2}+\mathrm{dz}^{2}}{\mathrm{c}^{2} \mathrm{dt}^{2}}}$, como: $\mathrm{v}^{2}=\frac{\mathrm{dx}^{2}+\mathrm{dy}^{2}+\mathrm{dz}^{2}}{\mathrm{dt}^{2}}$, obtemos a expressão anterior. 


$$
\begin{aligned}
& v_{x}=f[x(t), y(t), z(t), t] \\
& v_{y}=g[x(t), y(t), z(t), t] \\
& v_{z}=h[x(t), y(t), z(t), t]
\end{aligned}
$$

$\frac{d \vec{A}}{d t}=\left(\frac{\partial \vec{A}}{\partial x} \frac{\partial x}{\partial t}+\frac{\partial \vec{A}}{\partial y} \frac{\partial y}{\partial t}+\frac{\partial \vec{A}}{\partial z} \frac{\partial z}{\partial t}\right)+\frac{\partial \vec{A}}{\partial t}=\left(v_{x} \frac{\partial \vec{A}}{\partial x}+v_{y} \frac{\partial \vec{A}}{\partial y}+v_{z} \frac{\partial \vec{A}}{\partial z}\right)+\frac{\partial \vec{A}}{\partial t}=(\vec{v} \cdot \vec{\nabla}) \vec{A}+\frac{\partial \vec{A}}{\partial t}$, ou tensorialmente: $\overrightarrow{\mathrm{V}} \cdot \vec{\nabla} \Upsilon \overrightarrow{\mathrm{A}}+\frac{\partial \overrightarrow{\mathrm{A}}}{\partial \mathrm{t}}$ sendo $\mathcal{X}$, produto diádico.

Então: $\vec{F}=\frac{d \vec{p}}{d t}=-\frac{q}{c} \frac{\partial \vec{A}}{\partial t}-q \operatorname{grad} \varphi+\frac{q}{c} \vec{v} \times \operatorname{rot} \vec{A}$, obtida substituindo $d \vec{p} / d t$ na Equação 3a e, em consequência, elimina-se o termo $(\overrightarrow{\mathrm{v}} \cdot \vec{\nabla}) \overrightarrow{\mathrm{A}}$.

Temos aqui duas parcelas:

Vetor "Campo elétrico": $\vec{E}=-\frac{1}{c} \frac{\partial \vec{A}}{\partial t}-\operatorname{grad} \varphi$, diferentemente do caso acima, que se referia a $\vec{E}$ constante no tempo. Em consequência, $\vec{A}$ também.

A $2^{\mathrm{a}}$ parcela é o vetor "Campo magnético": $\overrightarrow{\mathrm{H}}=\operatorname{rot} \overrightarrow{\mathrm{A}}$, para $\mathrm{i}, \mathrm{j}, \mathrm{k}=1,2,3$.

Então, temos: $\vec{F}=\frac{d \vec{p}}{d t}=q \vec{E}+\frac{q}{c} \vec{v} \times \vec{H}=q \vec{E}+q \vec{v} \times \vec{B}$, pois $\vec{H}=\vec{B} c$

Como será visto na Seção 5.26, Equação (5.9). Essa expressão é a força de Lorentz, como visto na Seção 5.11, Equação (2).

Chamando $\mathrm{X}, \mathrm{Y}$ e Z as componentes do campo elétrico $\vec{E}$, e considerando o espaço vazio, isto é, sem matéria, obtemos pela lei de Gauss para eletricidade ou $1^{\text {a }}$ lei de Maxwell, ver Seção 5.10, Equação (2): $\frac{\rho}{\varepsilon_{0}}=0$

div $\vec{E}=0$ Logo: $\frac{\partial \mathrm{x}}{\partial \mathrm{x}}+\frac{\partial \mathrm{Y}}{\partial \mathrm{y}}+\frac{\partial \mathrm{Z}}{\partial \mathrm{z}}=0$, pois, se não houver material e ausência de carga: $\rho=0$ e $\vec{\nabla} \cdot \overrightarrow{\mathrm{E}}=$

Chamando $L, M$ e $N$ as componentes da indução magnética $\vec{B}$, obteremos, conforme o final da

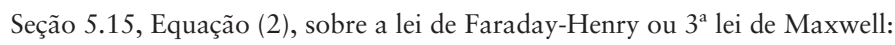

$$
\begin{aligned}
\operatorname{rot} \vec{E}=-\frac{\partial \vec{B}}{\partial t}, \quad \text { ou seja: } & \frac{\partial Z}{\partial y}-\frac{\partial Y}{\partial z}=-\frac{\partial L}{\partial t} \\
\frac{\partial X}{\partial z}-\frac{\partial Z}{\partial x}= & =\frac{\partial M}{\partial t} \\
\frac{\partial Y}{\partial x}-\frac{\partial X}{\partial y} & =-\frac{\partial N}{\partial t}
\end{aligned}
$$

Conforme vimos na Seção 5.12 sobre a lei de Gauss para o magnetismo, ou 2a lei de Maxwell, Equação (1), o div $\vec{B}=0$ se anula e: 


$$
\frac{\partial L}{\partial x}+\frac{\partial M}{\partial y}+\frac{\partial N}{\partial z}=0
$$

Levando em conta o espaço vazio, isto é, $\rho=0$

Sabemos que a velocidade da luz c é cerca de $300.000 \mathrm{~km} / \mathrm{s}$; vamos atribuir o valor 1 para $\mathcal{C}$. Como $c^{2}=1 / \varepsilon_{0} \mu_{0}$, no final da Seção 5.23 , consequentemente $\varepsilon_{0} \mu_{0}=1$ e, do final da Seção $5.16,{ }^{59}$ sobre a lei de Ampère-Maxwell, ou $4^{a}$ lei de Maxwell, Equação (5):

$$
\begin{aligned}
& \operatorname{rot} \vec{B}=\frac{\partial \vec{E}}{\partial t} \text { então: } \frac{\partial N}{\partial y}-\frac{\partial M}{\partial z}=\frac{\partial X}{\partial t} \\
& \text { Pois } \rho=0 \frac{\partial L}{\partial z}-\frac{\partial N}{\partial x}=\frac{\partial Y}{\partial t} \\
& \frac{\partial M}{\partial x}-\frac{\partial L}{\partial y}=\frac{\partial Z}{\partial t}
\end{aligned}
$$

Usando o índice conotando derivada em relação à correspondente variável independente, por exemplo: $N_{y}=\frac{\partial N}{\partial y}$, podemos escrever:

$$
\begin{aligned}
& \mathrm{N}_{\mathrm{y}}-\mathrm{M}_{\mathrm{z}}-\mathrm{X}_{\mathrm{t}}=0 \\
& \mathrm{~L}_{\mathrm{z}}-\mathrm{N}_{\mathrm{x}}-\mathrm{Y}_{\mathrm{t}}=0 \\
& \mathrm{M}_{\mathrm{x}}-\mathrm{L}_{\mathrm{y}}-\mathrm{Z}_{\mathrm{t}}=0 \\
& \mathrm{X}_{\mathrm{x}}+\mathrm{Y}_{\mathrm{y}}+\mathrm{Z}_{\mathrm{z}}=0
\end{aligned}
$$

$$
\begin{aligned}
& \mathrm{Z}_{\mathrm{y}}-\mathrm{Y}_{\mathrm{z}}+\mathrm{L}_{\mathrm{t}}=0 \\
& \mathrm{X}_{\mathrm{z}}-\mathrm{Z}_{\mathrm{x}}+\mathrm{M}_{\mathrm{t}}=0 \\
& \mathrm{Y}_{\mathrm{x}}-\mathrm{X}_{\mathrm{y}}+\mathrm{N}_{\mathrm{t}}=0 \\
& \mathrm{~L}_{\mathrm{x}}+\mathrm{M}_{\mathrm{y}}+\mathrm{N}_{\mathrm{z}}=0
\end{aligned}
$$

\subsubsection{Distribuição da matéria}

Podemos considerar a matéria distribuída continuamente no espaço ou a teoria discreta na qual a matéria consiste em pontos materiais, cada um dos quais carrega uma massa finita. A teoria contínua considera a matéria distribuída continuamente através do espaço. Cada uma delas é o caso limite da outra.

Podemos iniciar com pontos materiais, depois aumentar sua quantidade e, ao mesmo tempo, diminuir a massa de cada um, assim, aproximar com algum grau de precisão de uma dada distribuição contínua; ou podemos iniciar com uma distribuição contínua e depois fazer a densidade diminuir em toda

\footnotetext{
59 Fazendo a relação: velocidade/velocidade da luz $\beta=v / c$, quando afirmarmos que $\beta=0,01$, significa que a velocidade é 1 centésimo ( $1 / 100$ ) da velocidade da luz, isto é, a velocidade relativa em relação à velocidade da luz, e veremos a importância de se tomar a velocidade da luz no vácuo como referência.
} 
parte, exceto nas vizinhanças constantemente decrescentes de um número discreto de pontos e, assim, aproximar, com qualquer precisão, uma certa distribuição discreta.

$\mathrm{Na}$ teoria discreta da matéria, as equações apresentadas anteriormente ainda valem em toda parte, exceto nos pontos ocupados pela matéria, que aparece como singularidade do campo, e algumas características da matéria, como a carga elétrica, aparecem como resíduos correspondentes a essas singularidades. Estes últimos são análogos às singularidades e resíduos da teoria das funções analíticas de uma variável complexa.

Levando em conta a lei de Gauss para eletricidade e a lei de Ampère-Maxwell [Seção 5.10 e final da Seção 5.16, Equações (2) e (5)], e colocando na forma gaussiana (sistema cgs, Seção 2.3), na presença de carga elétrica:

$$
\operatorname{div} \overrightarrow{\mathrm{E}}=4 \pi \rho, \text { ou seja: } \frac{\partial X}{\partial x}+\frac{\partial Y}{\partial y}+\frac{\partial Z}{\partial z}=4 \pi \rho
$$

$$
\operatorname{rot} \vec{B}=\frac{1}{c^{2}} \frac{\partial \vec{E}}{\partial t}+\frac{1}{c^{2}} 4 \pi \rho \vec{v}(5.5 b) \text {, isto é: } \quad N_{y}-M_{z}-X_{t}=4 \pi \rho u
$$

Sendo: $\varepsilon_{0}=1 / 4 \pi$

$$
\mathrm{L}_{\mathrm{z}}-\mathrm{N}_{\mathrm{x}}-\mathrm{Y}_{\mathrm{t}}=4 \pi \rho \mathrm{v}
$$

$$
\mu_{0}={\frac{4 \pi}{c^{2}}}^{(5.5 a)}
$$

$$
\mathrm{Mx}_{\mathrm{x}}-\mathrm{L}_{\mathrm{y}}-\mathrm{Z}_{\mathrm{t}}=4 \pi \rho \mathrm{w}
$$

Sendo $\mathrm{u}, \mathrm{v}, \mathrm{w}$ componentes de $\overrightarrow{\mathrm{v}}$ e lembrando que $\mathrm{c}^{2}=1$.

No sistema cgs, no caso da indução magnética $\vec{B}$, o sistema de equações não muda:

$$
\begin{aligned}
& Z_{y}-Y_{z}+L_{t}=0 \\
& X_{z}-Z_{x}+M_{t}=0 \\
& Y_{x}-X_{y}+N_{t}=0 \\
& L_{x}+M_{y}+N_{z}=0
\end{aligned}
$$

Temos quatro variáveis independentes $\mathrm{x}, \mathrm{y}, \mathrm{z}, \mathrm{t}$, e seis quantidades componentes dos vetores $\overrightarrow{\mathrm{E}}$ e $\overrightarrow{\mathrm{B}}$ : $\mathrm{X}, \mathrm{Y}, \mathrm{Z}, \mathrm{L}, \mathrm{M}, \mathrm{N}$. Um vetor tem poucas componentes para solucionar a questão. Em vez de usar dois vetores, Minkowski propôs, em 1907, utilizar um tensor de ordem 2. Naturalmente, um tensor tem muitas componentes, em nosso caso, 16: 4 na diagonal principal, 6 acima da diagonal e 6 abaixo. ${ }^{60}$ Fazemos, então os da diagonal principal nulos e os abaixo da diagonal principal com sinal oposto aos de cima. Assim, fica-se com 6 componentes X, Y, Z, L, M, N. Essa restrição é expressa por:

$$
\mathrm{F}_{\mathrm{ij}}+\mathrm{F}_{\mathrm{ji}}=0 \quad \mathrm{~F}_{\mathrm{ij}}=-\mathrm{F}_{\mathrm{ji}} \quad \text { Tensor antissimétrico }^{61}
$$

\footnotetext{
${ }^{60}$ Ver o conceito de tensor na Seção 5.3.

${ }_{61}^{61} F_{i k}=\frac{\partial A_{k}}{\partial x_{i}}-\frac{\partial A_{i}}{\partial x_{k}}(\mathrm{i}, \mathrm{k}=1,2,3,4) \quad A_{i}=$ potencial vetorial para $\mathrm{i}=1,2,3$ e $\mathrm{A}_{4}=\mathrm{i} \varphi$ $\overrightarrow{\mathrm{H}}=\overrightarrow{\mathrm{B}} \mathrm{c}=\operatorname{rot} \overrightarrow{\mathrm{A}}$, ver Quadripotencial seção 5.25.1, Equação (4).
} 
$F_{\mathrm{ij}}$ é chamado "tensor do campo eletromagnético".

De fato, se $i=j: \quad F_{i i}+F_{i i}=0$, ou seja: $\mathrm{F}_{\mathrm{ii}}=0$

$F_{i i}$ são os elementos da diagonal principal.

$$
\begin{array}{rlrl}
\text { Teremos: } X=F_{41} & Y=F_{42} & Z=F_{43} \\
L=F_{23} & M=F_{31} & N=F_{12}
\end{array}
$$

Para simplificar, usamos para a derivada a notação de Cauchy: $\quad \partial_{i} \psi=\frac{\partial \psi}{\partial x_{i}}$

Observando que $F_{14}=-X$, podemos escrever (5.3) na seguinte forma:

$$
\begin{aligned}
& \partial_{2} F_{12}+\partial_{3} F_{13}+\partial_{4} F_{14}=0 \\
& \partial_{3} F_{23}+\partial_{1} F_{21}+\partial_{4} F_{24}=0 \\
& \partial_{1} F_{31}+\partial_{2} F_{32}+\partial_{4} F_{34}=0 \\
& \partial_{1} F_{41}+\partial_{2} F_{42}+\partial_{3} F_{43}=0
\end{aligned}
$$

Simbolicamente: $\partial_{\mathrm{i}} \mathrm{F}_{\mathrm{ji}}=0$

O sinal negativo no $2^{\circ}$ membro das Equações em (5.1) ocasiona dificuldades para formar um sistema simétrico ao que vimos no sistema de Equações (5.2). Para superá-lo, usamos o indicado por Poincaré e Marcolongo, e prenunciado por Hamilton desde 1845, ou seja, quantidades imaginárias ao lado de reais. Se soubermos as regras formais de operação, não haverá dificuldades, pois a nova notação é de natureza inteiramente formal. Teremos, então:

$$
\begin{array}{llll}
x=x_{1} & y=x_{2} & z=x_{3} & c t i=x_{4}(5.6 \mathrm{~b}) \text { lembremos } c=1 \\
i X=F_{41} & i Y=F_{42} & i Z=F_{43} & \therefore t i=x_{4} \quad \text { (5.6a) } \\
L=F_{23} & M=F_{31} & N=F_{12} &
\end{array}
$$

O sistema de Equações (5.3) se transforma no sistema (5.6) como antes, e o sistema (5.4) se transforma em:

$$
\partial_{2} F_{43}+\partial_{3} F_{24}+\partial_{4} F_{32}=0
$$




$$
\begin{aligned}
& \partial_{1} F_{34}+\partial_{3} F_{41}+\partial_{4} F_{13}=0 \\
& \partial_{1} F_{42}+\partial_{2} F_{14}+\partial_{4} F_{21}=0 \\
& \partial_{1} F_{23}+\partial_{2} F_{31}+\partial_{3} F_{12}=0
\end{aligned}
$$

Simbolicamente: $\partial_{\mathrm{i}} \mathrm{F}_{\mathrm{jk}}+\partial_{\mathrm{j}} \mathrm{F}_{\mathrm{ki}}+\partial_{\mathrm{k}} \mathrm{F}_{\mathrm{ij}}=0$

Podemos arranjar os componentes do tensor em uma disposição quadrada:

$\left|\begin{array}{llll}\mathrm{F}_{11} & \mathrm{~F}_{12} & \mathrm{~F}_{13} & \mathrm{~F}_{14} \\ \mathrm{~F}_{21} & \mathrm{~F}_{22} & \mathrm{~F}_{23} & \mathrm{~F}_{24} \\ \mathrm{~F}_{31} & \mathrm{~F}_{32} & \mathrm{~F}_{33} & \mathrm{~F}_{34} \\ \mathrm{~F}_{41} & \mathrm{~F}_{42} & \mathrm{~F}_{43} & \mathrm{~F}_{44}\end{array}\right|$ ou seja: $\quad\left|\begin{array}{rrrr}\mathrm{O} & \mathrm{N} & -\mathrm{M} & -\mathrm{iX} \\ -\mathrm{N} & \mathrm{O} & \mathrm{L} & -\mathrm{iY} \\ \mathrm{M} & -\mathrm{L} & \mathrm{O} & -\mathrm{iZ} \\ \mathrm{iX} & \mathrm{iY} & \mathrm{iZ} & 0\end{array}\right|$

Como $u_{i}=\frac{\partial x_{i}}{\partial t} \quad u_{4}=i$ pois $x_{4}=t i \quad$ [ver Equação $\left.(5.6 \mathrm{a})\right]$

Considerando as equações de Maxwell com matéria (carga elétrica), (5.5) obtemos: $\partial_{\mathrm{i}} \mathrm{F}_{\mathrm{ji}}=4 \pi \rho \mathrm{u}_{\mathrm{j}}{ }^{62}$ $\mathrm{u}_{\mathrm{j}}$ são componentes do vetor velocidade $\vec{v}$.

\subsection{VETOR DE POYNTING}

Para entender o vetor de Poynting, devemos recapitular as energias do campo elétrico e magnético nas Seções 5.19 e 5.21, Equações (1) e (3), $\quad \mathrm{E}_{e}=\frac{1}{2} \frac{Q^{2}}{C} \quad$ e $\quad E_{B}=\frac{1}{2} L i^{2}$

A energia de um condensador carregado está associada ao campo elétrico do condensador e não às cargas nas placas.

A distribuição de energia por unidade de volume é chamada de “densidade de energia” $\mathrm{u}_{\mathrm{e}}$.

Seja um condensador de placas paralelas. Aplicando a lei de Gauss [Seção 5.10, Equações (1a) e (0)], no volume de controle tracejado:

$$
\int \vec{E} \cdot d \vec{A}=\frac{Q}{\varepsilon} \quad \Rightarrow \quad E \cos \theta d A=\frac{d Q}{\varepsilon} \quad \theta=0 \quad \cos \theta=1
$$

\footnotetext{
${ }^{62}$ Compara-se com o sistema de Equações 5.3, da qual resultou o sistema 5.5.
} 


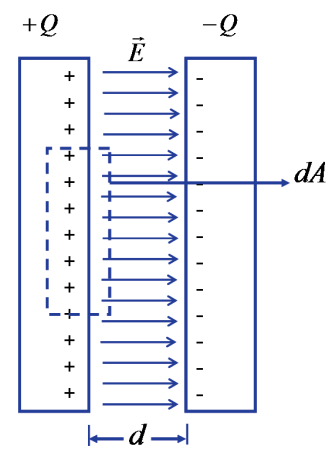

Fazendo $\sigma$ cargas por unidade de área, temos:

Cargas no volume de controle: $d Q=\sigma d A$

Logo: $E d A=\frac{1}{\varepsilon} \sigma d A \quad$ e $\quad E=\frac{1}{\varepsilon} \sigma$

$\mathrm{E}_{\mathrm{e}}=\frac{1}{2} \frac{\mathrm{Q}^{2}}{\mathrm{C}}$, Secção 5.19, equação 1 Como: $\Delta \mathrm{V}=\frac{\mathrm{Q}}{\mathrm{C}}$, Seção 5.18 .

$$
E_{e}=\frac{1}{2} C(\Delta V)^{2}=\frac{1}{2} Q \Delta V
$$

Densidade de energia elétrica: $u_{e}=\frac{\frac{1}{2} Q \Delta V}{A d} \quad A=$ área da placa, $d=$ distância entre as placas $\sigma=\frac{Q}{A}=\varepsilon E \quad$ campo elétrico [ver Seção 5.17.1, Equação (1b)]: $E=\frac{\Delta V}{d}$, substituímos em u $\mathrm{u}_{\mathrm{e}}$.

Portanto: $u_{e}=\frac{1}{2} \varepsilon E^{2}$

Foi deduzida para um campo uniforme, porém pode ser empregada para campo não uniforme, desde que se conheça $\vec{E}$ no ponto considerado.

Fazendo uma análise dimensional: $\mathrm{E}=\mathrm{F} / \mathrm{Q}$ e $\mathrm{F}=\mathrm{K}_{\mathrm{e}} \mathrm{Q}_{1} \mathrm{Q}_{2} / \mathrm{r}^{2}$, Seções 5.1 e 5.5 , $\varepsilon$ e $\mathrm{K}_{\mathrm{e}}$ são constantes de proporcionalidade.

$\left|u_{e}\right|=\frac{|F|}{|Q|} \frac{|Q|^{2}}{|Q| \cdot|L|^{2}}=\frac{|F| \cdot|L|}{|L|^{3}}$, energia por unidade de volume ou energia volumétrica.

Pois: $\frac{|F|}{|Q|}=\frac{|Q|^{2}}{|Q| \cdot|L|^{2}} \quad$ Pela lei de Coulomb: $F=\frac{1}{4 \pi \varepsilon_{0}} \frac{Q_{1} Q_{2}}{r^{2}}$ 


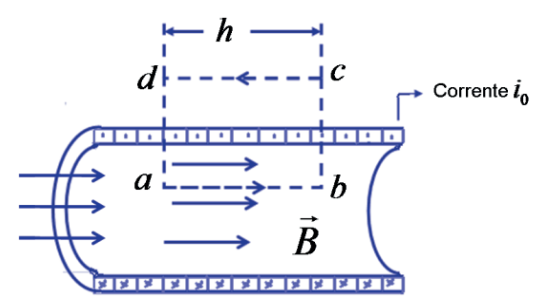

Tomamos um capacitor de placas paralelas como um protótipo para produzir um campo elétrico em certa região do espaço. De maneira similar podemos tomar uma pequena região do centro de um solenoide (bobina) longo como protótipo para produzir um campo magnético em certa região. Ver Figura no exemplo ilustrativo da Seção 5.24.1, sobre o Eletrodinamômetro Ampère padrão.

Aplicando a lei de Ampère, ver Seção 5.16, Equação (1): $\int_{c} \vec{B} \cdot \overrightarrow{d \ell}=\mu_{0} i \quad$ no circuito $a b c d a$, obtemos no trecho $a b$, como $\vec{B} \cdot d \vec{\ell}=B \cos \theta d \ell$ e $\theta=0, \int_{a}^{b} \vec{B} \cdot \overrightarrow{d \ell}=B h$

Nos trechos $b c$ e $d a$, como $\theta=90^{\circ}$, as integrais são nulas.

No trecho $c d$, a integral será nula, pois o campo $\vec{B}$ é praticamente nulo, fora da bobina.

Em consequência: $\int_{a b c d a} \vec{B} \cdot \overrightarrow{d \ell}=B h=\mu_{0} i$

Em cada espira, a corrente é $i_{0}$. Fazendo $n \mathrm{n}^{\circ}$ de espiras por unidade de comprimento do solenoide, em $h$ teremos $n h$ espiras. Logo: $B h=\mu_{0} i_{0} n h$ e $B=\mu_{0} i_{0} n$

Para simplificar, denotamos a corrente que passa em cada espira por $i$, escrevendo: $B=\mu_{0} i n$.

Da Seção 5.12, o fluxo magnético pela superfície $A$ delimitada pela parte interna de uma espira será: $\phi_{B}=\int \vec{B} \cdot \vec{n} d S=\int B \cos \theta d S \quad \operatorname{como} \quad \theta=0, \quad \cos \theta=1 \quad$ e $\quad \phi_{B}=B A$

Sendo $A=\int d S \quad B$ uniforme na secção A

A corrente em cada espira, sendo $i$, induz um fluxo magnético $\phi_{B}$. Havendo $N$ espiras, haverá um fluxo $N \phi_{B}$. A indutância $L$ será definida como: $L=\frac{N \phi_{B}}{i}$. Lembre-se da definição de autoindução na Seção 5.20 .

Logo, da expressão $B=\mu_{0}$ in, considerando $\ell$ um comprimento próximo do centro do solenoide, teremos: 
$L=\frac{N \phi_{B}}{i}=\frac{(n \ell)(B A)}{i}=\frac{(n \ell)\left(\mu_{0} i n\right)(A)}{i} \quad \therefore L / \ell=\mu_{0} n^{2} A$

Densidade de energia magnética: $u_{B}=\frac{1 / 2 L i^{2}}{A \ell}$. Tem as mesmas unidades que $u_{e}$.

Sendo $\frac{1}{2} L i^{2}$ a energia armazenada no volume $A \ell$, ver Seção 5.21, Equação (3).

Então: $u_{B}=\frac{L}{\ell} \frac{i^{2}}{2 A}$, substituindo pela expressão anterior para $L / \ell$ :

$u_{B}=1 / 2 \mu_{0} n^{2} i^{2} \quad$ Como $B=\mu_{0} i n$, teremos: $u_{B}=\frac{1}{2} \frac{B^{2}}{\mu_{0}}$

Pode-se mostrar que $u_{B}=\frac{1}{2} \frac{B^{2}}{\mu_{0}}=\frac{1}{2 \mu_{0} c^{2}} E^{2}=\frac{1}{2} \varepsilon_{0} E^{2}=u_{e}$

Tomando a equação da força de Lorentz, Seção 5.11.

$$
\vec{F}=\frac{d \vec{p}}{d t}=\frac{d}{d t}\left(m^{\prime} \vec{v}\right)=q(\vec{E}+\vec{v} \times \vec{B})
$$

Em que $\vec{p}$, quantidade de movimento $m^{\prime}$, massa relativística: $m^{\prime}=\frac{m}{\sqrt{1-\beta^{2}}}(5.8 \mathrm{~b})^{64}$

$\beta=\frac{v}{c} \quad c=$ velocidade da luz $\quad$ [Ver Seção 5.25, sobre Quadripotencial, Equação (3)].

Desenvolvendo pela série de Maclaurin, teremos:

$$
\frac{m}{\sqrt{1-\beta^{2}}}=m+\frac{1}{2} m \beta^{2}+\ldots \quad \text { pois: } \quad(1+\mathrm{x})^{\mathrm{n}}=1+\mathrm{nx}+\frac{\mathrm{n}(\mathrm{n}-1)}{1.2} \mathrm{x}^{2}+\ldots
$$

Para baixas velocidades $m^{\prime} \cong m \quad(v<<c)$ e podemos usar as fórmulas da mecânica clássica. Para velocidades próximas à da luz, é necessário fazer a correção relativística usando $\mathrm{m}^{\prime}$

(RAINICH, LANDAU, GRANVILLE).

${ }^{63}$ Pois $\vec{B}=\vec{E} / c$, ver Seção 5.23 , Equação $9, c=1 / \sqrt{\varepsilon_{0} \mu_{0}}$, ver equação 5 .

${ }^{64}$ Ver Seção 7.1, Equação (0), e Seção 7.4, Equação (1), em que será deduzido o fator de Lorentz que relaciona a massa relativística $m^{\prime}$ com a massa de repouso $m: \gamma=\frac{1}{\sqrt{1-\beta^{2}}}$, fator de Lorentz (5.8b). 
Retornando às densidades de energia, a densidade de energia total será: $u_{t}=u_{e}+u_{B}$.

Foram deduzidas considerando o espaço onde atua vazio, sem material, ou seja, sem cargas elétricas. Se estas existirem, as cargas elétricas ficam sujeitas à força de Lorentz [Seção 5.11, Equação (2)].

Pode parecer contraditório somar $\mathrm{u}_{\mathrm{e}}+\mathrm{u}_{\mathrm{B}}$ e mantê-la constante, tendo em vista Equação (1a) da Seção 5.22, mas devemos lembrar que, $i$ e q não são constantes, pois variam de zero a um valor máximo $\mathrm{i}_{\max }$ e $\mathrm{q}_{\max }$. Assim, quando $i$ for zero, q será máximo e igual a $\mathrm{q}_{\max } \mathrm{e}$ vice-versa; quando $\mathrm{q}$ for zero, $i$ será máximo e igual a $\mathrm{i}_{\max }$. Nos outros casos, terão valores intermediários, mantendo $\mathrm{u}_{\mathrm{t}}$ constante.

Da mesma forma para $E$ e $B$, que são variáveis oscilando entre 0 e $\mathrm{E}_{\max }$ e $\mathrm{B}_{\max }$, conforme as fórmulas (5.7) e (5.8), da Seção 5.26.

Isso também vale, naturalmente, para manter $E_{t}$ constante, conforme Equação (1a), Seção 5.22.

Como afirmado, vamos utilizar a equação da força de Lorentz, mas em lugar de q usaremos $\rho$, que é $\rho=\frac{d q}{d V}$, isto é, carga por unidade de volume, obtendo em lugar de $\vec{F}$, força por unidade de volume $\overrightarrow{\mathrm{f}}$ : $\vec{f}=\rho \vec{E}+\rho \vec{v} \times \vec{B}$, a força volumétrica.

Se as cargas contidas no volume $d V$ se deslocarem de uma distância $d \vec{r}$ no tempo $d t$, o trabalho feito pelo campo eletromagnético será: $\vec{f} \cdot d \vec{r}=\vec{f} \cdot \frac{d \vec{r}}{d t} d t=\vec{f} \cdot \vec{v} d t=[\rho \vec{E}+\rho \vec{v} \times \vec{B}] \cdot \vec{v} d t$

Esse trabalho provoca a variação da energia cinética du $\mathrm{u}_{\text {cin }}$ das cargas contidas no elemento $d V$ : $d u_{\text {cin }}=\vec{f} \cdot d \vec{r}$

O triplo produto escalar:

$$
\vec{v} \times \vec{B} \cdot \vec{v}=\left|\begin{array}{lll}
\mathrm{v}_{1} & \mathrm{v}_{2} & \mathrm{v}_{3} \\
\mathrm{~B}_{1} & \mathrm{~B}_{2} & \mathrm{~B}_{3} \\
\mathrm{v}_{1} & \mathrm{v}_{2} & \mathrm{v}_{3}
\end{array}\right|=0 \quad \text { (5.8a 1a) }
$$

A matriz é nula, pois tem duas linhas iguais.

Também podemos observar que o volume do paralelepípedo formado pelos três vetores $\vec{v}, \vec{B}$ e $\vec{v}$, que representa o triplo produto escalar, é nulo, tem dois vetores iguais $\overrightarrow{\mathrm{v}}$. Assim, como verificamos que $\rho \vec{v} \times \vec{B}$ é o vetor força magnética por unidade de volume $\overrightarrow{\mathrm{f}}_{\mathrm{m}}$, perpendicular a $\overrightarrow{\mathrm{v}}$, logo $\overrightarrow{\mathrm{f}}_{\mathrm{m}} \cdot \overrightarrow{\mathrm{v}}=0$ (5.8a1b).

Pois $\mathrm{f}_{\mathrm{m}} \cdot \mathrm{v} \cos \theta=0$, já que $\theta=90^{\circ}$ e $\cos 90^{\circ}=0$. Dessa forma, o vetor $\vec{f}_{e}=\rho \vec{E}$ será denominado vetor força elétrica por unidade de volume, cujo produto escalar $\mathrm{f}_{\mathrm{e}} \cdot \overrightarrow{\mathrm{v}} \mathrm{dt}$, em geral, não se anula.

Em consequência: $d u_{c i n}=\rho \vec{v} \cdot \vec{E} d t$ 
Portanto, a potência cinética será: $\frac{d u_{c i n}}{d t}=\rho \vec{v} \cdot \vec{E}$

Chamando de $P_{c}$ a perda de potência no circuito indutância, capacitância, resistência, LCR, ver equação $1 \mathrm{~d} 1$, seç̧ão 5.22

Recordemos a potência $P_{c}=\frac{d E_{t}}{d t}=-R i^{2}$ na Equação (2), Seção 5.21, e Equação (1d), Seção 5.22. $\therefore \frac{\mathrm{d}}{\mathrm{dV}} \mathrm{P}_{\mathrm{c}}=\rho \overrightarrow{\mathrm{v}} \cdot \overrightarrow{\mathrm{E}}$

Sabemos, pela lei de Ohm que a diferença de potencial: $\Delta V=R i$

Ré a resistência do circuito, onde passa uma corrente $i$ [ver Seção 5.21, Equação (1)]. ${ }^{65}$

$\Delta V=\int_{c} \vec{E} \cdot \overrightarrow{d \ell}$, ver lei de Faraday [Seção 5.14.1, Equação (1b)].

$$
P_{\mathrm{c}}=\int \rho \vec{v} \cdot \vec{E} d V=\left[\int \overrightarrow{\mathrm{E}} \cdot \mathrm{d} \ell\right] i=R i^{2} \quad(5.8 \mathrm{a} 2) \quad \mathrm{P}_{\mathrm{c}}=\Delta V i
$$

É potência dissipada em forma de calor por efeito Joule [ver Seção 5.21, $2^{\circ}$ termo da Equação (2), e Equação (1d) da Seção 5.22], pois:

$$
-d P_{c}=\rho \vec{v} \cdot \vec{E} d V=\frac{d q}{d V} \frac{\overrightarrow{d \ell}}{d t} \cdot \vec{E} d V=(\vec{E} \cdot \overrightarrow{d \ell}) \mathrm{i}
$$

Assim: $-\frac{d P_{c}}{d V}=\rho \vec{v} \cdot \vec{E}$, idem por unidade de volume.

Por exemplo, em um resistor, os elétrons livres que formam a corrente elétrica colidem com a rede cristalina. Essa transferência de energia acarreta um aumento de temperatura da rede. Em nosso caso, os elétrons e/ou íons se movimentam em algum meio material, ocasionando colisões em que há transferência da energia cinética das cargas móveis para o meio material e consequente dissipação na forma de energia térmica, ou seja, calor, devido ao aumento da amplitude das vibrações do meio. ${ }^{66}$

Note-se que considerar a diferença de potencial $\Delta V$ como $R i^{2}$, na condição de energia perdida, já foi considerada quando estudamos a energia do campo magnético na Seção 5.21, 2ํotermo da Equação (2).

A energia cinética deve ser acrescentada à energia total $\mathrm{u}_{\mathrm{t}}: \mathrm{u}_{\mathrm{t}}+\mathrm{u}_{\mathrm{cin}}=\sum \mathrm{u}$

${ }^{65}$ Um trecho de um circuito com características de resistência $\mathrm{R}$ provoca um $\Delta V=R i$ quando passa uma corrente $i$ pelo mesmo.

${ }^{66}$ Porém, de acordo com a Mecânica Quântica após Niels Bohr (ver Anexo 6, Equação 1a), quando um elétron livre se choca e retira um elétron de uma órbita periférica, outro elétron de uma órbita mais externa salta para ocupar seu lugar, gerando um quantum de energia cuja frequência pode ser conforme o grau de intensidade na faixa do infravermelho, que é energia térmica, frequência luminosa do vermelho ao azul, ou mesmo ultravioleta, que é ionizante. 
Agora, podemos fazer um balanço de energia no volume de controle considerado, levando em conta as diversas formas de energia existentes no $V C$. Analisando a variação no tempo, teremos, somando as Equações (5.7), (5.8) e (5.8a3):

$$
\frac{\partial}{\partial t}\left(\frac{1}{2} \varepsilon_{0} E^{2}+\frac{1}{2} \frac{B^{2}}{\mu_{0}}\right)+\rho \vec{v} \cdot \vec{E}
$$

Se chamarmos $\vec{H}=c \vec{B}$ de vetor campo magnético (LANDAU, BORISENKO): $\vec{B}=\frac{\vec{H}}{c}$

Ver Quadripotencial, Seção 5.25, Equação (4).

$$
\therefore B^{2}=\frac{H^{2}}{c^{2}} \quad \text { e } \quad c=\frac{1}{\sqrt{\varepsilon_{0} \mu_{0}}} \quad \therefore c^{2}=\frac{1}{\varepsilon_{0} \mu_{0}} \quad, \text { conforme Seção } 5.23 \text {, }
$$

Equação (5).

Em consequência: $\frac{1}{2} \varepsilon_{0} E^{2}+\frac{1}{2} \frac{H^{2}}{c^{2}} \frac{1}{\mu_{0}}=\frac{1}{2} \varepsilon_{0} E^{2}+\frac{1}{2} \frac{H^{2} \varepsilon_{0} \mu_{0}}{\mu_{0}}=\frac{1}{2} \varepsilon_{0} E^{2}+\frac{1}{2} \varepsilon_{0} H^{2}$

No sistema MKS, teremos: $\frac{\partial}{\partial t} \frac{1}{2} \varepsilon_{0}\left(E^{2}+H^{2}\right)+\rho \vec{v} \cdot \vec{E}$

Da Seção 5.25.2, após a Equação (5.5a), para se obter no sistema cgs, gaussiano: $\varepsilon_{0}=\frac{1}{4 \pi}$, logo: $\frac{\partial}{\partial t} \frac{E^{2}+H^{2}}{8 \pi}+\rho \vec{v} \cdot \vec{E}$ é potência por unidade de volume ou potência volumétrica.

A parcela: $W=\frac{E^{2}+H^{2}}{8 \pi}$ é denominada “densidade de energia do campo eletromagnético": energia por unidade de volume do campo, ou seja, energia volumétrica do campo.

O balanço de energia que consideramos se restringe à energia no interior do volume de controle. Isto é, a quantidade considerada se conserva.

Observemos que: $\frac{d}{d t} u_{c i n}=\rho \vec{v} \cdot \vec{E}$, conforme visto na Equação $(5.8 \mathrm{a} 1 \mathrm{~b})$, após a dedução de $\vec{f} \cdot \vec{v} d t$ (como vimos, $\overrightarrow{\mathrm{f}}_{\mathrm{m}} \cdot \overrightarrow{\mathrm{v}}=0 \therefore \overrightarrow{\mathrm{f}} \cdot \overrightarrow{\mathrm{v}}=\overrightarrow{\mathrm{f}}_{\mathrm{e}} \cdot \overrightarrow{\mathrm{v}}$ ).

Então: $\frac{E^{2}+H^{2}}{8 \pi}+u_{c i n}=c t e$

Em consequência: $\frac{d}{d t}\left(\frac{E^{2}+H^{2}}{8 \pi}+u_{\text {cin }}\right)=0$

Válido para sistema isolado. 


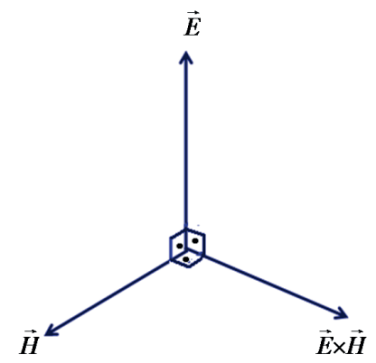

No entanto, em geral, o sistema não é isolado e pode receber ${ }^{67}$ energia do lado externo ao VC. Essa energia geralmente é por radiação, por intermédio de ondas eletromagnéticas. De acordo com o início da Secção 5.23, assinalado por (a), os vetores campo elétrico e magnético são perpendiculares entre si, por isso, seu produto vetorial: $\vec{E} \times \vec{H}$ é perpendicular a $\vec{E}$ e $\vec{H}$, sendo $\overrightarrow{\mathrm{H}}=\mathrm{c} \overrightarrow{\mathrm{B}}$, como visto na Equação (5.9). Esse vetor se propaga com a velocidade da luz $C$.

Da equação (5.8a1): $u_{B}=u_{e}=\frac{1}{2} \varepsilon_{0} E^{2} \therefore u_{t}=2 u_{e}=\varepsilon_{0} E^{2}$

$\mathrm{u}_{\mathrm{t}}$ é energia por unidade de volume, sendo c a velocidade da luz que é uma onda eletromagnética: $c=\frac{d e}{d t} \therefore d e=c d t$

de é o espaço percorrido pela onda no tempo $d t$.

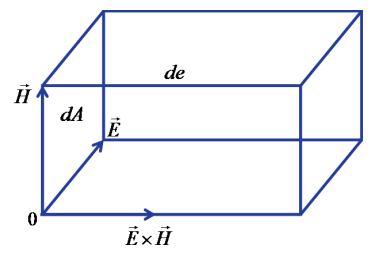

Sendo dA um elemento de área da frente de onda, o volume desenvolvido pela onda no intervalo de tempo dt será: $d A \cdot d e$. A quantidade de energia no volume é: $u_{t} d A \cdot c d t$.

Se dividirmos por $\mathrm{dA}$ e dt, teremos: $\mathrm{cu}_{\mathrm{t}}$, que é energia por unidade de área e por unidade de tempo.

Como vimos na Equação (5.9a), $u_{t}=\varepsilon_{0} E^{2}, \log o c u_{t}=c \varepsilon_{0} E^{2}(5.9 \mathrm{~b})$

Vimos na Equação (5.9) que $\vec{H}=c \vec{B}$, então $\vec{E} \times \vec{H}=c \vec{E} \times \vec{B}$

\footnotetext{
${ }^{67}$ Ou ceder para o exterior.
} 
Sabemos que da determinação da velocidade da luz, na Seção 5.23, Equação (9): $\frac{E}{B}=c$

Então, $c \vec{E} \times \vec{B}=c E \cdot \frac{E}{c}=E^{2}$, pois $\vec{E}$ e $\vec{B}$ são perpendiculares entre si, logo $\theta=90^{\circ}$ e sen $90^{\circ}=1$, também $\vec{E} \times \vec{H}=E^{2}$

Retomando $c \varepsilon_{0} E^{2}$ da Equação (5.9b), teremos: $\vec{P}=\frac{c}{4 \pi} \vec{E} \times \vec{H}(5.9 \mathrm{c})$

Pois $\varepsilon_{0}=\frac{1}{4 \pi}$. ver equação 5.5 a, seç̧ão 5.25. Não confundir com $\mathrm{P}_{\mathrm{c}}$, potência dissipada na forma de calor: $\mathrm{P}_{\mathrm{c}}=\mathrm{Ri}^{2}$, ver Equação (5.8a2).

O vetor $\vec{P}$ é denominado “vetor de Poynting”, em homenagem a John Henry Poynting (1852-1914), o primeiro a discutir as suas propriedades. A fórmula mencionada anteriormente está definida no sistema gaussiano. No sistema MKS, teremos: $\vec{P}=\frac{1}{\mu_{0}} \vec{E} \times \vec{B}$ em watt $/ \mathrm{m}^{2}(5.9 \mathrm{~d})$

Pois:

$\frac{c^{2}}{4 \pi} \vec{E} \times \vec{B}=\frac{\varepsilon_{0}}{\varepsilon_{0} \mu_{0}} \cdot \vec{E} \times \vec{B}$, sendo $c^{2}=\frac{1}{\varepsilon_{0} \mu_{0}}, \vec{H}=c \vec{B}$ e $\mu_{0}=4 \pi / c^{2}, \varepsilon_{0}=1 / 4 \pi$, como já vimos em:

Equação (5.9), Equação (5) da Seção 5.23 e Equação (5.5a) da Seção 5.25.

Da Seção 5.7, Equação (1), recordemos o divergente de uma função:

Sendo $\varphi_{\text {elm }}$ o fluxo de energia eletromagnética:

$$
\frac{d \varphi_{e l m}}{d V}=\lim _{\Delta V \rightarrow 0} \frac{\oiint_{S} \vec{P} \cdot \vec{n} d S}{\Delta V}=\frac{\partial P_{1}}{\partial x}+\frac{\partial P_{2}}{\partial y}+\frac{\partial P_{3}}{\partial z}=\operatorname{div} \vec{P}
$$

Em que $\vec{P}=P_{1} \vec{i}+P_{2} \vec{j}+P_{3} \vec{k}$

Ao calcularmos o divergente de uma função $\vec{P}$, transformamos a função $\vec{P}$ que é para área unitária, em outra função para volume unitário. Note que $\vec{P}$ era potência ${ }^{68}$ por área (por exemplo, $d A=d x d y$ ) se transformou em potência por área (dxdy) e por uma $3^{a}$ dimensão, $d z$, ou seja: por $d x d y$ cque é $d V$, volume elementar.

Calculando o div $\vec{P}$, transformamos em uma função compatível com a densidade de potência que é para volume unitário, ver Equação (5.9a1).

Podemos fazer um balanço de energia para um sistema de controle:

\footnotetext{
${ }^{68}$ Ou seja, potência, que é energia por unidade de tempo.
} 


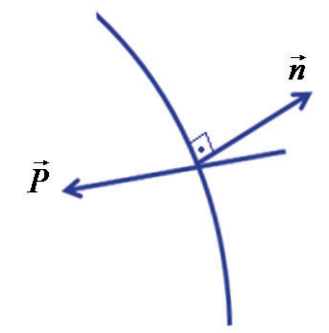

$$
\frac{\partial}{\partial t} \frac{E^{2}+H^{2}}{8 \pi}+\rho \vec{v} \cdot \vec{E}=-\operatorname{div} \vec{P}
$$

O sinal negativo significa que o vetor $\vec{P}^{69}$ penetra no sistema e forma um ângulo com a normal à superfície $\vec{n}$, maior que $90^{\circ}$ e, portanto, $\cos \alpha$ é negativo.

Essa equação é diferencial. Integrando-a, obtemos:

$$
\frac{\partial}{\partial t} \iiint_{V} \frac{E^{2}+H^{2}}{8 \pi} d V+\iiint_{V} \rho \vec{v} \cdot \vec{E} d V=-\iiint_{V} \operatorname{div} \overrightarrow{\mathrm{P}} \mathrm{d} v
$$

De fato, retornando a: $\frac{d \phi_{e l m}}{d V}=\vec{\nabla} \cdot \vec{P}=\frac{\partial \vec{P}_{i}}{\partial x_{i}}=\frac{\partial}{\partial x_{i}} P_{i}$, em notação tensorial, pela convenção de soma ou de Einstein:

$$
\begin{gathered}
\frac{\partial}{\partial x_{i}} P_{i}=\frac{\partial}{\partial x_{1}} P_{1}+\frac{\partial}{\partial x_{2}} P_{2}+\frac{\partial}{\partial x_{3}} P_{3} \\
d \phi_{e l m}=\vec{\nabla} \cdot \vec{P} d V, \text { integrando: } \quad \phi_{e l m}=\iiint_{V} \vec{\nabla} \cdot \vec{P} d V \quad \mathrm{~d} \phi_{\mathrm{elm}}=\left(\frac{\partial \mathrm{P}_{\mathrm{i}}}{\partial \mathrm{x}_{\mathrm{i}}} \mathrm{dx} \mathrm{x}_{\mathrm{i}}\right) \cdot \mathrm{dx}_{\mathrm{j}} \cdot \mathrm{dx}_{\mathrm{k}}
\end{gathered}
$$

Recordemos, da Seção 5.9, Equação (2) ao pé da página: $\frac{\partial}{\partial \mathrm{x}_{\mathrm{i}}} \int \mathrm{P}_{\mathrm{i}} \mathrm{dx}_{\mathrm{i}}=\mathrm{P}_{\mathrm{i}}$

Logo, ao usar na integral tripla, obteremos:

\footnotetext{
${ }^{69}$ Que vem de fora do sistema de controle. A origem do sistema de coordenadas está dentro do VC, por isso o vetor unitário $\vec{n}$ é dirigido para fora.
} 


$$
\phi_{\mathrm{e} \ell \mathrm{m}}=\iiint_{V} \vec{\nabla} \cdot \overrightarrow{\mathrm{P}} \mathrm{dV}=\iiint_{V} \frac{\partial \mathrm{P}_{\mathrm{i}}}{\partial \mathrm{x}_{\mathrm{i}}} \mathrm{dx}_{\mathrm{i}} \mathrm{dx}_{\mathrm{j}} \mathrm{dx}_{\mathrm{k}}=\iint_{S}\left[\int \frac{\partial \mathrm{P}_{\mathrm{i}}}{\partial \mathrm{x}_{\mathrm{i}}} \mathrm{dx}_{\mathrm{i}}\right] \mathrm{dx}_{\mathrm{j}} \mathrm{dx}_{\mathrm{k}}
$$

e: $\frac{\partial \mathrm{P}_{\mathrm{i}}}{\partial \mathrm{x}_{\mathrm{i}}} \mathrm{dx}_{\mathrm{i}}=\mathrm{dP}_{\mathrm{i}} \quad \int \mathrm{d} \mathrm{P}_{\mathrm{i}}=\mathrm{P}_{\mathrm{i}} \quad$ se torna integral dupla.

$\therefore \phi_{\text {elm }}=\iint_{S} \vec{P} \cdot d \vec{S}$, pois $\mathrm{dS}_{\mathrm{i}}=\mathrm{dx}_{\mathrm{j}} \mathrm{dx}_{\mathrm{k}}$ e $\mathrm{P}_{\mathrm{i}} \mathrm{dx}_{\mathrm{j}} \mathrm{dx}_{\mathrm{k}}=\overrightarrow{\mathrm{P}} \cdot \mathrm{d} \overrightarrow{\mathrm{S}}$ para $\mathrm{i}, \mathrm{j}, \mathrm{k}=1,2,3$

Pois i é perpendicular a j e a k.

$d V=d x_{i} \cdot d x_{j} \cdot d x_{k}$ da Seção $5.4, \quad \phi=\int_{S} \overrightarrow{\mathrm{v}} \cdot \overrightarrow{\mathrm{n}} \mathrm{dS} \quad$ e $\quad \mathrm{d} \overrightarrow{\mathrm{S}}=\overrightarrow{\mathrm{n}} \mathrm{d} S$

$\mathrm{d} \phi_{\mathrm{elm}}=\overrightarrow{\mathrm{P}} \cdot \mathrm{d} \overrightarrow{\mathrm{S}} \quad$ integrando:

$$
\phi_{\mathrm{elm}}=\iint_{\mathrm{S}} \overrightarrow{\mathrm{P}} \cdot \mathrm{d} \overrightarrow{\mathrm{S}} \quad \oiint_{S C} \vec{P} \cdot d \vec{S}=\iiint_{V C} \vec{\nabla} \cdot \vec{P} d V
$$

Obtemos

o teorema de Gauss-Ostrogradski, como vimos no final da Seção $5.8 .^{70}$

Usando esse teorema no balanço de energia, na forma integral, obtemos:

$$
\frac{\partial}{\partial t} \iiint_{V C} \frac{E^{2}+H^{2}}{8 \pi} d V+\iiint_{V C} \rho \vec{v} \cdot \vec{E} d V=-\oiint_{S C} \vec{P} \cdot \vec{n} d S
$$

Observemos que o fluxo de potência volta à sua forma original, isto é, como energia na unidade de tempo e na unidade de área, ver Equação (5.9d), $\overrightarrow{\mathrm{P}}$ no sistema MKS é em watt $/ \mathrm{m}^{2}$.

Qual é o significado dessa equação?

Ela estabelece que a taxa de variação da energia ${ }^{71}$ do campo eletromagnético dentro do $V C$, acrescida da variação da energia cinética devido ao trabalho executado pelo campo elétrico ao mover as cargas no $V C$, iguala o fluxo da energia eletromagnética que atravessa a $S C$, envolvendo o $V C$ na unidade de tempo. Deve-se notar que não há cargas na superfície do $V C$ nem cargas atravessando a superfície de controle $S C$. O vetor $\vec{P}$ representa a densidade do fluxo da energia no campo eletromagnético, atribuível ao fenômeno da radiação.

\footnotetext{
* A diferença entre a demonstração anterior e esta, é que a primeira foi feita para fluxo material e a segunda, para fluxo de energia. Porém, a equação de Einstein: $\mathrm{E}=\mathrm{c}^{2} \Delta \mathrm{m}$ [ver Seção 7.8, Equação (1)] mostra a equivalência entre massa e energia.

${ }^{71}$ De modo mais adequado, potência, que é energia por unidade de tempo.
} 
Essa equação pode ser deduzida de uma forma muito sucinta por intermédio do cálculo vetorial, no entanto, dessa forma perderíamos o entendimento mais profundo de cada termo da equação.

Tomando a equação diferencial, na Seção 5.15, Equação (2):

$\operatorname{rot} \vec{E}=-\frac{d \vec{B}}{d t}$ que é a lei de Faraday-Henry na forma diferencial, e lembrando que $\vec{B}=\frac{\vec{H}}{c}$ obtemos: $\frac{\partial \vec{H}}{\partial t}=-c \operatorname{rot} \vec{E}$

Multipliquemos escalarmente por $\vec{H}: \vec{H} \cdot \frac{\partial \vec{H}}{\partial t}=-c \vec{H} \cdot \operatorname{rot} \vec{E}$

A seguir, tomamos a lei de Ampère-Maxwell na forma diferencial, da Seção 5.16, Equação (5): $\operatorname{rot} \vec{B}=\mu_{0} \rho \vec{v}+\varepsilon_{0} \mu_{0} \frac{d \vec{E}}{d t}$, na forma gaussiana, conforme Seção 5.25, Equações (5.5a) e $(5.5 \mathrm{~b}): \mu_{0}=4 \pi / c^{2}$ e $\varepsilon_{0} \mu_{0}=\frac{1}{c^{2}}$ e $\overrightarrow{\mathrm{B}}=\overrightarrow{\mathrm{H}} / \mathrm{c}$ assim como da dedução da velocidade da luz $c$, Seção 5.23, Equação (5), $\frac{\mathrm{d} \overrightarrow{\mathrm{E}}}{\mathrm{dt}}=$ $\frac{1}{\varepsilon_{0} \mu_{0}} \operatorname{rot} \frac{\vec{H}}{c}-\frac{4 \pi}{c^{2}} \frac{1}{\varepsilon_{0} \mu_{0}} \rho \vec{v}=\frac{c^{2}}{c} \operatorname{rot} \overrightarrow{\mathrm{H}}-\frac{4 \pi}{c^{2}} c^{2} \rho \vec{v}$ obtemos: $\frac{\partial \vec{E}}{\partial t}=c \operatorname{rot} \overrightarrow{\mathrm{H}}-4 \pi \rho \overrightarrow{\mathrm{v}}$.

Multiplicando escalarmente por $\vec{E}: \vec{E} \cdot \frac{\partial \vec{E}}{\partial t}=c \vec{E} \cdot \operatorname{rot} \vec{H}-4 \pi \rho \vec{v} \cdot \vec{E}$

Adicionando os produtos escalares: $\vec{E} \cdot \frac{\partial \vec{E}}{\partial t}+\vec{H} \cdot \frac{\partial \vec{H}}{\partial t}=c \vec{E} \cdot \operatorname{rot} \vec{H}-4 \pi \rho \vec{V} \cdot \vec{E}-c \vec{H} \cdot \operatorname{rot} \vec{E}=$

$$
=c(\vec{E} \cdot \vec{\nabla} \times \vec{H}-\vec{H} \cdot \vec{\nabla} \times \vec{E})-4 \pi \rho \vec{V}
$$

Levando-se em conta que: $\quad \operatorname{div}(\overrightarrow{\mathrm{A}} \times \overrightarrow{\mathrm{B}})=\vec{\nabla} \cdot\left(\overrightarrow{\mathrm{A}}_{\mathrm{c}} \times \overrightarrow{\mathrm{B}}\right)+\vec{\nabla} \cdot\left(\overrightarrow{\mathrm{A}} \times \overrightarrow{\mathrm{B}}_{\mathrm{c}}\right)^{72}$

Como visto na Seção 5.26, Equação (5.8a1a), o triplo produto escalar pode ser expresso por um determinante:

$$
\vec{\nabla} \cdot\left(\vec{A}_{c} \times \vec{B}\right)=\left|\begin{array}{ccc}
\partial / \partial x_{1} & \partial / \partial x_{2} & \partial / \partial x_{3} \\
A_{c_{1}} & A_{c_{2}} & A_{c_{3}} \\
B_{1} & B_{2} & B_{3}
\end{array}\right|
$$

E, da mesma forma, $\vec{\nabla} \cdot\left(\vec{A} \times \vec{B}_{c}\right)$

Esse produto é invariante nas permutações cíclicas dos vetores, isto é, quando as linhas do determinante mudam de posição ciclicamente, mas mudam de sinal se a permutação não for cíclica.

Em consequência: $\operatorname{div}(\vec{A} \times \vec{B})=-\vec{A}_{c} \cdot(\vec{\nabla} \times \vec{B})+\vec{B}_{c} \cdot(\vec{\nabla} \times \vec{A})=\vec{B} \cdot \operatorname{rot} \vec{A}-\vec{A} \cdot \operatorname{rot} \vec{B}$

2 - $\nabla$ Age separadamente em cada fator, deixando o outro constante (daí o índice $\mathcal{C}$ ), como uma derivada, aliás é uma derivada espacial. 
Comparando com a equação $5.9 \mathrm{f}$ e aplicando à soma dos produtos escalares:

$$
\begin{aligned}
& \vec{E} \cdot \frac{\partial \vec{E}}{\partial t}+\vec{H} \cdot \frac{\partial \vec{H}}{\partial t}=c(\vec{E} \cdot \vec{\nabla} \times \vec{H}-\vec{H} \cdot \vec{\nabla} \times \vec{E})-4 \pi \rho \vec{V}= \\
& =-c(\vec{\nabla} \cdot \vec{E} \times \vec{H}-\vec{\nabla} \cdot \vec{H} \times \vec{E})-4 \pi \rho \vec{V}
\end{aligned}
$$

E aplicando à soma dos produtos escalares:

$$
\frac{1}{2} \frac{\partial}{\partial t} \frac{E^{2}+H^{2}}{4 \pi}=-\operatorname{div}\left[\frac{c}{4 \pi}(\vec{E} \times \vec{H})\right]-\rho \vec{v} \cdot \vec{E} \text {, pois } \frac{\partial}{\partial t} E^{2}=2 \vec{E} \cdot \frac{\partial \vec{E}}{\partial t} \quad E^{2}=\vec{E} \cdot \vec{E}
$$

Em que: $\vec{P}=\frac{c}{4 \pi}(\vec{E} \times \vec{H})$ é o vetor de Poynting como já visto na Equação (5.9c).

Então: $\frac{\partial}{\partial t} \frac{E^{2}+H^{2}}{8 \pi}+\rho \vec{v} \cdot \vec{E}=-\operatorname{div} \vec{P}$

É o balanço de energia em forma diferencial obtido na Equação (5.9e).

Como a primeira dedução do balanço de energia ficou extensa, vamos resumi-la: consideremos um $V C$ vazio, isto é, sem cargas elétricas, onde transitam os campos elétrico e magnético associados. Em consequência desses campos, teremos as energias volumétricas, ou seja, energias por unidade de volume.

Elétrica: $u_{e}=\frac{1}{2} \varepsilon_{0} E^{2}$

$$
u_{e}=u_{B}=\frac{1}{2} \varepsilon_{0} E^{2} \text {, ver Equação (5.8a1). }
$$

Magnética: $u_{B}=\frac{1}{2} \frac{B^{2}}{\mu_{0}}=\frac{1}{2} \varepsilon_{0} E^{2}=\frac{1}{2} \varepsilon_{0} H^{2}$, ver equação $5.8 \mathrm{a} 1,5.9$ e seguintes

A soma será: $u_{t}=\varepsilon_{0} E^{2}$. Vamos denominá-la "densidade de energia do campo eletromagnético", ou seja, energia volumétrica do campo, no sistema MKS: $W=\frac{1}{2} \varepsilon_{0}\left(E^{2}+H^{2}\right)$ e no sistema cgs ou gaussiano: $W=\frac{E^{2}+H^{2}}{8 \pi} \quad \varepsilon_{0}=\frac{1}{4 \pi}$

Se houver cargas elétricas e um meio material, essas cargas ficarão sujeitas, por unidade de volume, à força volumétrica de Lorentz: $\vec{f}=\rho \vec{E}+\rho \vec{v} \times \vec{B}$. As cargas se deslocarão de uma distância $d \vec{r}$ no tempo $d t$, ganhando a energia cinética: $\vec{f} \cdot d \vec{r}=\vec{f} \cdot \vec{v} d t$.

Verifica-se então que, das duas parcelas de $\vec{f}$, a elétrica $\rho \vec{E}$ e a magnética $\rho \vec{v} \times \vec{B}$, a única que contribuirá para a variação da energia cinética será a elétrica, pois a magnética, sendo: $\rho \vec{v} \times \vec{B} \cdot \vec{v} d t$, com dois vetores iguais, $\vec{v}$, se anula [ver Equação (5.8a1a)]. 
Em consequência, a variação volumétrica da energia cinética, será: $d u_{c i n}=\rho \vec{v} \cdot \vec{E} d t$, e a variação na unidade de tempo: $\frac{d}{d t} u_{\text {cin }}=\rho \vec{v} \cdot \vec{E}$.

Essa energia causa colisões das cargas móveis com o meio material, com o consequente aumento da amplitude de vibração do meio, resultando na dissipação da energia cinética na forma de energia térmica. Esse efeito é denominado efeito Joule, tendo como expressão, conforme a lei de Ohm [ver Equação (5.8a2)]: $P_{c}=R_{i}^{2}$, potência dissipada. Na unidade de volume: $\frac{d P_{c}}{d V}=\rho \vec{V} \cdot \vec{E}$, ver Equação (5.8a3).

No interior do $V C$ teremos uma variação de energia volumétrica:

$$
\frac{\partial}{\partial t} \frac{E^{2}+H^{2}}{8 \pi}+\rho \vec{v} \cdot \vec{E}
$$

Se esse sistema for conservativo, a expressão anterior se anulará e: $\frac{E^{2}+H^{2}}{8 \pi}+u_{c i n}=c t e$, válido para sistema isolado.

Contudo, em geral, o sistema não é isolado e pode receber ou ceder energia para o exterior. Essa energia é o que denominamos de vetor de Poynting, e se manifesta como radiação que atravessa a $S C$ do $V C$, potência por unidade de superfície: $\vec{P}=\frac{c}{4 \pi} \vec{E} \times \vec{H}$ no sistema cgs [ver Equação (5.9c)]. Não confundir com $\mathrm{P}_{\mathrm{c}}=\mathrm{Ri}^{2}$, potência dissipada na forma de calor.

O balanço de energia será:

$$
\begin{gathered}
\frac{\partial}{\partial t} \frac{E^{2}+H^{2}}{8 \pi}+\rho \vec{v} \cdot \vec{E}=-\operatorname{div} \vec{P}, \text { em forma integral: } \\
\frac{\partial}{\partial t} \iint_{V C} \frac{E^{2}+H^{2}}{8 \pi} d V+\iiint_{V C} \rho \vec{v} \cdot \vec{E} d V=-\iiint_{V C} \vec{\nabla} \cdot \vec{P} d V=-\iint_{S C} \vec{P} \cdot \vec{n} d S
\end{gathered}
$$

